\title{
中国半翅目等29目昆虫2020年新分类单元
}

\author{
刘童神 1 1,2, 陈静 ${ }^{1}$, 姜立云 ${ }^{1}$, 乔格侠 (1) 1 2*
}

1. 中国科学院动物研究所动物进化与系统学重点实验室, 北京 100101; 2. 中国科学院大学生命科学学院, 北京 100049

摘要: 以中国半翅目等29目昆虫类群(除鞘翅目、双翅目、鳞翅目、膜翅目之外的其他目)为研究对象, 基于2020年度发表的 新分类单元与文献资料, 编制了物种名录, 分析了新分类单元的地理分布与模式标本存放情况等。2020年, 中国半翅目等29 目昆虫共描述349个新分类单元，包括新属35个，新种311个，新亚种3个; 新种发表的热点地区在我国的西南山地及其邻近地 区。模式标本存放单位多达69家, 其中正模标本主要存放在国内保藏机构, 而境外机构保藏的标本较少且以副模标本为主。 本年度描述的349个新分类单元由356位作者所贡献, 共发表文献182篇; Zootaxa和ZooKeys 是发表的主要期刊。本次调查为进 一步深入研究物种演化的时空格局及生物多样性保护提供了基础数据。同时, 由于人类面临生物多样性急剧下降的威胁, 应 加快大数据平台的建设及其多样化信息的挖掘, 支撑生物多样性的研究和保护。

关键词: 生物多样性信息学; 半翅目; 直翅目; 新分类单元; 中国

刘童祎, 陈静, 姜立云, 乔格侠 (2021) 中国半翅目等29目昆虫2020年新分类单元. 生物多样性, 29, 1050-1057. doi: 10.17520/biods.2021200.

Liu TY, Chen J, Jiang LY, Qiao GX (2021) Annual report of new taxa for Chinese Hemiptera and 28 other orders of Insecta in 2020. Biodiversity Science, 29, 1050-1057. doi: 10.17520/biods.2021200.

\section{Annual report of new taxa for Chinese Hemiptera and 28 other orders of Insecta in 2020}

Tongyi Liu ${ }^{(1,2}$, Jing Chen ${ }^{1}$, Liyun Jiang ${ }^{1}$, Gexia Qiao ${ }^{\circledR 1,2^{*}}$

1 Key Laboratory of Zoological Systematics and Evolution, Institute of Zoology, Chinese Academy of Sciences, Beijing 100101

2 College of Life Sciences, University of Chinese Academy of Sciences, Beijing 100049

\section{ABSTRACT}

Aims: This study aims to summarize the new taxa of Chinese Hemiptera and 28 other orders of Insecta (i.e., orders other than Coleoptera, Diptera, Lepidoptera, and Hymenoptera) in 2020.

Methods: We collected information about newly described taxa, including new genera, species, and subspecies for Chinese Hemiptera and 28 other insect orders in 2020 by searching journals and the Zoological Record database. Based on the described taxa and published literature, we compiled a catalogue of type specimens and a bibliography for new species of Chinese Hemiptera and other insects described in 2020. We also analyzed the biodiversity information provided by type specimens, such as authors, locations, and preservation sites.

Results: In 2020, a total of 349 new taxa, including 35 new genera, 311 new species, and 3 new subspecies, were described in Chinese Hemiptera and 28 other insect orders. The hotspots for the new species described were in Southwest China and the adjacent areas. Additionally, there are as many as 69 institutions that are designated for the deposition of type specimens; within these, the holotype was primarily collected in domestic collection institutions. In contrast, there were relatively few type specimens collected by foreign institutions, and those collected were mainly paratypes. The 349 new taxa described this year were contributed by 356 authors, and a total of 182 articles were published. Zootaxa and ZooKeys were the main publication journals for these descriptions.

Conclusion: This study provides baseline data for further in-depth studies in the temporal and spatial patterns of species evolution and biodiversity protection in China. Moreover, in the face of today's rapidly declining biodiversity, we should accelerate the construction of data platforms and the integration of diverse information sources to strengthen the biodiversity research and protection.

收稿日期: 2021-05-19; 接受日期: 2021-08-09

基金项目: 中国科学院战略生物资源专项(KFJ-BRP-003)和国家科技资源共享服务平台国家动物标本资源库2021年度运行费

* 通讯作者 Author for correspondence. E-mail: qiaogx@ioz.ac.cn 
Key words: biodiversity informatics; Hemiptera; Orthoptera; new taxa; China

标本采集和物种描述是认识和记录地球生物 多样性的重要途径之一。生物标本往往是有机体形 态、生态以及遗传多样性等特征的载体, 并同时兼 有时间(采集时间)、空间 (采集地点)以及性状等三大 属性数据, 具有重要的科研价值(Andrew et al, 2019)。因此, 生物标本的采集、描述以及保存可以 极大地推进以生物分类学为基础的众多研究领域 的蓬勃发展, 如宏进化、宏生态以及保护生物学等 (Nelson \& Ellis, 2019)。近年来, 随着生物多样性信 息学的快速发展, 越来越多的标本资源被数字化, 并可通过数据共享平台公开获取, 这极大地促进了 物种的发现、描述以及生物多样性研究等领域的发 展(王昕等, 2017)。然而, 在拥有海量数据的大型生 物多样性数据库中, 中国生物多样性的信息并不 多。以全球生物多样性信息网络(Global Biodiversity Information Facility, GBIF; http://www.gbif.org/)为例, 截止到2021年3月 31 日, 已涵盖16亿条分布数据, 而中国仅有 0.5 亿条, 这在一定程度上限制了人们 对中国生物多样性的了解。

中国作为全球生物多样性最丰富的国家之一, 拥有 37,793种植物、54,359种动物和12,506种真菌 (The Biodiversity Committee of Chinese Academy of Sciences, 2020), 并有较高的植物和脊椎动物特有 性。在 36 个全球生物多样性热点地区中 (https://www.conservation.org/priorities/biodiversityhotspots), 有4个全部或者部分分布在中国(中国西 南山区、喜马拉雅山脉地区、印度-缅甸地区以及 中亚山区)。此外, 青藏高原隆升及其引起的环境异 质性和东亚季风的形成(Molnar et al, 2010), 为东亚 的物种分化及其在剧烈的气候波动中维持多样性 提供了有力的保障(Xing \& Ree, 2017)。与同纬度的 欧洲和北美洲地区相比, 中国拥有最多样化的生物 类群(Qian et al, 2017)。现在中国生物标本馆(博物馆) 的标本藏量不断扩大(贺鹏等, 2019), 生物多样性研 究也不断取得新的突破(Mi et al, 2021)。

昆虫是地球上生物多样性的重要组成部分, 无 论遗传多样性、物种多样性、性状和功能多样性都 是最丰富的生物类群。然而, 对于昆虫的研究宽度 与深度, 与高等植物、脊椎动物等类群相比都明显
落后。据估计全球约有昆虫 550 万种(Stork et al, 2015), 而已定名和描述的仅有约 100 万种，近 $80 \%$ 的昆虫物种迄今尚未被认知或记录(Stork, 2018)。对 于生物资源极为丰富的中国而言, 自新中国成立以 来, 昆虫分类学研究的发文量由早期的每年几十篇 到如今的每年至少 500 篇, 每年发表新种数量也翻 了20多倍(图1)。这不仅得益于我国拥有一支强大的 分类学研究队伍, 还得益于国家对基础研究工作的 大力支持。但是, 值得注意的是, 自2010年以后, 每 年发表的分类学文章以及新分类单元的数量均呈 现缓慢下降的趋势(图1)。这一现象对存在许多尚未 被发现和描述的昆虫类群及其学科的发展来说是 不利的。随着全球人口的激增, 伴随而来的高强度 的人类活动对自然生态系统带来了巨大影响 (Newbold et al, 2015), 而人类世的人为活动也对昆 虫的多度、生物量、物种多样性以及分布区大小都 带来了极大的负面效应(Wagner et al, 2021)。近几十 年, 我国生物多样性也受到严重的威胁(魏辅文等, 2014)。考虑到目前人为介导的高物种灭绝率 (Wagner et al, 2021)以及许多尚未被记录的物种及 其栖息地可能在被发现之前就已经消失(Barnosky et al, 2011), 我们需要在中国开展与生物调查和物 种描述与定名工作相关的物种年度总结, 以进一步 推动数据共享平台的建设, 并为评估人类活动对生 物多样性的影响奠定基础。

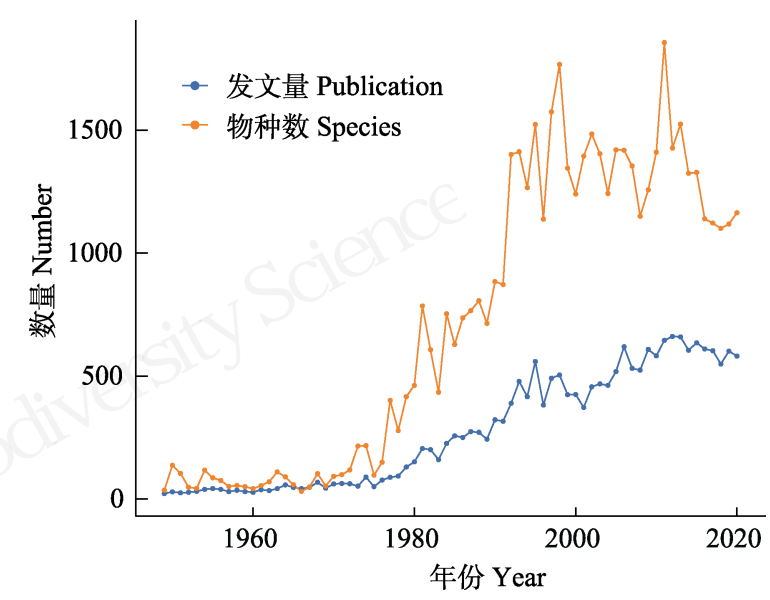

图1 1949年以来中国昆虫年度发表文章数量及新种数量 Fig. 1 Annual numbers of publication and new species for Chinese insects since 1949 
表1 本研究关注的类群及其2020年度发表新类群的情况 Table 1 The taxa involved in this research and the basic status of new taxa published in 2020

\begin{tabular}{|c|c|c|c|}
\hline $\begin{array}{l}\text { 目 } \\
\text { Order }\end{array}$ & $\begin{array}{l}\text { 新属 } \\
\text { New genus }\end{array}$ & $\begin{array}{l}\text { 新种及新亚种 } \\
\text { New species and } \\
\text { subspecies }\end{array}$ & $\begin{array}{l}\text { 总计 } \\
\text { Total }\end{array}$ \\
\hline 半翅目 Hemiptera & 16 & 91 & 107 \\
\hline 直翅目 Orthoptera & 12 & 76 & 88 \\
\hline 襀翅目 Plecoptera & 3 & 44 & 47 \\
\hline 毛翅目 Trichoptera & 0 & 15 & 15 \\
\hline 蜚蠊目 Blattodea & 0 & 14 & 14 \\
\hline 䗛目 Phasmatodea & 1 & 12 & 13 \\
\hline 䖝目 Phthiraptera & 2 & 10 & 12 \\
\hline 弹尾目 Collembola & 1 & 10 & 11 \\
\hline 纱翅目 Thysanoptera & 0 & 10 & 10 \\
\hline 长翅目 Mecoptera & 0 & 9 & 9 \\
\hline 脉翅目 Neuroptera & 0 & 8 & 8 \\
\hline 蜉蝣目 Ephemeroptera & 0 & 4 & 4 \\
\hline 螳螂目 Mantodea & 0 & 4 & 4 \\
\hline 蜻蜓目 Odonata & 0 & 3 & 3 \\
\hline 原尾目 Protura & 0 & 2 & 2 \\
\hline 革翅目 Dermaptera & 0 & 1 & 1 \\
\hline 蚤目 Siphonaptera & 0 & 1 & 1 \\
\hline 双尾目 Diplura & 0 & 0 & 0 \\
\hline 石蛃目 Archaeognatha & 0 & 0 & 0 \\
\hline 衣鱼目 Zygentoma & 0 & 0 & 0 \\
\hline 等翅目 Isoptera & 0 & 0 & 0 \\
\hline 蛩蠊目 Grylloblattodea & 0 & 0 & 0 \\
\hline 螳蝉目 Mantophasmatodea & 0 & 0 & 0 \\
\hline 纺足目 Embioptera & 0 & 0 & 0 \\
\hline 缺翅目 Zoraptera & 0 & 0 & 0 \\
\hline 啮虫目 Psocoptera & 0 & 0 & 0 \\
\hline 广翅目 Megaloptera & 0 & 0 & 0 \\
\hline 蛇蛉目 Raphidioptera & 0 & 0 & 0 \\
\hline 捻翅目 Strepsiptera & 0 & 0 & 0 \\
\hline 总计 Total & 35 & 314 & 349 \\
\hline
\end{tabular}

本研究以半翅目等 29 目昆虫类群(除鞘翅目、双 翅目、鳞翅目、膜翅目等昆虫纲四大目之外的类群) 为研究对象 (表1), 以隐藏在模式标本中重要的生物 多样性信息(如命名人、采集地点等)为基础, 总结 2020年度我国半翅目等 29 目昆虫新分类单元的描 述与发表情况, 编制名录, 评估物种组成并对标本 存放数据进行初步分析, 从而揭示我国半翅目等 29目昆虫的物种多样性、生物资源调查与研究的广 度和深度, 并在一定程度上反映昆虫分类学的发 展趋势。

\section{材料与方法}

基于世界上历史最悠久且至今仍在不断更新 的动物学记录数据库(Zoological Record, ZR), 系统 检索和编汇2020年度发表的中国半翅目等29目昆 虫的物种名称，数据统计时间截止到2021年3月 1 日。鉴于ZR数据库对国内刊物的收录稍微滞后的现 状，本研究对2020年度发表在《昆虫分类学报》《动 物分类学报》等国内期刊上的新分类单元进行了单 独检索。通过筛选和整理, 共获得2020年度半翅目 等昆虫新分类单元名称 630 条, 其中每条数据包含 物种分类阶元、命名人、标本采集信息(数量、正模、 副模、采集地理位置、保藏单位等), 以及文献发表 时间和期刊信息等。对于缺少经纬度的采集信息， 使用Google Earth进行搜索; 基于 $200 \mathrm{~km} \times 200 \mathrm{~km}$ 的栅格对该年度发表新种分布的热点地区进行可 视化。同时，为反映1990-2020年整个昆虫纲发表新 种的地理分布趋势，以中国34个省级行政区为地理 操作单元，对31年整个昆虫纲每年发表新种的分布 数据进行了整理(注: 1990年以前, ZR数据库中地理 分布信息常以China标注，而没有细分到34个省级 行政区，故只进行了该期间每年发表新种的统计)。

\section{2 结果与分析}

\subsection{0 年度发表新分类单元的基本状况}

经统计，2020年度中国半翅目等29目昆虫共描 述349个新分类单元，包括新属 35 个, 新种 311 个, 新亚种 3 个(附录1)。在所统计的 29 目昆虫类群中，描 述的新分类单元主要集中在半翅目、直翅目和襀翅 目，分别描述了 107 个、 88 个和 48 个新分类单元，分 别占总数的 $31 \% 、 25 \%$ 和 $14 \%($ 表 1$)$ 。而有 12 个目我 国在2020年度没有发表新分类单元，主要是一些较 为原始且中国记录种类少的类群，如石蛃目、衣鱼 目和纺足目等。

发表的 35 个新属隶属于 6 目 16 科。描述新属最 多的为半翅目和直翅目, 其次为風目和襀翅目, 弹 尾目和䗛目分别描述了1个新属。其中, 11 个属的描 述和建立是基于详实的形态特征和分子数据。

发表的 314 个新种和新亚种隶属于 76 科和 183 属。以科为单位统计发现, 新种描述最多的科是半 翅目叶蝉科, 共描述38个新种及新亚种(表2)。由于 该类群为植食性昆虫, 危害多种经济作物, 而且国 
内有多个团队从事该科的系统分类, 因此该类群得 到更多的关注。以属为单位统计发现, 描述新种和 新亚种最多的为直翅目的裸灶螯属(Tachycines), 共 发表了12个新种。

在2020年度发表新种的研究中, 大多数是基于 传统形态学研究, 从整合分类(如分子、生态以及形 态等多证据)的角度对某个类群系统厘定的研究较 少。其中，仅有24个新种在发表时提供了分子序列。 在这些物种发表时，常用的分子标记为线粒体基因 $C O I 、 C y t b 、 18 S$ rRNA、28S rRNA, 以及核基因18S rDNA和28S rDNA。其中, $\mathrm{Li}$ 等(2020)提供了完整的 线粒体基因组数据。基于分子数据的物种界定分析 中, 常用的方法为基于单位点的界定方法, 如 ABGD (Automatic Barcode Gap Discovery)、GMYC (General Mixed Yule Coalescent)等; 而相对更加稳 健的基于多物种溯祖理论的 BPP (Bayesian Phylogenetics and Phylogeography programme)方法 在昆虫物种界定的研究中应用较少。近年来, 基于 整合框架的物种界定越来越受到关注(Padial et al,
2010; Fujita et al, 2012)。因此, 分类学家应该给予整 合分类更多的重视以应对未来的挑战。

\section{2 中国新物种的地理分布}

统计1990-2020年整个昆虫纲每年发表的新物 种发现, 新种主要分布在在我国的西南(如云南、四 川、贵州等)及其邻近地区(如西藏、广西和陕西等) (图2a)。同时，台湾岛和海南岛等岛屿，以及浙江、 福建和广东等东南沿海地区也发现了较多的新物 种。其中，云南发表的新种多达7,688种，其次是四 川(5,767种)、台湾(4,117种)、贵州(1,953种)和西藏 $(1,897$ 种)。而华北地区在这31年间发表新种的数量 较少, 如河北和山东分别描述了 217 个和 122 个新 种。总的来说, 物种发表数量从西南到东北呈现减 少趋势。

2020年度发表的半翅目等29目昆虫的新种主 要分布在云南、广西、贵州、海南和四川(图2b)。 西南山地是发现新物种最多的区域，其中云南发现 了101个新种和3个新亚种，占2020年度 29 目昆虫新 种数量的 $1 / 3$; 贵州和四川分别发表了 33 个和 22 个

表2 2020年发现5个以上新物种的科和属

Table 2 The families and genera that discovered more than five new species in 2020

\begin{tabular}{|c|c|c|c|c|c|}
\hline $\begin{array}{l}\text { 科 } \\
\text { Family }\end{array}$ & $\begin{array}{l}\text { 新属 } \\
\text { New genus }\end{array}$ & $\begin{array}{l}\text { 新种及新亚种 } \\
\text { New species and subspecies }\end{array}$ & $\begin{array}{l}\text { 总计 } \\
\text { Total }\end{array}$ & $\begin{array}{l}\text { 属 } \\
\text { Genus }\end{array}$ & $\begin{array}{l}\text { 新种及新亚种 } \\
\text { New species and subspecies }\end{array}$ \\
\hline 叶蝉科 Cicadellidae & 6 & 38 & 44 & 疾灶蚉属 Tachycines & 12 \\
\hline 蚉斯科 Tettigoniidae & 5 & 26 & 31 & 禇翅蠊属 Anaplecta & 8 \\
\hline 襀科 Perlidae & 0 & 19 & 19 & 倍叉襀属 Amphinemura & 7 \\
\hline 叉襀科 Nemouridae & 1 & 16 & 17 & 拟大磨蚱属 Macromotettixoides & \\
\hline 驼螽科 Rhaphidophoridae & 0 & 16 & 16 & 纺织娘属 Mecopoda & 7 \\
\hline 蚱科 Tetrigidae & 1 & 14 & 15 & 刺异䗛属 Oxyartes & 6 \\
\hline 漂蜡蝉科 Issidae & 4 & 9 & 13 & 塔氏小叶蝉 Takagioma & 6 \\
\hline 笛䗛科 Diapheromeridae & 0 & 10 & 10 & 叶蛾蜡蝉属 Atracis & 5 \\
\hline 褶翅蠊科 Anaplectidae & 0 & 8 & 8 & 多突石蛾属 Ceraclea & 5 \\
\hline 扁蝽科 Aradidae & 3 & 5 & 8 & 零叶蝉属 Limassolla & 5 \\
\hline 蛉蟋科 Trigonidiidae & 4 & 4 & 8 & 束长蝽属 Malcus & 5 \\
\hline 长角鸟虫科 Philopteridae & 2 & 6 & 8 & & \\
\hline 网襀科 Perlodidae & 2 & 6 & 8 & & \\
\hline 菱蜡蝉科 Cixiidae & 0 & 7 & 7 & & \\
\hline 长角石蛾科 Leptoceridae & 0 & 7 & 7 & & \\
\hline 管蓟马科 Phlaeothripidae & 0 & 6 & 6 & & \\
\hline 姬蠊科 Ectobiidae & 0 & 5 & 5 & & \\
\hline 蛾蜡蝉科 Flatidae & 0 & 5 & 5 & & \\
\hline 束长蝽科 Malcidae & 0 & 5 & 5 & & \\
\hline 蝎蛉科 Panorpidae & 0 & 5 & 5 & & \\
\hline
\end{tabular}



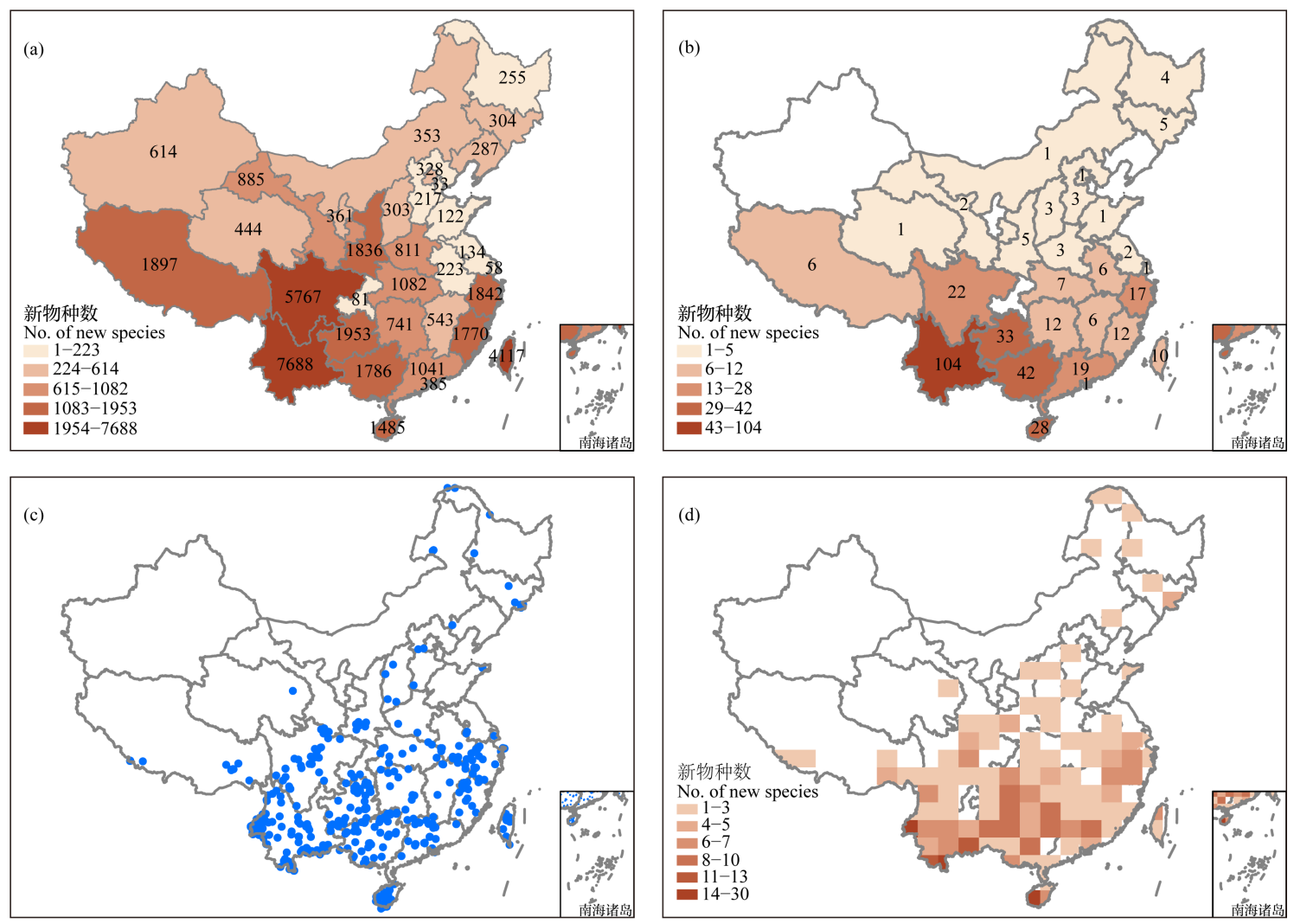

图2 中国昆虫新物种的地理分布。(a) 1990-2020年中国各省发现的昆虫新物种数量; (b) 2020年度半翅目等29个目各省发 现的物种数量; (c) 2020年度半翅目等29个目新物种分布点; (d) 2020年度半翅目等 29 个目新物种在 $200 \mathrm{~km} \times 200 \mathrm{~km}$ 的分布 格局。

Fig. 2 Geographical distribution of new insect species in China. (a) The numbers of new insect species discovered in various provinces of China from 1990 to 2020; (b) The numbers of species discovered in each province for Hemiptera and 28 other orders of Insecta in 2020; (c) The occurences of new species of Hemiptera and 28 other orders of Insecta in 2020; (d) Distribution pattern of new species of Hemiptera and 28 other orders of Insecta under the $200 \mathrm{~km} \times 200 \mathrm{~km}$ grid cells.

新种。其次, 我国南方沿海地区也是新物种发现的 主要区域，广西、海南和广东分别发表42个、28个 和19个新种。基于 $200 \mathrm{~km} \times 200 \mathrm{~km}$ 的栅格尺度, 2020年度中国半翅目等29目昆虫新种发表的热点 地区为云南西部的中缅边境和南部的西双版纳地 区(图2c, d)。

无论是基于 31年整个昆虫纲新种地理分布还 是2020年度物种发表结果, 西南山地都是昆虫新种 发现的热点地区之一(图2)。中国西南山地是全球36 个生物多样性保护热点地区之一, 西起藏东南, 向 北延伸至川西地区, 横亘云南中北部。该地区具有 独特的异质性地形和复杂的区系历史, 是东亚地区 物种多样性的“物种䈋” (species pump)和/或“物种 博物馆” (species museum) (雷富民等, 2021), 为从 宏进化和宏生态角度探讨成种机制提供了有利条 件。然而该地区的生物多样性数据仍十分缺乏( Mi et al, 2021), 尤其是该地区昆虫的相关研究十分薄 弱。尽管本研究仅基于昆虫新种发表的数据, 但无 论是基于单年份还是跨越30余年时间尺度的数据, 均发现该地区具有非常高的昆虫物种多样性。因此, 未来应加强在西南山地以昆虫为研究对象, 从不同 的时空尺度来探讨物种起源和演化问题的工作。另 外, 值得注意的是, 与高等植物和脊椎动物相比, 昆虫缺乏物种的保护等级评估, 从而影响了生物多 样性热点地区保护优先权的制定。因此, 我们应加 强对昆虫物种的保护等级评估, 并在今后制定保护 计划和管理时给予昆虫类群更多的关注。

\section{3 模式标本存放情况}

博物馆标本往往可以同时体现时间、空间以及 性状等3个方面的属性。因此在生物多样性、全球 气候变化以及环境健康等研究领域发挥着重要作 用(贺鹏等, 2021)。而模式标本在包含博物馆标本的 
自然属性和应用之外, 其馆藏量还反映了一个国家 或地区分类学研究的水平和关注度。

2020年度发表的中国半翅目等29目昆虫模式 标本存放地比较分散, 多达69处(附录2)。主要保藏 机构包括39家高校、11个科研院所和11个博物馆, 以及 4 个个人保藏地和 4 个其他类型的保藏地。从保 藏标本的类型来看, 正模标本主要存放在国内的保 藏机构, 境外机构保藏的标本较少且以副模标本为 主。其中, 模式标本保藏数量前三位的单位均为高 校, 即西北农林科技大学、贵州大学和河北大学, 分别保藏了 43 种、32种和 30 种。在这3所高校中, 保 藏的模式标本类群有所不同, 体现了这些高校的研 究团队分别关注不同的研究类群。其中, 西北农林科 技大学保藏的主要为半翅目和长翅目, 以及少数的 螳螂目模式标本; 贵州大学保藏的主要为半翅目和 直翅目; 而河北大学则以直翅目为主。模式标本保 藏较多的科研单位以中国科学院为主, 其中中国科 学院动物研究所有 16 种, 中国科学院上海昆虫博物 馆有 12 种。私人保藏的标本较少, 总计 6 种, 且以国 内藏家为主。

以类群划分来看, 半翅目模式标本的保藏单位 涉及23个, 以西北农林科技大学和贵州大学保藏的 模式标本最多, 分别为34种和28种。76种直翅目模 式标本共涉及 18 个保藏单位, 其中河北大学保藏了 30 种，占 $39 \%$; 其次是中国科学院动物研究所有 9 种。而襀翅目模式标本的保藏单位主要在河南科技
学院、中国农业大学和江苏科技大学, 分别保藏了 20 种、 10 种和 9 种。其他类群模式标本存放见附录 2 。

\section{4 发表新分类单元的学者和研究单位}

2020年，共有356个作者参与了中国半翅目等 29 目昆虫的 349 个新分类单元的描述与发表。其中 中国学者294位, 外籍学者62位。参与发表 10 个以上 新种的学者有 11 人(表3), 他们主要来自国内不同的 高校。参与发表新种最多的学者是西北农林科技大 学的张雅林(Yalin Zhang), 本年度共描述和发表了 33 个新种, 他主要关注半翅目昆虫, 同时也涉及了 螳螂目的物种。此外, 参与发表了2-4个新物种的学 者有78位; 仅参与发表1个新类群的作者占 $74 \%$ 。

\section{5 发表新种的刊物}

2020年，发表的中国半翅目等29目昆虫349个 新分类单元共涉及 182 篇论文以及 16 个国际与国内 期刊(附录1)。其中约91\%的文章发表在Zootaxa、

ZooKeys、《昆虫分类学报》以及European Journal of Taxonomy 4种期刊上(图3), 剩下12本期刊则发表了 其余9\%的相关文献。2020年度共171篇文献(占总发 文量的92\%)发表在科学引文索引(SCI; Anonymous, 2021)收录的刊物上; 而非SCI索引收录的中、英文 刊物发文量较少，分别仅发表了24篇和4篇。

由新西兰Magnolia出版社于2001年创立的专门 针对动物分类学的国际同行评审期刊Zootaxa发表 了130篇文献和 214 种及种下阶元新分类单元(占总 数的68\%)。该期刊发表周期短, 受到大多数分类学

表3 2020年发表10个以上新物种的学者统计

Table 3 Statistics of authors who published more than 10 new species for Hemiptera and 28 other orders of Insecta in 2020

\begin{tabular}{|c|c|c|c|c|}
\hline $\begin{array}{l}\text { 姓名 } \\
\text { Names }\end{array}$ & $\begin{array}{l}\text { 单位 } \\
\text { Institutions }\end{array}$ & $\begin{array}{l}\text { 关注类群 } \\
\text { Research groups }\end{array}$ & $\begin{array}{l}\text { 发文量 } \\
\text { No. of } \\
\text { publications }\end{array}$ & $\begin{array}{l}\text { 新种及亚种 } \\
\text { No. of species and } \\
\text { subspecies }\end{array}$ \\
\hline 张雅林 Yalin Zhang & $\begin{array}{l}\text { 西北农林科技大学 } \\
\text { Northwest A \& F University }\end{array}$ & $\begin{array}{l}\text { 半翅目、螳螂目 } \\
\text { Hemiptera and Mantodea }\end{array}$ & 10 & 33 \\
\hline 李卫海 Weihai Li & $\begin{array}{l}\text { 河南科技学院 } \\
\text { Henan Institute of Science and Technology }\end{array}$ & 襀翅目 Plecoptera & 22 & 28 \\
\hline 莫娆娆 Raorao Mo & 广西大学 Guangxi University & 襀翅目 Plecoptera & 14 & 17 \\
\hline 王国全 Guoquan Wang & 广西大学 Guangxi University & 襀翅目 Plecoptera & 13 & 16 \\
\hline 石福明 Fuming Shi & 河北大学 Hebei University & 直翅目 Orthoptera & 6 & 15 \\
\hline 陈祥盛 Xiangsheng Chen & 贵州大学 Guizhou University & 半翅目 Hemiptera & 8 & 14 \\
\hline 杨琳 Lin Yang & 贵州大学 Guizhou University & 半翅目 Hemiptera & 8 & 14 \\
\hline 杨定 Ding Yang & 中国农业大学 China Agricultural University & 襀翅目 Plecoptera & 10 & 13 \\
\hline 吴超 Chao Wu & $\begin{array}{l}\text { 中国科学院动物研究所 } \\
\text { Institute of Zoology, Chinese Academy of Sciences } \\
\text { 西南大学 Southwest University }\end{array}$ & $\begin{array}{l}\text { 直翅目、螳螂目 } \\
\text { Orthoptera and Mantodea } \\
\text { 蜚蠊目 Blattodea }\end{array}$ & 5 & 11 \\
\hline 陈志腾 Zhiteng Chen & $\begin{array}{l}\text { 江苏科技大学 } \\
\text { Jiangsu University of Science and Technology }\end{array}$ & 襀翅目 Plecoptera & 9 & 10 \\
\hline
\end{tabular}




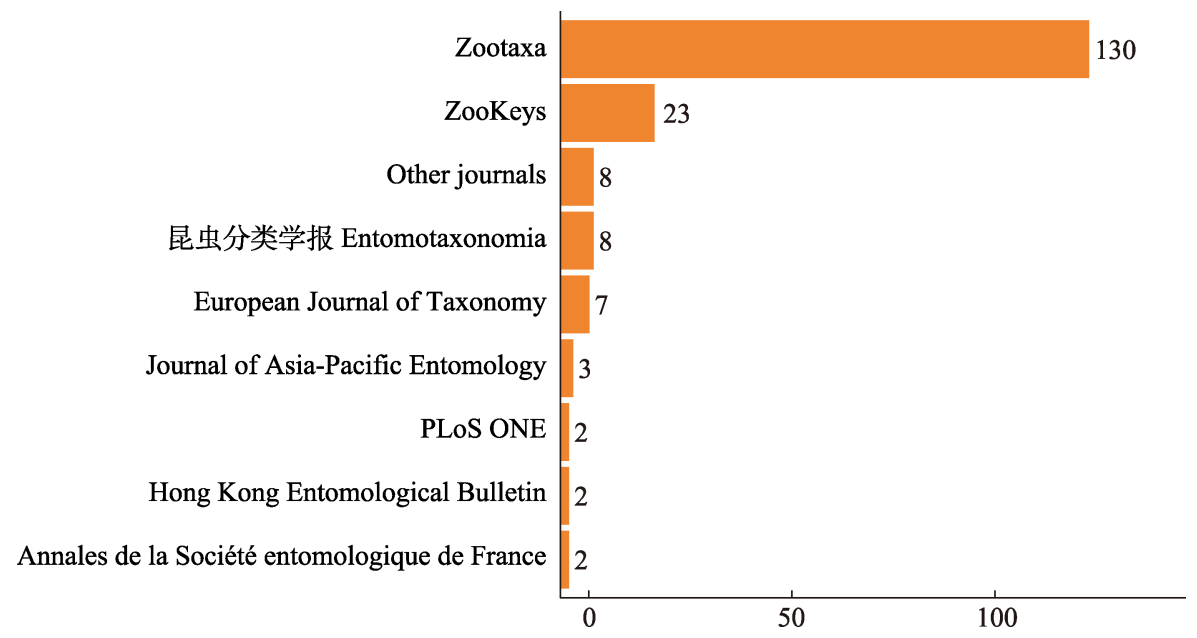

图32020年发表中国半翅目等29个目昆虫新分类单元的期刊

Fig. 3 The journals that published new taxa of Chinese Hemiptera and 28 other orders of Insecta in 2020

家的青睐, 为加速对生物多样性的认知和保护等做 出了非常重要的贡献。另一经典的分类学杂志 ZooKeys同样在发表新分类单元中扮演着重要的角 色，该刊物在2020年度共发表23篇文献，包括32个 新种。在非SCI索引的中文刊物中, 《昆虫分类学报》 在2020年度发文量最多。Hong Kong Entomological Bulletin发文量较少, 仅2篇, 但发表的新种最多, 这 两篇文献主要是对中国地区䗛目的长角枝亚科 (笛 䗛科)和蜼科的副厚䗛属(Parapachymorpha)进行了 非常系统的厘定(Ho et al, 2020a, b)。

综上，本文基于2020年所发表的中国半翅目等 29目昆虫的文献及其标本, 对隐藏在其中的多样化 的生物信息数据进行了挖掘和整理, 为进一步深入 研究物种演化的时空格局及生物多样性的保护提 供了基础。目前, 昆虫多样性的研究仍相对落后, 需加强培育中国昆虫分类学的研究队伍, 增强标本 采集等生物多样性调查与研究, 并积极挖掘生物标 本中隐藏的科学数据, 关注生物多样性信息学的研 究和发展, 推进数字化共享平台的建设。

致谢: 感谢中国科学院动物研究所研究生许双、徐 颖以及张潇璐在整理数据时给予的帮助。

\section{ORCID}

刘童祎 (D) https://orcid.org/0000-0002-6831-0537

乔格侠 (D) https://orcid.org/0000-0002-7300-6812

\section{参考文献}

Andrew C, Diez J, James TY, Kauserud H (2019) Fungarium specimens: A largely untapped source in global change biology and beyond. Philosophical Transactions of the Royal Society B: Biological Sciences, 374, 20170392.

Anonymous (2021) History of citation indexing. Clarivate Analytics. https://clarivate.com/webofsciencegroup/essays/ history-of-citation-indexing/. (accessed on 2021-03-16)

Barnosky AD, Matzke N, Tomiya S, Wogan GOU, Swartz B, Quental TB, Marshall C, McGuire JL, Lindsey EL, Maguire KC, Mersey B, Ferrer EA (2011) Has the Earth's sixth mass extinction already arrived? Nature, 471, 51-57.

Fujita MK, Leaché AD, Burbrink FT, McGuire JA, Moritz C (2012) Coalescent-based species delimitation in an integrative taxonomy. Trends in Ecology \& Evolution, 27, 480-488.

He P, Chen J, Kong HZ, Cai L, Qiao GX (2021) Important supporting role of biological specimen in biodiversity conservation and research. Bulletin of the Chinese Academy of Sciences, 36, 425-435. (in Chinese with English abstract) [贺鹏, 陈军, 孔宏智, 蔡磊, 乔格侠 (2021) 生物样本: 生物多样性研究与保护的重要支撑. 中国科学院院刊, 36, 425-435.]

He P, Chen J, Qiao GX (2019) Current situation and future of biological collections of Chinese Academy of Sciences. Bulletin of the Chinese Academy of Sciences, 34, 1359-1370. (in Chinese with English abstract) [贺鹏, 陈军, 乔格侠 (2019) 中国科学院生物标本馆(博物馆)的现状与 未来. 中国科学院院刊, 34, 1359-1370.]

Ho GWC (2020a) Contribution to the knowledge of Chinese Phasmatodea VI: New taxa and new nomenclature of the subfamily Necrosciinae from the Phasmatodea of China. Hong Kong Entomological Bulletin, 12(2), 3-28.

Ho GWC (2020b) Two new species of the genus 
Parapachymorpha Brunner von Wattenwyl, 1893 (Phasmida: Phasmatidae: Clitumninae: Medaurini) from China. Hong Kong Entomological Bulletin, 12(1), 3-9.

Lei FM, Song G, Cai TL, Qu YH, Jia CX, Zhao YF, Zhang DZ (2021) Research progress and prospect on biogeography of birds in China. Chinese Journal of Zoology, 56, 265-289. (in Chinese with English abstract) [雷富民, 宋刚, 蔡天龙, 屈延华, 贾陈喜, 赵义方, 张德志 (2021) 中国鸟类生物 地理学研究回顾与展望. 动物学杂志, 56, 265-289.]

Li XJ, Zhang DP, Yin HX (2020) Comparative analysis of mitogenomes among three species of Haplotropidini grasshoppers and a new species of the genus Sulcotropis (Orthoptera: Acridoidea: Pamphagidae) from China. Zootaxa, 4802, 541-555.

Mi X, Feng G, Hu Y, Zhang J, Chen L, Corlett RT, Hughes AC, Pimm S, Schmid B, Shi S, Svenning JC, Ma K (2021) The global significance of biodiversity science in China: An overview. National Science Review, 8, nwab032.

Molnar P, Boos WR, Battisti DS (2010) Orographic controls on climate and paleoclimate of Asia: Thermal and mechanical roles for the Tibetan Plateau. Annual Review of Earth and Planetary Sciences, 38, 77-102.

Nelson G, Ellis S (2019) The history and impact of digitization and digital data mobilization on biodiversity research. Philosophical Transactions of the Royal Society B: Biological Sciences, 374, 20170391.

Newbold T, Hudson LN, Hill SLL, Contu S, Lysenko I, Senior RA, Börger L, Bennett DJ, Choimes A, Collen B, Day J, De Palma A, Díaz S, Echeverria-Londoño S, Edgar MJ, Feldman A, Garon M, Harrison MLK, Alhusseini T, Ingram DJ, Itescu Y, Kattge J, Kemp V, Kirkpatrick L, Kleyer M, Correia DLP, Martin CD, Meiri S, Novosolov M, Pan Y, Phillips HRP, Purves DW, Robinson A, Simpson J, Tuck SL, Weiher E, White HJ, Ewers RM, Mace GM, Scharlemann JPW, Purvis A (2015) Global effects of land use on local terrestrial biodiversity. Nature, 520, 45-50.

Padial JM, Miralles A, De la Riva I, Vences M (2010) The integrative future of taxonomy. Frontiers in Zoology, 7, $1-14$.
Qian H, Jin Y, Ricklefs RE (2017) Phylogenetic diversity anomaly in angiosperms between eastern Asia and eastern North America. Proceedings of the National Academy of Sciences, USA, 114, 11452-11457.

Stork NE (2018) How many species of insects and other terrestrial arthropods are there on earth? Annual Review of Entomology, 63, 31-45.

Stork NE, McBroom J, Gely C, Hamilton AJ (2015) New approaches narrow global species estimates for beetles, insects, and terrestrial arthropods. Proceedings of the National Academy of Sciences, USA, 112, 7519-7523.

The Biodiversity Committee of Chinese Academy of Sciences (2020) Catalogue of Life China: 2020 Annual Checklist, Beijing, China. http://www.sp2000.org.cn/CoLChina. (accessed on 2021-03-16)

Wagner DL, Grames EM, Forister ML, Berenbaum MR, Stopak D (2021) Insect decline in the Anthropocene: Death by a thousand cuts. Proceedings of the National Academy of Sciences, USA, 118, e2023989118.

Wang X, Zhang FL, Zhang J (2017) Biodiversity information resources. I. Species distribution, catalogue, phylogeny, and life history traits. Biodiversity Science, 25, 1223-1238. (in Chinese with English abstract) [王昕, 张凤麟, 张健 (2017) 生物多样性信息资源. I. 物种分布、编目、系统发 育与生活史性状. 生物多样性, 25, 1223-1238.]

Wei FW, Nie YG, Miao HX, Lu H, Hu YB (2014) Advancements of the researches on biodiversity loss mechanisms. Chinese Science Bulletin, 59, 430-437. (in Chinese with English abstract) [魏辅文, 聂永刚, 苗海霞, 路浩, 胡义波 (2014) 生物多样性丧失机制研究进展. 科 学通报, 59, 430-437.]

Xing YW, Ree RH (2017) Uplift-driven diversification in the Hengduan Mountains, a temperate biodiversity hotspot. Proceedings of the National Academy of Sciences, USA, 114, E3444-E3451.

\section{附录 Supplementary Material}

附录1 中国半翅目等29目昆虫 2020 年度新种模式标本与文献名录

Appendix 1 List of type specimens and bibliography of new species of Chinese Hemiptera and 28 other orders of Insecta in 2020 https://www.biodiversity-science.net/fileup/PDF/2021200-1.pdf

\section{附录2 本研究所涉及模式标本保藏地点的中英文名称及缩写}

Appendix 2 List of abbreviation, Chinese and English names of deposit sites of type specimens in this study https://www.biodiversity-science.net/fileup/PDF/2021200-2.pdf 
刘童神, 陈静, 姜立云, 乔格侠 (2021) 中国半翅目等29目昆虫2020年新分类单元. 生物多样性, 29, 1050-1057. http://www.biodiversity-science.net/CN/10.17520/biods.2021200.

附录 1 中国半翅目等 29 目昆虫 2020 年度新种模式标本与文献名录

Appendix 1 List of type specimens and bibliography of new species of Chinese Hemiptera and 28 other orders of Insecta in 2020

\section{蜚蠊目 Blattodea}

Anaplecta arcuata Deng \& Che, 2020

Anaplectidae

Deng et al. 2020. European Journal of Taxonomy, 720: 77-106

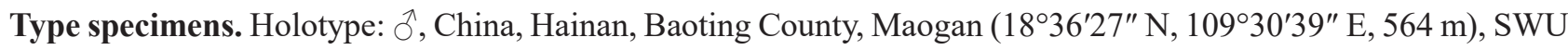
(标本存放地缩写: SWU, 见附录 2,下同). Paratypes: 1 ภ, 1 \% , same collection data as for holotype, SWU.

Anaplecta bicolor Deng \& Che, 2020

Deng et al. 2020. European Journal of Taxonomy, 720: 77-106

Type specimens. Holotype: ${ }^{\top}$, China, Yunnan, Xishuangbanna, Mengla County $\left(21^{\circ} 27^{\prime} 46^{\prime \prime}\right.$ N, $101^{\circ} 33^{\prime} 19^{\prime \prime}$ E, 668 m), SWU. Paratypes: 1ㅇ, China, Yunnan, same collection data as for holotype, SWU; $1 \delta^{\Uparrow} 1$ 으, Xishuangbanna,

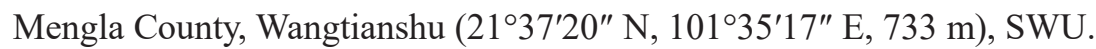

Anaplecta corneola Deng \& Che, 2020

Deng et al. 2020. European Journal of Taxonomy, 720: 77-106

Type specimens. Holotype: $\partial^{\top}$, Hainan, Ledong County, Mt. Jianfengling, Mingfeng Valley (18 $44^{\prime} 51^{\prime \prime} \mathrm{N}, 108^{\circ} 51^{\prime} 3^{\prime \prime}$ E, $798 \mathrm{~m}$ ), SWU. Paratypes: $1 \hat{\jmath}$, China, Hainan, same collection data as for holotype, SWU; $12 \hat{\jmath} 4$ 우우, same

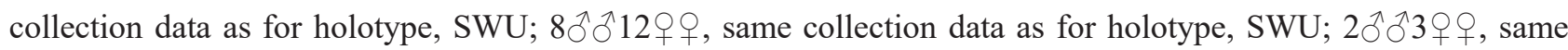
collection data as for holotype, SWU; $4 \delta^{\lambda} 3$ 우, Guangdong, Guangzhou City, Tianhe District, Longyandong

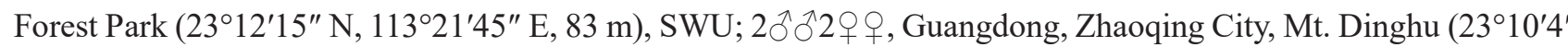

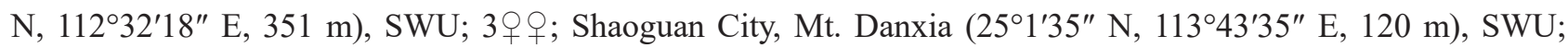

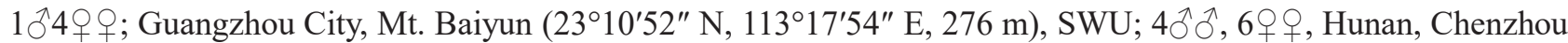

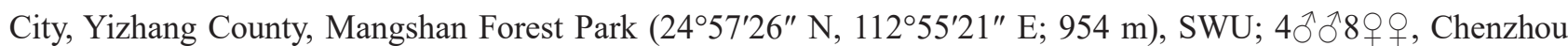

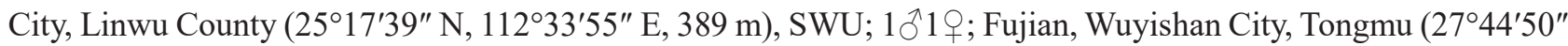
$\left.\mathrm{N}, 117^{\circ} 40^{\prime} 46^{\prime \prime} \mathrm{E}, 759 \mathrm{~m}\right), \mathrm{SWU}$.

Anaplecta cruciata Deng \& Che, 2020

Deng et al. 2020. European Journal of Taxonomy, 720: 77-106

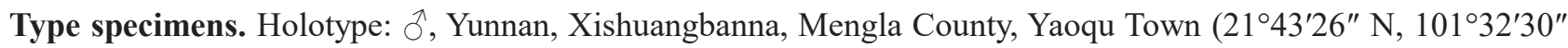
E; 820 m), SWU. Paratypes: 1 ${ }^{\prime}$; Yunnan, Xishuangbanna, Mengla County, Shangyong Town $\left(21^{\circ} 14^{\prime} 36^{\prime \prime}\right.$ N,

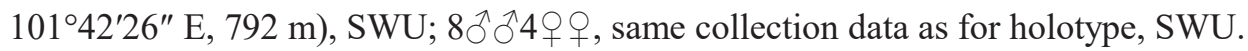

Anaplecta furcata Deng \& Che, 2020

Deng et al. 2020. European Journal of Taxonomy, 720: 77-106

Type specimens. Holotype: $\partial^{\lambda}$, Guangxi, Jinxiu County, Mt. Dayao $\left(24^{\circ} 08^{\prime} 26^{\prime \prime} \mathrm{N}, 110^{\circ} 11^{\prime} 42^{\prime \prime} \mathrm{E}, 944 \mathrm{~m}\right)$, SWU. Paratypes: $2 \hat{\jmath} \hat{\sigma}$, same collection data as for holotype, SWU.

Anaplecta nigra Deng \& Che, 2020

Deng et al. 2020. European Journal of Taxonomy, 720: 77-106

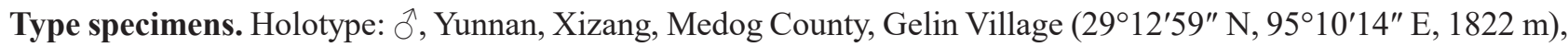
SWU. Paratype: 19 , same collection data as for holotype, SWU. 
刘童神, 陈静, 姜立云, 乔格侠 (2021) 中国半翅目等29目昆虫2020年新分类单元. 生物多样性, 29, 1050-1057. http://www.biodiversity-science.net/CN/10.17520/biods.2021200.

Anaplecta staminiformis Deng \& Che, 2020

Deng et al. 2020. European Journal of Taxonomy, 720: 77-106

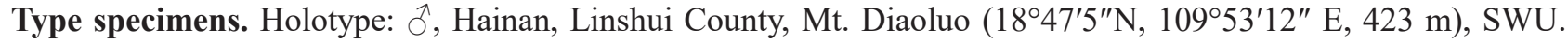
Paratypes: $1 \hat{\gamma}$, same collection data as for holotype, SWU. $2 \hat{\jmath} \delta^{\lambda}+ㅇ+$, Hainan, Ledong County, Mt. Jianfengling,

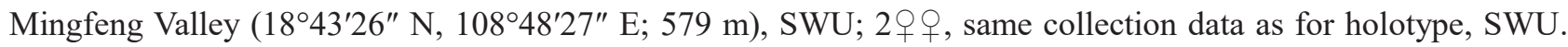

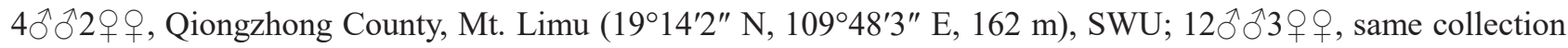
data, SWU; $1 \delta^{\Uparrow} 3$ 우, SWU.

Anaplecta strigata Deng \& Che, 2020

Deng et al. 2020. European Journal of Taxonomy, 720: 77-106

Type specimens. Holotype: $\hat{\partial}$, Hainan, Ledong County, Mt. Jianfengling, Mingfeng Valley $\left(18^{\circ} 43^{\prime 2} 26^{\prime \prime} \mathrm{N}\right.$,

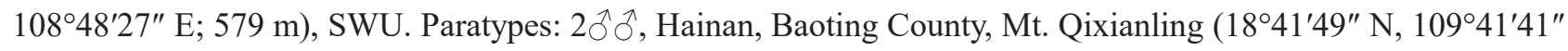
E, $231 \mathrm{~m}), \mathrm{SWU} ; 9^{\Uparrow} \partial^{\lambda} 2$ 우, same collection data as for holotype, SWU; $1 \overbrace{}^{\lambda}$, Hainan, Qiongzhong County $\left(19^{\circ} 2^{\prime 2} 22^{\prime \prime}\right.$

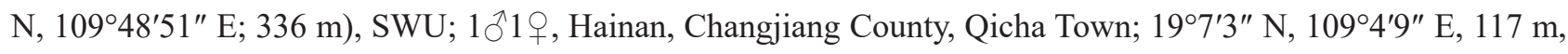

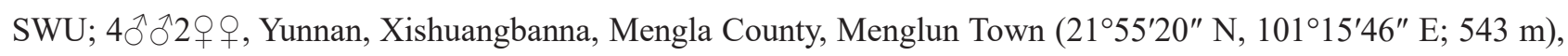
SWU.

Holocompsa zhangi Qiu, Wang \& Che, 2020

\section{Corydiidae}

Qiu et al. 2020. Annales de la Société Entomologique de France (N.S.)

Type specimens. Holotype: ${ }^{\lambda}$, China, Yunnan, Nongzhang Town, Yingiiang County, Dehong Prefecture $\left(24^{\circ} 36^{\prime} \mathrm{N}\right.$ $\left.97^{\circ} 53^{\prime} \mathrm{E}, 800 \mathrm{~m}\right)$, SWU.

\section{Ectobiidae}

Episymploce maxima Li, Liu, Qiu \& Yue, 2020

Li et al. 2020. ZooKeys, 954: 31-46 (2020)

Type specimens. Holotype: 1 $\hat{\jmath}$, China, Guangxi, Nonggang Village, Longzhou County, Chongzuo City $\left(22^{\circ} 28.26^{\prime} \mathrm{N}, 106^{\circ} 57.43^{\prime} \mathrm{E}\right)$. Allotype: 1 , , paratype: $1 \delta^{\Uparrow} 1$, , same data as holotype, ZCTC.

Episymploce sichuanensis Li, Liu, Qiu \& Yue, 2020

Li et al. 2020. ZooKeys, 954: 31-46 (2020)

Type specimens. Holotype: ${ }^{\lambda}$, China, Sichuan Daheishan, Panzhihua City $\left(26^{\circ} 38.48^{\prime} \mathrm{N}, 101^{\circ} 41.62^{\prime} \mathrm{E}\right)$. Allotype: 1 , , paratype: $7 \AA \widehat{s}$, same data as holotype, ZCTC.

Sigmella digitalis Li \& Wang, 2020

Li et al. 2020. PLoS One, 0232821

Type specimens. Holotype: $\delta^{\uparrow}$, China, Hainan, Mt. Bawangling ( $\left(9^{\circ} 05.1760\right.$ N, $109^{\circ} 07.3360$ E), SWU. Paratypes: $4 \overbrace{}^{\lambda}, 1$ female, same data as holotype; $4 \hat{\partial}$, China, Hainan, Liupancun, Jiyangzhen, Sanya $\left(18^{\circ} 14.8460 \mathrm{~N}\right.$ $\left.109^{\circ} 37.4820 \mathrm{E}\right)$, SWU.

Sigmella exserta Li \& Wang, 2020

Li et al. 2020. PLoS One, 0232821

Type specimens. Holotype: $\overbrace{}^{\lambda}$, China, Guangxi, Mt. Shengtangshan, Jinxiu, $\left(23^{\circ} 58.4140\right.$ N, $110^{\circ} 07.1680$ E, $\left.1182 \mathrm{~m}\right)$, SWU. Paratypes: $4 \hat{\jmath}$, same data as holotype. 
刘童神, 陈静, 姜立云, 乔格侠 (2021) 中国半翅目等29目昆虫2020年新分类单元. 生物多样性, 29, 1050-1057. http://www.biodiversity-science.net/CN/10.17520/biods.2021200.

Sigmella normalis Li \& Wang, 2020

Li et al. 2020. PLoS One, 0232821

Type specimens. Holotype: ${ }^{\top}$, China, Hainan, Mt. Wuzhishan ( $18^{\circ} 54.2900$ N, $109^{\circ} 41.0870$ E, 795 m), SWU. Paratypes: $4 \hat{\jmath} \widehat{\partial}$, same data as holotype; $5 \hat{\jmath} \widehat{\partial}$, China, Hainan, Mt. Diaoluoshan, Lingshui $\left(18^{\circ} 43.4300 \mathrm{~N}\right.$

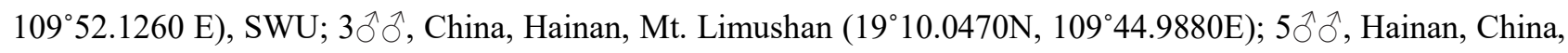
Mingfenggu, Mt. Jianfengling (1905.1760N, $109^{\circ} 07.3360$ E), SWU.

\section{弹尾目 Collembola}

Entomobryidae

Entomobrya tristriata Ma \& Chang, 2020

Ma et al. 2020. Zootaxa, 4731 (3): 335-346

Type specimens. Holotype: + , China, Jilin, Baishan City, Fusong County, Xianren Bridge Town (127'14'14'E, $\left.42^{\circ} 10^{\prime} 35^{\prime} \mathrm{N}\right)$, NU. Paratypes: 3 우, same data as holotype. 1 female on slide, the Gate of Changbai Mountain

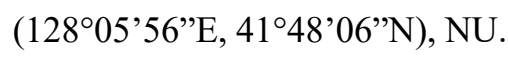

Sinodicranocentrus Zhang, 2020

Alloscopus bannaensis Zhang, 2020

Zhang et al. 2020. Arthropod Systematics and Phylogeny, 78 (1): $1-16$

Type specimens. Holotype: + , China, Yunnan, Mengla county, Menglun town, Xishuangbanna Tropical Botanical Garden $\left(21.919^{\circ} \mathrm{N}, 101.2709^{\circ} \mathrm{E}, 754 \mathrm{~m}\right)$, NJAU. Paratypes: 2 우, same data as holotype, NJAU.

Alloscopus liuae Zhang, 2020

Zhang et al. 2020. Arthropod Systematics and Phylogeny, 78 (1): $1-16$

Type specimens. Holotype: + , China, Guangdong, Guangzhou, Heshan Hilly Land Interdisciplinary Experimental Station, NJAU. Paratypes: $12 q+1 \widehat{\jmath}$, same data as holotype, NJAU.

\section{Isotomidae 等节䖴科}

Dimorphacanthella sichuanensis Gao \& Bu, 2020

Gao et al. 2020. Entomotaxonomia, 42(1): 1-5

Type specimens. Holotype: + , China, Sichuan, Litang County $\left(29^{\circ} 46^{\prime} \mathrm{N} 100^{\circ} 27^{\prime} \mathrm{E}, 3619 \mathrm{~m}\right)$, SNHM. Paratypes: $1{ }^{\lambda} 3$ 우, same data as holotype, SNHM.

Folsomia mongolica Huang \& Potapov, 2020

Potapov et al. 2020. ZooKeys, 935: 1-24

Type specimens. Holotype: + . China, Inner Mongolia Autonomous Region, Hulun Buir, New Barag Zuoqi, Xinbaoligexi Sumu, Bayin Chagan Nuori Lake, at shore of the saline lake $\left(48.38^{\circ} \mathrm{N}, 118.71^{\circ} \mathrm{E}, 669 \mathrm{~m}\right)$, SEMCAS. Paratypes: 20 specimens, same data as holotype: 10 specimens, SEMCAS, 5 specimens, MSPU, 5 specimens, SMNG.

Semicerura bryophila Potapov \& Sun, 2020

Potapov et al. 2020. Zootaxa, 4751 (1): 105-118

Type specimens. Holotype: $ᄋ$, China, Jilin, Changbai Mts $\left(41.758^{\circ} \mathrm{N}, 127.939^{\circ} \mathrm{E}\right.$, ca $\left.1750 \mathrm{~m}\right)$, IGACAS. Paratypes: 7 우으, China, Jilin, Changbai Mts $\left(41.847^{\circ} \mathrm{N}, 127.798^{\circ} \mathrm{E}\right.$, ca $\left.1100 \mathrm{~m}\right)$, IGACAS. 
刘童神, 陈静, 姜立云, 乔格侠 (2021) 中国半翅目等29目昆虫2020年新分类单元. 生物多样性, 29, 1050-1057. http://www.biodiversity-science.net/CN/10.17520/biods.2021200.

Semicerura draconis Potapov \& Sun, 2020

Potapov et al. 2020. Zootaxa, 4751 (1): 105-118

Type specimens. Holotype: + , China. Paratypes: 10 우, Jilin, Fusong County, Manjiang town forest (foothills of Changbai Mts), IGACA.

Onychiuridae

Leeonychiurus zijinensis Zhang, Sun \& Wu, 2020

Zhang et al. 2020. Zootaxa, 4743 (1): 137-143

Type specimens. Holotype: $\partial^{\lambda}$, China, Jiangsu, Nanjing, Zijinshan, Xiamafang Park, IGACAS. Paratypes: $4+q$ 1 $\partial^{\lambda}$, same data as holotype, IGACAS.

Tullbergiidae

Paratullbergia chuana Gao \& Bu, 2020

Gao \& Bu. 2020. Zootaxa, 4808 (1): 121-130

Type specimens. Holotype: $\widehat{\partial}$, China, Sichuan, Ganzi Tibetan Autonomous Region, Daocheng County, Xingmu

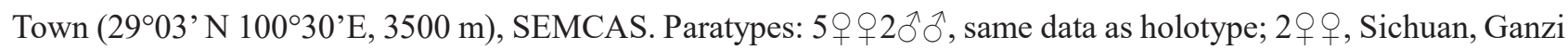
Region, Kangding City, Yala Town (3006’ N 10157’E, elev. 3100 m); 1옹, Sichuan, Ganzi Region, Litang County

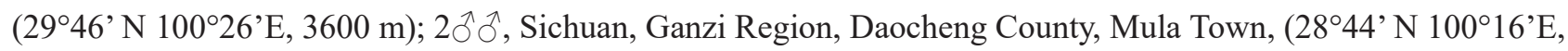

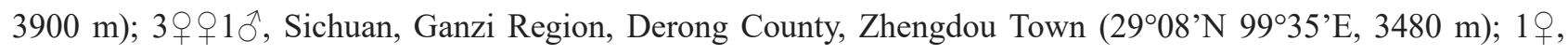
Sichuan, Ganzi Region, Daofu County $\left(30^{\circ} 40^{\prime} \mathrm{N} 101^{\circ} 17^{\prime} \mathrm{E}, 3550 \mathrm{~m}\right) ; 2+q 1 \widehat{O}^{\wedge}$, Sichuan, Ganzi Region, Danba County (30³6' N 101²0'E, 2940 m), SNHM, SEMCAS.

Neanuridae

Oudemansia chenorum Palacios-Vargas \& Bu, 2020

Palacios-Vargas \& Bu. 2020. Zootaxa, 4810 (1): 117-130

Type specimens. Holotype: + , China, Hainan, Sanya city, sandy beach of Ximaozhou island $\left(18^{\circ} 14^{\prime} \mathrm{N}, 109^{\circ} 22^{\prime} \mathrm{E}\right)$, SHNM. Paratypes: $1 \uparrow$, same data of holotype; $5 \circ \uparrow$, UNAM, SHNM; $1 \delta^{\lambda}$, China, Hainan, Sanya City, sandy beach of Ximaozhou Island $\left(18^{\circ} 14^{\prime} \mathrm{N}, 109^{\circ} 21^{\prime} \mathrm{E}\right)$, SHNM.

\section{革翅目 Dermaptera}

Chelisochidae

Chelisoches chongqingensis Ye \& Chen, 2020

Ye et al. 2020. Zootaxa, 4790 (3): 551-563

Type specimens. Holotype: $\widehat{\partial}$, China, Chongqin City, Simian Mountain, Feilong Temple, in surrounding lush vegetation (28.62 N, 106.39 E, 1000 m), ICJUST. Paratypes: $1{ }^{\top} 2$ 웅, same locality and data as holotype, ICJUST; 1 , China, Chongqin City, Jinfo Mountain (29.12 N, 107.17 E, 1600 m), ICJUST.

\section{蜉蝣目 Ephemeroptera}

Ephemerellidae

Cincticostella tornata Auychinda \& Gattolliat, 2020

Auychinda et al. 2020. Alpine Entomology, 4: 129-138 
刘童神, 陈静, 姜立云, 乔格侠 (2021) 中国半翅目等29目昆虫2020年新分类单元. 生物多样性, 29, 1050-1057. http://www.biodiversity-science.net/CN/10.17520/biods.2021200.

Type specimens. Holotype: Larva, China, Shaanxi, Hanzhong city, Foping county, Qinling Mts, slow forest brook in Panda valley $\left(33^{\circ} 40.368^{\prime} \mathrm{N}, 107^{\circ} 58.327^{\prime} \mathrm{E}, 1330 \mathrm{~m}\right)$, MZL. Paratypes: larvae, same data as for holotype, MZL.

\section{Heptageniidae}

Notacanthurus maculatus Zhou, 2020

Zhang et al. 2020. Zootaxa, 4802 (2): 335-348

Type specimens. Holotype: ${ }^{\top}$, China, Shaanxi, Fo-Ping county, Han-Zhong City, Yue-Ba town $\left(33^{\circ} 32^{\prime} 50.86^{\prime \prime} \mathrm{N}\right.$, $107^{\circ} 49^{\prime} 29.36^{\prime \prime E}$ ), NNU. Paratypes: $20^{\lambda}{ }^{\lambda}$, same as the holotype; China, Gansu, Tie-Lou village, Wen County, SaiKe bridge ( $\left.32^{\circ} 52^{\prime} 45.66^{\prime \prime} \mathrm{N}, 104^{\circ} 24^{\prime} 22.36^{\prime \prime} \mathrm{E}\right)$, NNU; 4 ${ }^{\circ}{ }^{\circ}$, China, Shanxi, Jin-Cheng City, Xia-Chuan Town, Xi-Xia

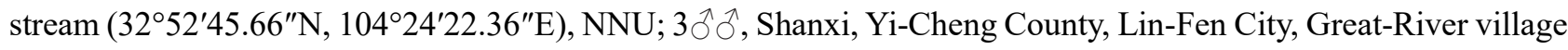
$\left(35^{\circ} 44^{\prime} 23.90^{\prime \prime} \mathrm{N}, 11^{\circ} 02^{\prime} 23.43^{\prime \prime} \mathrm{E}\right), \mathrm{NNU} ;{ }^{\top} \mathrm{o}^{\top}$, Hubei, Yi-Chang county, Yang-Lin-Qiao Town $\left(30^{\circ} 44^{\prime} 52.44^{\prime \prime} \mathrm{N}\right.$, $\left.110^{\circ} 45^{\prime} 57.85^{\prime \prime} \mathrm{E}\right), \mathrm{NNU}$.

Notacanthurus lamellosus Zhou, 2020

Zhang et al. 2020. Zootaxa, 4802 (2): 335-348

Type specimens. Holotype: ${ }^{\top}$, China, Hunan, Zhang-Jia-Jie City, Jin-Bian creek $\left(29^{\circ} 20^{\prime} 45.14^{\prime \prime} \mathrm{N}, 110^{\circ} 32^{\prime} 46.36^{\prime \prime} \mathrm{E}\right)$, NNU. Paratypes: $2 \hat{\partial} \partial^{2}$, same as the holotype; $1 \delta^{\lambda}$, Guizhou, Zun-Yi City, Jin-Sha village $\left(28^{\circ} 06^{\prime} 30.68^{\prime \prime} \mathrm{N}\right.$, $\left.107^{\circ} 41^{\prime} 35.78^{\prime \prime} \mathrm{E}\right), \mathrm{NNU}$.

Vietnamellidae

Vietnamella chebalingensis Tong, 2020

Luo et al. 2020. Zootaxa, 4868 (2): 208-220

Type specimens. Holotype: + , China, Gaungdong, Shixing County, Chebaling National Nature Reserve $\left(24^{\circ} 42^{\prime} 25^{\prime \prime} \mathrm{N}, 114^{\circ} 14^{\prime} 15^{\prime \prime} \mathrm{E}\right)$, SCAU. Paratypes: 6 larvae, same data as holotype, 3 larvae the same locality, one larva, SCAU.

\section{半翅目 Hemiptera}

Aphididae

Mesocallis platycaryae Qiao, 2020

Chen et al. 2020. ZooKeys, 1003: 19-30

Type specimens. Holotype: apterous viviparous female, China, Anhui, Yuexi County, Yaoluoping Reserve, Xiaoqiling (1100 m), IZCAS. Paratypes: 1 apterous viviparous female, 1 third instar nymph, and 1 fourth instar nymph, the collection data is the same as in the holotype, IZCAS.

Aleyrodidae

Aleuroclava schimae Wang, 2020

Wang et al. 2020. ZooKeys, 991: 121-128

Type specimens. Holotype: puparium, China, Zhejiang, Chun'an, Thousand Island Lake (29³1.21'N, $\left.118^{\circ} 52.41^{\prime} \mathrm{E}\right)$, ZAFU. Paratypes: 30 puparia, same data as holotype, ZAFU; 15 puparia, Zhejiang, Kaihua, Gutianshan Nature Reserve $\left(29^{\circ} 15.12^{\prime} \mathrm{N}, 118^{\circ} 06.42^{\prime} \mathrm{E}\right), \mathrm{ZAFU} ; 11$ puparia, Zhejiang, Jiangshan, Shuangxikou village, $\left(28^{\circ} 23.12^{\prime} \mathrm{N}\right.$, $\left.118^{\circ} 41.15^{\prime} \mathrm{E}\right)$, SEMCAS.

Aradidae

Neolibiocoris Bai, Heiss \& Cai, 2020 
刘童神, 陈静, 姜立云, 乔格侠 (2021) 中国半翅目等29目昆虫2020年新分类单元. 生物多样性, 29, 1050-1057. http://www.biodiversity-science.net/CN/10.17520/biods.2021200.

Neolibiocoris obtusus Bai, Heiss \& Cai, 2020

Surnaa et al. 2020. Zootaxa, 4885 (2): 235-248

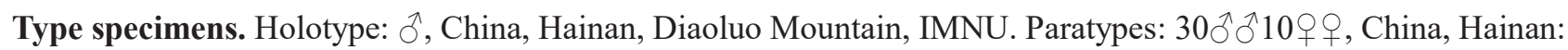

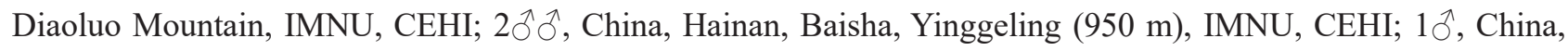
Hainan, Tongzha, Wuzhishan, IMNU; $2 \hat{\jmath} \widehat{\jmath}$, China, Hainan, Ledong, Jianfenling, IMNU, CEHI.

Rotundocoris pyramidalis Bai \& Heiss, 2020

Surnaa et al. 2020. Zootaxa, 4885 (2): 235-248

Type specimens. Holotype: $\widehat{\partial}$, China, Hainan, Baisha, Yinggeling (950 m), IMNU. Paratypes: 1q, China, Hainan, Baisha, Yinggeling (950 m), IMNU); 1ð̋, China, Hainan, Tongzha, Wuzhishan, IMNU.

Oblongocoris Bai, Heiss \& Cai, 2020

Oblongocoris hainanensis Bai, Heiss \& Cai, 2020

Shi et al. 2020. Zootaxa, 4820 (2): 385-390

Type specimens. Holotype: $\uparrow$, China, Hainan, Jianfeng, Tianchi, (IMNU. Paratype: $1 \uparrow$, same data as holotype, CEHI.

Paracremastaptera Bai, Heiss \& Cai, 2020

Paracremastaptera ximaensis Bai, Heiss \& Cai, 2020

Surnaa et al. 2020. Zootaxa, 4894 (3): 445-450

Type specimen. Holotype: ${ }^{\lambda}$, China, Yunnan, Yingiiang County, Xima Town, IMNU.

Paramorphocoris hubeiensis Bai \& Heiss, 2020

Wei et al. 2020. Entomotaxonomia, 42(4): 247-251

Type specimen. Holotype: + , China, Hubei, Wufeng, Yichang, IMNU.

Caliscelidae

Neosymplana Chen \& Gong, 2020

Neosymplana vittatum Gong, Yang \& Chen, 2020

Gong et al. 2020. Zootaxa, 4895 (3): 411-420

Type specimens. Holotype: đ, China, Yunnan, Yingjiang County, Yingjiang National Wetland Park $\left(24^{\circ} 69^{\prime} \mathrm{N}\right.$,

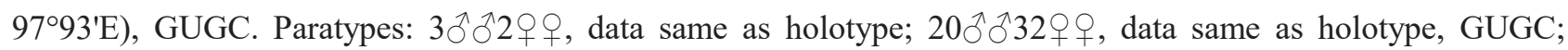
$2 \delta^{\lambda} 4$ 우 , China, Yunnan, Longchuan County, Chengguan ( $\left.24^{\circ} 33^{\prime} \mathrm{N}, 97^{\circ} 96^{\prime} \mathrm{E}\right), \mathrm{GUGC} ; 5 \delta^{\lambda} 8$ 우, China, Yunnan, Lianghe County, Mengyang Town $\left(24^{\circ} 78^{\prime} \mathrm{N}, 98^{\circ} 3^{\prime} \mathrm{E}\right)$, GUGC; $12 \partial^{\jmath} \partial^{2} 23^{\circ} \uparrow$, China, Yunnan, Ruili County, Wanding Town $\left(24^{\circ} \mathrm{N}, 97^{\circ} 83^{\prime} \mathrm{E}\right)$, GUGC.

Symplanella fulva Gong \& Chen, 2020

Gong et al. 2020. Zootaxa, 4801 (2): 355-362

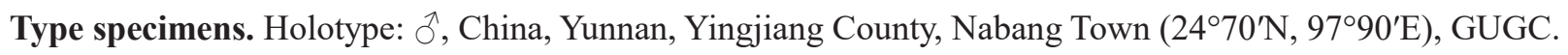

Symplanella nigricans Gong \& Chen, 2020

Gong et al. 2020. Zootaxa, 4801 (2): 355-362

Type specimens. Holotype: ${ }^{\top}$, China, Yunnan, Yingjiang County, Nabang Town $\left(24^{\circ} 70^{\prime} \mathrm{N}, 97^{\circ} 90^{\prime} \mathrm{E}\right)$, GUGC.

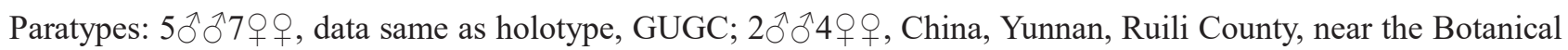


刘童神, 陈静, 姜立云, 乔格侠 (2021) 中国半翅目等29目昆虫2020年新分类单元. 生物多样性, 29, $1050-1057$. http://www.biodiversity-science.net/CN/10.17520/biods.2021200.

Garden $\left(24^{\circ} 01^{\prime} \mathrm{N}, 97^{\circ} 85^{\prime} \mathrm{E}\right)$, GUGC.

\section{Cicadellidae}

Balala fujiana Tang \& Zhang, 2020

Tang \& Zhang. 2020. Zootaxa, 4731 (1): 023-042

Type specimens. Holotype: $\widehat{\partial}$, China, Fujian, Jiuxian Mountain, NWAFU. Paratypes: $1 \hat{\jmath}$, same data as holotype; $2 \hat{\widehat{\partial}} \widehat{\jmath}$, China, Guangdong, Dadong Mountain, Lian County, SYSU.

Balala hainana Tang \& Zhang, 2020

Tang \& Zhang. 2020. Zootaxa, 4731 (1): 023-042

Type specimens. Holotype: $\hat{\jmath}$, China, Hainan, Jianfengling Mountain, CAF.

Daochia fenestrata Fang, Webb \& Xing, 2020

Fang et al. 2020. Zootaxa, 4758 (2): 378-386

Type specimens. Holotype: $1 \delta^{\lambda}$, China, Guizhou, Guiyang City, Forest park, GUGC; Paratypes: 2 + , Guizhou, Wangmo County, Dayi, GUGC; $3 \hat{\jmath} 1$, , Guizhou, Guiyang City, GUGC and BMNH; $7 \hat{\jmath} 4$ 우 holotype, GUGC; $1 \hat{\jmath}$, Guizhou, Libo County, Limingguan, GUGC; $2 \hat{\jmath} \widehat{\partial}$, Guizhou, Libo County, Maolan, GUGC; $1 \overbrace{}^{\lambda}$, Guizhou, Ziyun County, Zongdi, GUGC.

Dolichocaudus Wang \& Zhang, 2020

Dolichocaudus acicephalus Wang \& Zhang, 2020

Wang \& Zhang. 2020. Zootaxa, 4732 (1): 161-168

Type specimens. Holotype: $\widehat{\jmath}$, China, Guangxi, Banba village, Chengfang County (550 m), IZCAS. Paratype: 1 , China, Hainan, Ming-feng-gu, Jian-feng-ling (18 $44.658^{\prime}$ N108 $50.435^{\prime}$ E, $\left.1017 \mathrm{~m}\right)$, NWAFU.

Etmaria Cao \& Dmitriev, 2020

Etmaria magna Cao \& Dmitriev, 2020

Cao et al. 2020. Zootaxa, 4868 (3): 331-367

Type specimens. Holotype: ^̊, China, Taiwan, Taichung, Dashuishan Forest Rd. km 20, (24¹5'6"N, 12054'58"E, $1250 \mathrm{~m})$, TNMNS.

Etmaria triquetra Cao \& Zhang, 2020

Cao et al. 2020. Zootaxa, 4868 (3): 331-367

Type specimen. Holotype: ${ }^{\lambda}$, China, Yunnan, Yingjiang, Mangxian $\left(24^{\circ} 28^{\prime} 33^{\prime \prime} \mathrm{N}, 97^{\circ} 45^{\prime} 02^{\prime \prime} \mathrm{E}, 1089 \mathrm{~m}\right), \mathrm{NWAFU}$.

Etmaria ulterior Cao \& Zhang, 2020

Cao et al. 2020. Zootaxa, 4868 (3): 331-367

Type specimen. Holotype: ${ }^{\top}$, China, Yunnan, Yingjiang, Nabang (244ㄴ $\left.12^{\prime \prime} \mathrm{N}, 97^{\circ} 54^{\prime} 35^{\prime \prime} \mathrm{E}, 284 \mathrm{~m}\right)$, NWAFU.

Pseudothaia forcipis Cao \& Zhang, 2020

Cao et al. 2020. Zootaxa, 4868 (3): 331-367

Type specimens. Holotype: $\hat{\partial}$, China, Zhejiang, Mt. Wuyan, NWAFU. Paratypes: $1 \hat{\partial}$, same data as holotype; $1 \hat{\partial}$, China, Hunan, Mt. Heng, NWAFU. 
刘童神, 陈静, 姜立云, 乔格侠 (2021) 中国半翅目等29目昆虫2020年新分类单元. 生物多样性, 29, $1050-1057$. http://www.biodiversity-science.net/CN/10.17520/biods.2021200.

Mitjaevia dworakowskae Chen, Song \& Webb, 2020

Chen \& Song. 2020. ZooKeys, 964: 31-40

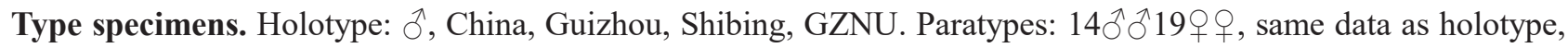
GZNU.

Mitjaevia shibingensis Chen, Song \& Webb, 2020

Chen \& Song. 2020. ZooKeys, 964: 31-40

Type specimens. Holotype: $\widehat{\jmath}$, China, Guizhou, Shibing, GZNU. Paratypes: $14 \overbrace{}^{\lambda} 55 \uparrow \uparrow$, same data as holotype, GZNU.

Empoascanara (Empoascanara) wengangensis Chen \& Song, 2020

Chen. \& Song. 2020. Zootaxa, 4780 (1): 191-196

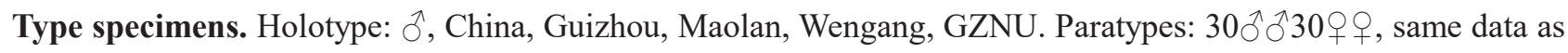
holotype, GZNU.

Thaia (Niema) xiaguensis Chen \& Song, 2020

Chen. \& Song. 2020. Zootaxa, 4780 (1): 191-196

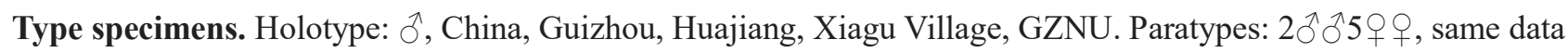
as holotype, GZNU.

Takagioma anguis Qin \& Huang, 2020

Qin et al. 2020. Zootaxa, 4742 (2): 299-310

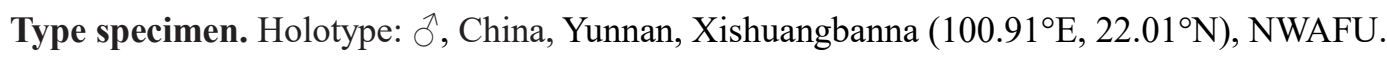

Takagioma curvata Kang \& Zhang, 2020

Qin et al. 2020. Zootaxa, 4742 (2): 299-310

Type specimens. Holotype: $\widehat{\jmath}$, China, Yunnan, Menglun $\left(101.28^{\circ} \mathrm{E}, 21.92^{\circ} \mathrm{N}\right)$, NWAFU. Paratype: $2 \hat{\jmath}$, same data as holotype; $4 \overbrace{}^{\Uparrow}$, Yunnan, Xishuangbanna $\left(100.91^{\circ} \mathrm{E}, 22.01^{\circ} \mathrm{N}\right), \mathrm{NWAFU}$.

Takagioma gladius Kang \& Zhang, 2020

Qin et al. 2020. Zootaxa, 4742 (2): 299-310

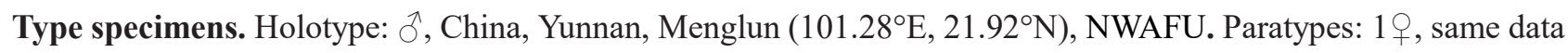
as holotype; $2 \delta^{\AA}$, Yunnan, Xishuangbanna $\left(100.91^{\circ} \mathrm{E}, 22.01^{\circ} \mathrm{N}\right), \mathrm{NWAFU}$.

Takagioma longicauda Qin \& Huang, 2020

Qin et al. 2020. Zootaxa, 4742 (2): 299-310

Type specimen. Holotype: $\overparen{\jmath}$, China, Yunnan, Xishuangbanna $\left(100.91^{\circ} \mathrm{E}, 22.01^{\circ} \mathrm{N}\right), \mathrm{NWAFU}$.

Takagioma pagoda Kang \& Zhang, 2020

Qin et al. 2020. Zootaxa, 4742 (2): 299-310

Type specimens. Holotype: $\sigma^{\Uparrow}$, China, Yunnan, Lincang, Sanchahe $\left(99.32^{\circ} \mathrm{E}, 24.08^{\circ} \mathrm{N}\right)$, NWAFU. Paratype: $1 \overbrace{}^{\Uparrow}$, same data as holotype, NWAFU.

Takagioma rostra Qin \& Huang, 2020 
刘童神, 陈静, 姜立云, 乔格侠 (2021) 中国半翅目等29目昆虫2020年新分类单元. 生物多样性, 29, 1050-1057. http://www.biodiversity-science.net/CN/10.17520/biods.2021200.

Qin et al. 2020. Zootaxa 4742 (2): 299-310

Type specimens. Holotype: $\partial^{\lambda}$, China, Yunnan, Xishuangbanna $\left(100.90^{\circ} \mathrm{E}, 22.03^{\circ} \mathrm{N}\right)$, NWAFU. Paratype: $2 \delta^{\lambda}$, collect data same as holotype, NWAFU.

Seriana bacilla Tan, Yuan \& Song, 2020

Tan et al. 2020. Zootaxa, 4728 (2): 275-282

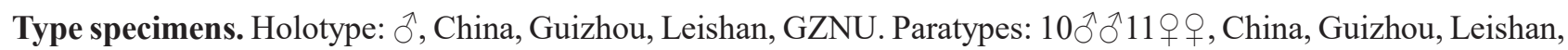

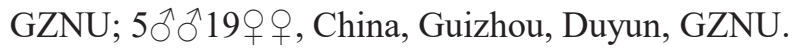

Singapora shiqianensis Li \& Zhang, 2020

Li \& Zhang. 2020. Zootaxa, 4808 (2): 365-372

Type specimens. Holotype: $\hat{\partial}$, China, Guizhou, Tongren, Shiqian, NWAFU. Paratypes: $5 \hat{\jmath}(1+$, same data as holotype; $2 \widehat{\jmath} 3$ 웅, China, Guizhou, Tongren, Shiqian, NWAFU.

Uniformus Jiao \& Yang, 2020

Uniformus inclusus Jiao \& Yang, 2020

Jiao \& Yang, 2020. Zootaxa, 4868 (4): 596-600

Type specimens. Holotype: $\curvearrowright$, China, Yunnan, Jinghong (608 m), GUGC. Paratype: $1 \curvearrowright$, Yunnan, Mohan (690 m), GUGC.

Uniformus rostellatus Jiao \& Yang, 2020

Jiao \& Yang, 2020. Zootaxa, 4868 (4): 596-600

Type specimens. Holotype: $\widehat{\partial}$, China, Yunnan, Lvchun, GUGC. Paratype, $1 \widehat{\jmath}$, China, Yunnan, Pingbian (2044 m), GUGC.

Thagria denticosta Wang, Li \& Dai, 2020

Wang et al. 2020. Zootaxa, 4821 (2): 363-370

Type specimens. Holotype: $\hat{\partial}$, China, Guizhou, Anlong County, GUGC. Paratype: $1 \hat{\jmath}$, China, Guangxi, Tian'e County, GUGC.

Thagria fimbriata Wang, Li \& Dai, 2020

Wang et al. 2020. Zootaxa, 4821 (2): 363-370

Type specimens. Holotype: $\widehat{\partial}$, China, Yunnan, Bao Shan County, GUGC. Paratype: $1 \partial^{\lambda}$, same data as holotype, GUGC.

Thagria trifurcata Wang, Li \& Dai, 2020

Wang et al. 2020. Zootaxa, 4821 (2): 363-370

Type specimens. Holotype: ${ }^{\AA}$, China, Guangxi, Damingshan, GUGC. Paratypes: 1 q , same data as holotype, GUGC.

Parascaphoidella Wei, Fang \& Xing, 2020

Parascaphoidella biprocessa Wei, Fang \& Xing, 2020

Wei et al. 2020. Zootaxa, 4810 (2): 328-334

Type specimen. Holotype: $\widehat{\jmath}$, China, Guangxi, Tian'e County, Longtan Natural Reserve, GUGC. 
刘童神, 陈静, 姜立云, 乔格侠 (2021) 中国半翅目等29目昆虫2020年新分类单元. 生物多样性, 29, 1050-1057. http://www.biodiversity-science.net/CN/10.17520/biods.2021200.

Parabolopona yunnanensis Xu \& Zhang, 2020

Xu \& Zhang. 2020. Zootaxa, 4821 (1): 189-195

Type specimens. Holotype: $\hat{\jmath}$, Yunnan, Mengla County, Shangyong Town, NWAFU. Paratype: $1 \uparrow$, data same as holotype, NWAFU.

Reticuluma bipennata Xu, Yu, Dai \& Yang, 2020

Xu et al. 2020. Zootaxa, 4809 (1): 177-186

Type specimens. Holotype: $\widehat{\partial}$, China, Yunnan , Mt. Gaoligong, Baihua Ridge, GUGC; Paratypes: $1 \hat{\jmath}$, China,

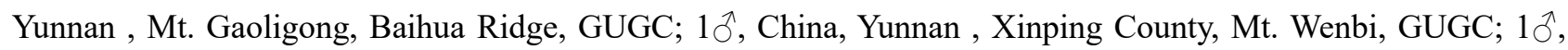
China, Yunnan, Mengla County, Menglun Town, GUGC.

Fanjinga Yu \& Yang, 2020

Fanjinga digitata Yu \& Yang, 2020

Yu et al. 2020. ZooKeys, 908: 39-44

Type specimens. Holotype: $\widehat{\partial}$, China, Guizhou, Fanjing Mountain, GUGC. Paratypes: $3 \hat{\partial} \widehat{\partial}$, same collecting data as holotype, GUGC, GZNU.

Watanabella curvatua Zhang \& Xing, 2020

Zhang \& Xing. 2020. Zootaxa, 4808, 539-546.

Type specimen. Holotype: $\widehat{\jmath}$, China, Yunnan, Lushui County, Pianma, GUGC.

Limassolla bicruralis Huang \& Zhang, 2020

Zhou et al. 2020. Zootaxa, 4878 (3): 559-571

Type specimens. Holotype: $\widehat{\partial}$, China, Yunnan, Sanchahe, NWAFU. Paratypes: $6 \AA \hat{\jmath} 4 ㅇ+q$, same data as holotype, NWAFU.

Limassolla kunyica Huang \& Zhang, 2020

Zhou et al. 2020. Zootaxa, 4878 (3): 559-571

Type specimens. Holotype: $\widehat{\partial}$, China, Shandong, Yantai, Mt. Kunyu (500 m), NWAFU. Paratypes: $14 \AA \widehat{\jmath} 18$ 우, same data as holotype, NWAFU.

Limassolla uncata Huang \& Zhang, 2020

Zhou et al. 2020. Zootaxa, 4878 (3): 559-571

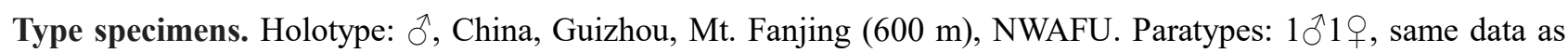
holotype, NWAFU.

Limassolla nigropunctata Huang \& Zhang, 2020

Zhou et al. 2020. Zootaxa, 4878 (3): 559-571

Type specimens. Holotype: $\widehat{\partial}$, China, Guangxi, Tongzhonglinchang, NWAFU. Paratypes: $1 \delta 2 \propto+\uparrow$, same data as holotype, NWAFU.

Limassolla spinulata Huang \& Zhang, 2020

Zhou et al. 2020. Zootaxa, 4878 (3): 559-571

Type specimens. Holotype: $\hat{\sigma}$, China, Yunnan, Sanchahe, NWAFU. Paratypes: $6 \hat{\jmath} \widehat{\delta} 1 \propto+$, same data as holotype; 
刘童神, 陈静, 姜立云, 乔格侠 (2021) 中国半翅目等29目昆虫2020年新分类单元. 生物多样性, 29, 1050-1057. http://www.biodiversity-science.net/CN/10.17520/biods.2021200.

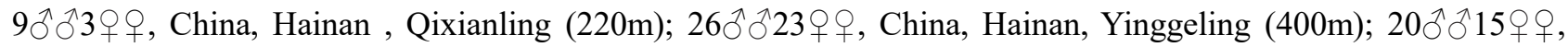
China, Hainan, Mt. Wuzhi, Maoyang village (325 m); 7ふぇ , China, Hainan, Jianfeng village (170m), NWAFU.

Paramritodus Xue \& Zhang, 2020

Paramritodus introflexus Xue \& Zhang, 2020

Xue \& Zhang. 2020. Zoological Journal of the Linnean Society, 189, 1438-1463

Type specimens. Holotype: $\hat{\partial}$, China, Yunnan, Baoshan, NWAFU. Paratypes: $3 \hat{\partial} \hat{\partial}$, same data as holotype, NWAFU.

Paramritodus spatiosus Xue \& Zhang, 2020

Xue \& Zhang. 2020. Zoological Journal of the Linnean Society, 189, 1438-1463

Type specimen. Holotype: $\widehat{\partial}$, China, Yunnan, Dali, Yangbi, Yangjiang, NWAFU.

Paramritodus triangulus Xue \& Zhang, 2020

Xue \& Zhang. 2020. Zoological Journal of the Linnean Society, 189, 1438-1463

Type specimens. Holotype: $\widehat{\partial}$, China, Yunnan, Nansan, NWAFU. Paratypes: $5 \widehat{\delta} \delta^{\lambda}+q$, same data as holotype, NWAFU.

\section{Cicadidae}

Versicolora Wei, Wang, Hayashi, He \& Pham, 2020

Versicolora bellula Wei, Wang, Hayashi, He \& Pham, 2020

Wei et al. 2020. Zootaxa, 4759 (2): 277-286

Type specimens. Holotype: $\widehat{\partial}$, China, Yunnan, Luchun County, NWAFU. Paratypes: $1 \hat{\partial}$, China, Yunnan, Luchun County, NWAFU; 1 , , China, Yunnan, Pingbian County, NWAFU; $1 \overbrace{}^{\Uparrow}$, NSMT.

Versicolora ziyongi Wei, Wang, Hayashi, He \& Pham, 2020

Wei et al. 2020. Zootaxa, 4759 (2): 277-286

Type specimens. Holotype: $\widehat{\alpha}$, China, Guangdong, Mt. Jiulian, Lianping County, Heyuan City, NWAFU. Paratypes:

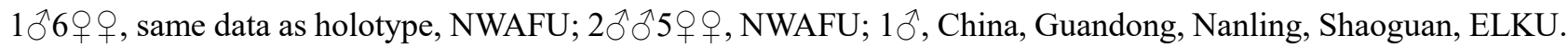

\section{Cixiidae}

Andixius cultratus Wang, Zhi \& Chen, 2020

Wang et al. 2020. Zootaxa, 4802 (3): 440-448

Type specimens. Holotype: $\sigma^{\AA}$, China, Guangdong, Shixing County, Chebaling National Natural Reserve $\left(24^{\circ} 43^{\prime} \mathrm{N}\right.$, $\left.114^{\circ} 15^{`} \mathrm{E}\right)$, GUGC; Paratypes: $23{ }^{\lambda} \partial^{\Uparrow} 16$ 웅, same data as holotype, GUGC.

Andixius lingulatus Wang, Zhi \& Chen, 2020

Wang et al. 2020. Zootaxa, 4802 (3): 440-448

Type specimens. Holotype: ${ }^{\lambda}$, China, Guangxi, Jinxiu County, Dayaoshan National Natural Reserve $\left(24^{\circ} 08^{\prime} \mathrm{N}\right.$, $\left.110^{\circ} 11^{\prime} \mathrm{E}\right)$, GUGC. Paratypes: $3{ }^{\lambda} \delta^{\lambda} 4$ 우 , same data as holotype, GUGC.

Betacixius latissimus Zhi \& Chen, 2020

Zhi et al. 2020. ZooKeys, 956: 1-18

Type specimens. Holotype: ${ }^{\top}$, China, Yunnan, Jinping County, Fenshuiling National Nature Reserve $\left(22^{\circ} 46^{\prime} \mathrm{N}\right.$, 
刘童神, 陈静, 姜立云, 乔格侠 (2021) 中国半翅目等29目昆虫2020年新分类单元. 生物多样性, 29, $1050-1057$. http://www.biodiversity-science.net/CN/10.17520/biods.2021200.

$\left.103^{\circ} 13^{\prime} \mathrm{E}\right)$, GUGC; Paratypes: $1{ }^{\wedge} 1$ \% , same data as holotype, GUGC.

Betacixius maguanensis Zhi \& Chen, 2020

Zhi et al. 2020. ZooKeys, 956: 1-18

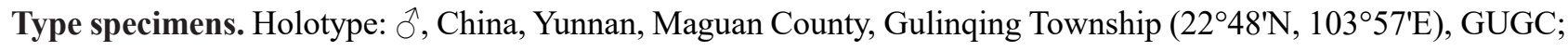
Paratypes: $8 \hat{\jmath} \widehat{\delta}$ 우우, same data as holotype, GUGC.

Indolipa fugongensis Zhi \& Chen, 2020

Zhi et al. 2020. ZooKeys, 956: 19-30

Type specimens. Holotype: $\partial^{\lambda}$, China, Yunnan, Fugong County $\left(26^{\circ} 54^{\prime} \mathrm{N}, 98^{\circ} 52^{\prime} \mathrm{E}\right)$, GUGC. Paratypes: $7 \delta^{\lambda} 6$ 우우, GUGC.

Indolipa longlingensis Zhi \& Chen, 2020

Zhi et al. 2020. ZooKeys, 956: 19-30

Type specimens. Holotype: $\widehat{O}^{\lambda}$, China, Yunnan, Longling County $\left(24^{\circ} 35^{\prime} \mathrm{N}, 98^{\circ} 41^{\prime} \mathrm{E}\right)$, GUGC. Paratypes: $22 \widehat{ð} 25$ 우우, same data as holotype, GUGC.

Oliparisca menglaensis Zhi \& Chen, 2020

Zhi et al. 2020. Zootaxa, 4896, (4): 577-585

Type specimens. Holotype: $\overbrace{}^{\top}$, China, Yunnan, Mengla County, Mohan Town $\left(21^{\circ} 14^{\prime}, 101^{\circ} 42^{\prime}\right)$, GUGC. Paratypes: $1 \delta^{\lambda} 6$ 우, same collection area as holotype, GUGC.

\section{Coccoidea}

Pulvinaria pistaciae Cao \& Feng, 2020

Cao T. \& Feng. 2020. Zootaxa, 4750 (2): 225-236

Type specimens. Holotype: $q$, China, Yunnan, Kunming, NWAFU. Paratypes: $9 q+$, same data as holotype, NWAFU.

Ortheziidae

Nipponorthezia guizhouensis Zheng \& Xing, 2020

Zheng \& Xing. Zootaxa, 4786 (3): 444-450

Type specimens. Holotype: $\uparrow$, China, Guizhou, Guiyang City, Wudang, Yangchang, GUGC. Paratypes: $2 q \uparrow$, China, Guizhou, Yanhe County, GUGC; $2 q+$, China, Guizhou, Dafang County, GUGC; $10 q+$, China, Guizhou, Fenggang County, GUGC.

Neobelocera biprocessa Li, Yang \& Chen, 2020

Delphacidae

Li et al. 2020. European Journal of Taxonomy, 641: 1-14.

Type specimens. Holotype: $\widehat{\jmath}$, China, Hainan, Changjiang County $\left(19^{\circ} 06^{\prime} \mathrm{N}, 109^{\circ} 04^{\prime} \mathrm{E}\right)$, GUGC. Paratypes: $1 \delta 2 ㅇ ㅜ$, same collection data as for holotype, GUGC.

Neobelocera russa Li, Yang \& Chen, 2020

Li et al. 2020. European Journal of Taxonomy, 641: 1-14.

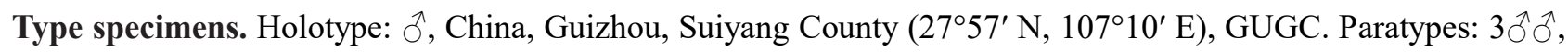


刘童神, 陈静, 姜立云, 乔格侠 (2021) 中国半翅目等29目昆虫2020年新分类单元. 生物多样性, 29, 1050-1057. http://www.biodiversity-science.net/CN/10.17520/biods.2021200.

same collection data as for holotype, GUGC.

\section{Diaspididae}

Fiorinia yongxingensis Liu, Cai \& Feng, 2020

Liu et al. 2020. Zootaxa, 4729 (3): 388-400

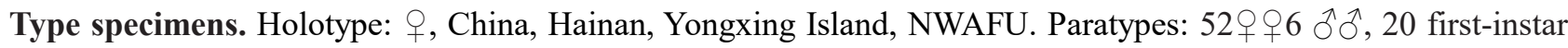
nymphs, 5 second-instar female nymphs, 15 second-instar male nymphs, 2 third-instar female nymphs, same data as holotype, NWAFU; 27 adult females, 16 adult males, 16 second-instar male nymphs, 4 first-instar nymphs, China, Hainan, Haikou City, Meilan District, NWAFU.

Flatidae

Atracis ocularia Ai, Peng \& Zhang, 2020

Ai et al. 2020. European Journal of Taxonomy, 717: 108-128

Type specimen. Holotype: $\partial^{\lambda}$, China, Hainan, Mt Jianfengling, NWAFU.

Atracis patefacta Ai, Peng \& Zhang, 2020

Ai et al. 2020. European Journal of Taxonomy, 717: 108-128

Type specimens. Holotype: $\hat{\jmath}$, China, Yunnan, Mengyang, Wild elephants Valley $(800 \mathrm{~m})$, NWAFU. Paratype: 1 , China, Yunnan, Banna, Menglong, NWAFU.

Atracis punctulata Ai, Peng \& Zhang, 2020

Ai et al. 2020. European Journal of Taxonomy, 717: 108-128

Type specimen. Holotype: ${ }^{\top}$, China, Guangdong, Mt Nanling, NWAFU.

Atracis sphaerica Ai, Peng \& Zhang, 2020

Ai et al. 2020. European Journal of Taxonomy, 717: 108-128

Type specimens. Holotype: $\overbrace{}^{\lambda}$, China, Guangxi, Longzhou, Nonggang, NWAFU. Paratypes: 1 , , China, Guangxi, Mt Daqingshan, BMNHC; 1 , China, Zhejiang, Mt Wuyanling, NWAFU.

Atracis ungulata Ai, Peng \& Zhang, 2020

Ai et al. 2020. European Journal of Taxonomy, 717: 108-128

Type specimens. Holotype: $\hat{\jmath}$, China, Hainan, Mt Jianfengling, CAF. Paratypes: $1 \hat{\jmath}$, China, Hainan, NWAFU; $1 \hat{\jmath}$, China, Hainan, Mt Jianfengling, Tianchi, SYSU.

\section{Fulgoridae}

Limois sordida Wang, Xu, Constant \& Qin, 2020

Wang et al. 2020. European Journal of Taxonomy, 720: 35-61

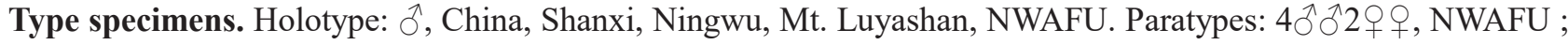
$2 q \circ$, Beijing, Mt. Baihuashan, Huang Tuo village, CAU; 1§, Beijing, Mt. Baihuashan, Huang Tuo village, CAU; 1§, Beijing, Mt. Baihuashan, CAU; 10, Hebei, Mt. Xiaowutaishan, IZCAS.

Issidae

Eusarimissus Wang \& Bourgoin, 2020

Eusarimissus hezhouensis Wang \& Bourgoin, 2020 
刘童神, 陈静, 姜立云, 乔格侠 (2021) 中国半翅目等29目昆虫2020年新分类单元. 生物多样性, 29, 1050-1057. http://www.biodiversity-science.net/CN/10.17520/biods.2021200.

Wang \& Bourgoin. 2020. ZooKeys, 912: 13-23

Type specimens. Holotype: ${ }^{\top}$, China, Guangxi, Hezhou, Qichong Nature Reserve $\left(24^{\circ} 13^{\prime} 6^{\prime \prime N}, 110^{\circ} 48^{\prime} 34^{\prime \prime E}, 180\right.$

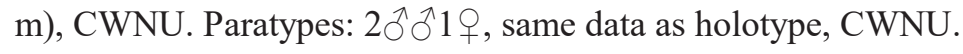

Kodaianella furcata Chang \& Chen, 2020

Chang et al. 2020. ZooKeys, 917: 85-104

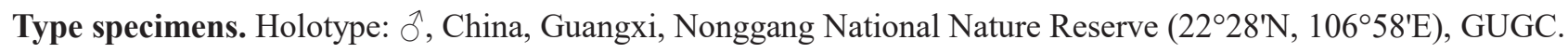
Paratypes: $5 \hat{\partial} \hat{\partial}$, same data as holotype, GUGC; $1 \hat{\partial}$, Guangxi, Nonggang National Nature Reserve $\left(22^{\circ} 28^{\prime} \mathrm{N}\right.$, $\left.106^{\circ} 58^{\prime} \mathrm{E}\right)$, GUGC.

Pusulissus Bourgoin \& Wang, 2020

Pusulissus coronomensis Bourgoin \& Wang, 2020

Bourgoin, \& Wang. 2020. ZooKeys, 997: 69-94

Type specimens. Holotype: ${ }_{0}$, China, Guangxi, Hezhou, Qichong Natural Reserve $\left(24^{\circ} 13^{\prime} 6^{\prime \prime} \mathrm{N}, 110^{\circ} 48^{\prime} 34^{\prime \prime E}, 180\right.$ m), CWNU. Paratypes: $1 \precsim 1$, same data as holotype, CWNU.

Sivaloka arcuata Chang \& Chen, 2020

Chang et al. 2020. ZooKeys, 917: 85-104

Type specimens. Holotype: $\widehat{\partial}$, China, Guizhou, Anlong County, Xianheping Provincial Nature Reserve $\left(22^{\circ} 59^{\prime} \mathrm{N}\right.$,

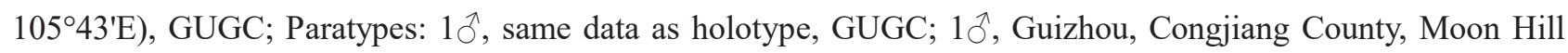
$\left(25^{\circ} 38^{\prime} \mathrm{N}, 108^{\circ} 13^{\prime} \mathrm{E}, 1159 \mathrm{~m}\right)$, GUGC.

Sivaloka trigona Chang \& Chen, 2020

Chang et al. 2020. ZooKeys, 917: 85-104

Type specimen. Holotype: ${ }^{\lambda}$, China, Guangxi, Yangshuo County (2459'N, 105³6'E), GUGC.

Tempsarima Chang \& Chen, 2020

Tempsarima bipunctata Chang \& Chen, 2020

Chang et al. 2020. ZooKeys, 956: 31-47

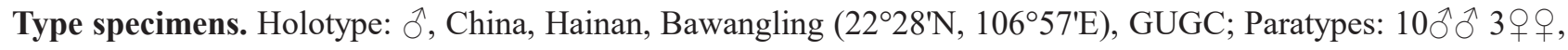

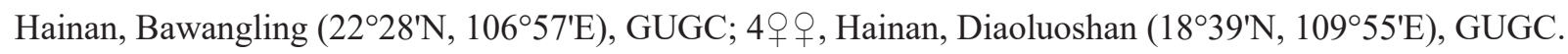

Tetrichina Chang \& Chen, 2020

Tetrichina trihamulata Chang \& Chen, 2020

Chang et al. 2020. ZooKeys, 956: 31-47

Type specimens. Holotype: $\overbrace{}^{\Uparrow}$, China, Hainan, Jianfengling National Park (18 $42^{\prime} \mathrm{N}, 108^{\circ} 51^{\prime} \mathrm{E})$, GUGC; Paratypes:

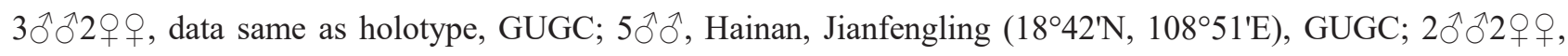

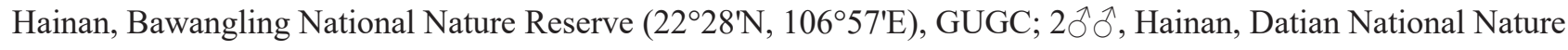
Reserve $\left(19^{\circ} 06^{\prime} \mathrm{N}, 108^{\circ} 47^{\prime} \mathrm{E}\right)$, GUGC.

Tetrichina fuscovinclum Wang \& Bourgoin Wang \& Bourgoin, 2020

Wang et al. 2020. Zootaxa, 4858 (4): 589-600

Type specimens. Holotype: $\widehat{\partial}$, China, Hainan, Yinggeling, Yinggezui (N 190 03.049', E 109 33.751', 693 m), IZCAS. Paratype: 1 , Hainan, Jianfengling, NWAFU. 
刘童神, 陈静, 姜立云, 乔格侠 (2021) 中国半翅目等29目昆虫2020年新分类单元. 生物多样性, 29, 1050-1057. http://www.biodiversity-science.net/CN/10.17520/biods.2021200.

Microsarimodes flavomaculata Wang \& Bourgoin, 2020

Wang et al. 2020. Zootaxa, 4858 (4): 589-600

Type specimen. Holotype: $\widehat{\partial}$, China, Yunnan, Mangshi, NWAFU.

Malcidae

Malcus alatus Wang \& Bu, 2020

Wang \& Bu. 2020. Zootaxa, 4759 (1): 031-048

Type specimen. Holotype: $\odot$, China, Yunnan, Tengchong County, NKU.

Malcus bacillus Wang \& $\mathrm{Bu}, 2020$

Wang \& Bu. 2020. Zootaxa, 4759 (1): 031-048

Type specimens. Holotype: $\widehat{\partial}$, China, Yunnan, Dehong Prefecture, Yingjiang County, Tongbiguan Township (1531m-1790m), NKU. Paratype: $1 \AA$, same locality as holotype, $1530 \mathrm{~m}$, NKU.

Malcus externus Wang \& Bu, 2020

Wang \& Bu. 2020. Zootaxa, 4759 (1): 031-048

Type specimens. Holotype: $\widehat{\jmath}$, China, Tibet, Linzhi City, Medog County, NKU. Paratypes: $2 q q$, same data as the holotype, NKU.

Malcus planus Wang \& Bu, 2020

Wang \& Bu. 2020. Zootaxa, 4759 (1): 031-048

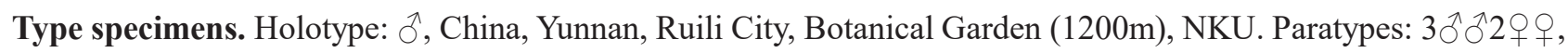
same data as the holotype, NKU.

Malcus zhengi Wang \& Bu, 2020

Wang \& Bu. 2020. Entomotaxonomia, 42(4): 252-257

Type specimens. Holotype: $\widehat{\partial}$, China, Tibet, Nyingchi City, Zayu County, next to the highway of Ping'an Hotel (97.017E 28.5014N, $1254 \mathrm{~m}$ ), NKU. Paratypes: $1 \delta^{\Uparrow} 5$ 우, same data as the holotype, NKU.

Meenoplidae

Metanigrus rotundatus Liu \& Qin, 2020

Liu \& Qin. 2020. Entomotaxonomia, 42 (1): 25-32

Type specimens. Holotype: $\widehat{\jmath}$, China, Yunnan, Daluo, NWAFU. Paratypes: $3 \hat{\jmath} \hat{\jmath} 4++$, same data as holotype, NWAFU.

Miridae

Fulvius yunnanicus Gorczyca, Wolski \& Taszakowski, 2020

Gorczyca et al. 2020. Bonn zoological Bulletin, 69 (1): 123-130

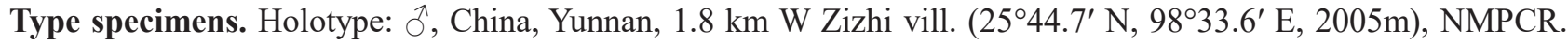
Paratype: 1 , , same data as for holotype, NMPCR.

Pseudococcidae

Atrococcus rushuiensis Zhang, 2020 
刘童神, 陈静, 姜立云, 乔格侠 (2021) 中国半翅目等29目昆虫2020年新分类单元. 生物多样性, 29, 1050-1057. http://www.biodiversity-science.net/CN/10.17520/biods.2021200.

Zhang et al. 2020. ZooKeys, 950: 33-40

Type specimens. Holotype: + , China, Jiangxi, Fuzhou City, Rushui Forest Park $\left(27^{\circ} 58^{\prime} \mathrm{N}, 116^{\circ} 22^{\prime} \mathrm{E}\right)$, JAU. Paratypes: 8 우, same data as holotype, JAU.

Kermicus huizhouensis Wu \& Huang, 2020

Wu et al. 2020. Zootaxa, 4859 (3): 440-450

Type specimens. Holotype: adult female, China, Guangdong, Huizhou, Huiyang, Qianfeng, BFUC. Paratypes: 5 adult females, 6 first-instar nymphs, 4 second-instar female nymphs, all same data as holotype, BFUC; 2 adult females, 4 first-instar nymphs, same location as holotype, GEEP.

Anomoneura taiwanica Cho \& Liao, 2020

Cho et al. 2020. ZooKeys, 917: 117-126

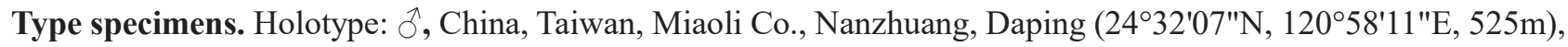
NCHU. Paratypes: $108 \precsim 103+$, China, Taiwan, 6 immatures, same data as for holotype, NCHU, TNMNS,

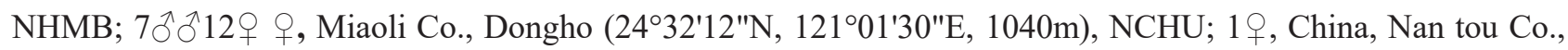
Huisun forest station, $\left(24^{\circ} 05^{\prime} 23^{\prime \prime} \mathrm{N}, 121^{\circ} 01^{\prime} 50^{\prime \prime} \mathrm{E}, 694 \mathrm{~m}\right), \mathrm{NCHU} ; 10{ }^{\circledR} 33$ 우, 14 immatures, same locality as for preceding, NCHU.

Chenicoris Chen \& Cai, 2020

Reduviidae

Chenicoris dilatatus Chen \& Cai, 2020

Chen et al. 2020. European Journal of Entomology, 117, 252-259

Type specimens. Holotype: $\widehat{\partial}$, China, Guangxi, Jinxiu, Mt. Dayaoshan, Luoyingou (1200m), CAU. Paratypes: 1 ,

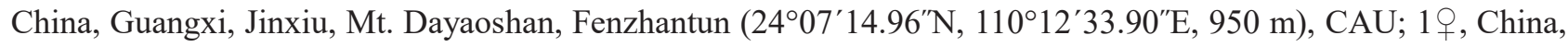

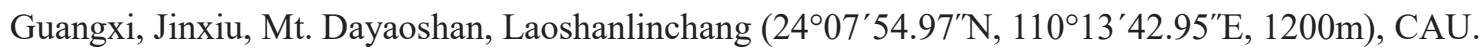

Chinemesa chinensis Chen, Li \& Cai, 2020

Chen et al. 2020. Annales de la Société entomologique de France (N.S.)

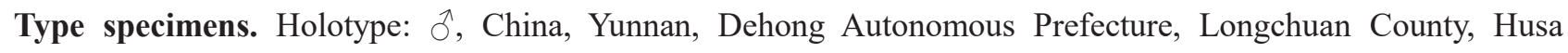
$\left(24^{\circ} 27^{\prime} 36^{\prime \prime} \mathrm{N} 97^{\circ} 53^{\prime} 24^{\prime \prime} \mathrm{E}, 1700 \mathrm{~m}\right), \mathrm{CAU}$. Paratypes: $4 \hat{\sigma}^{\hat{\partial}}$, same locality and collector as holotype, CAU.

Trioza turouguei Tung, Liao, Burckhardt \& Yang, 2020

Tung et al. 2020. ZooKeys, 958: 91-106

Type specimens. Holotype: ${ }^{\top}$, China, Taiwan, Taichung City, Shalien Lane (2411'20"N, $\left.120^{\circ} 55^{\prime} 06^{\prime \prime} \mathrm{E}\right)$, NCHU. Paratypes: $15 \delta^{\lambda} 17 q$ 우, China, Taiwan, Taichung City, Upper Kukuan; $1 \oint^{\lambda}, 1 q, 6$ immatures, Nantou Co., Hui-Sun Forest Station $\left(24^{\circ} 05^{\prime} 24^{\prime \prime} \mathrm{N}, 121^{\circ} 02^{\prime} 03^{\prime \prime} \mathrm{E}\right)$, NCHU, NHMB, TNMNS.

Veliidae

Perittopus anthracinus Ye \& $\mathrm{Bu}, 2020$

Ye et al. 2020. Zootaxa, 4858 (3): 417-426

Type specimens. Holotype: + , apterous, China, Yunnan, Honghe, Jinping County, Adebo village (22 $54^{\prime} \mathrm{N}$, $\left.103^{\circ} 13^{\prime} \mathrm{E}\right)$, NKU. Paratypes: $1{ }^{\top} 1$, , apterous, same data as holotype, NKU. 
刘童神, 陈静, 姜立云, 乔格侠 (2021) 中国半翅目等29目昆虫2020年新分类单元. 生物多样性, 29, 1050-1057. http://www.biodiversity-science.net/CN/10.17520/biods.2021200.

\section{螳螂目 Mantodea}

Eremiaphilidae

Didymocorypha libaii Wu \& Liu, 2020

Wu \& Liu. 2020. ZooKeys, 922: 51-63

Type specimens. Holotype: ${ }^{\lambda}$, China, Tibet, Gyirong County $(28.404 \mathrm{~N}, 85.332 \mathrm{E}, 3300 \mathrm{~m})$, IZCAS. Paratypes: $4 \lesssim \widehat{\delta} 6$ 우 , China, Tibet, Gyirong County (28.397N, 85.351E, 2800-3300 m), IZCAS; $3 \overbrace{}^{\jmath} 3$ 우우, PWC ; 1 , , CJZ; 1 , , Tibet, Gyirong County (28.363N, 85.339E, 2672 m), PWC.

\section{Mantidae 螳科}

Hierodula maculata Wang, Zhou \& Zhang, 2020 污斑斧螳

Wang et al. 2020. Entomotaxonomia, 42(2): 81-100

Type specimens. Holotype: $\widehat{\partial}$, China, Guangdong, Chebaling (350 m), SEMCAS. Paratypes: China, $1 \overbrace{}^{\lambda}$, Sichuan, Leshan city, SEMCAS; 2 + , Sichuan, Leshan city, SEMCAS.

Hierodula macrodentata Wang, Zhou \& Zhang, 2020 巨齿斧螳

Wang et al. 2020. Entomotaxonomia, 42(2): 81-100

Type specimens. Holotype: $\hat{\jmath}$, China, Yunnan, Jinghong Sanchahe, SEMCAS. Paratypes: $1 \precsim 1 \uparrow$, same as holotype, SEMCAS.

Hierodula pistillinota Wang, Zhou \& Zhang, 2020 杵胸斧螳

Wang et al. 2020. Entomotaxonomia, 42(2): 81-100

Type specimens. Holotype: $\hat{\sigma}$, China, Yunnan, Jinghong Rezuosuo), SEMCAS; Paratype: $1 \hat{\delta}$, China, Yunnan, Xishuangbanna (545 m), NWAFU.

\section{长翅目 Mecoptera}

Bittacidae

Bittacus acutus Zhang, Du \& Hua, 2020

Zhang et al. 2020. Zootaxa, 4718 (3): 381-390

Type specimens. Holotype: ${ }^{\lambda}$, China, Guangxi, Mao'ershan Nature Reserve $\left(25^{\circ} 53^{\prime} 25^{\prime \prime} \mathrm{N}, 110^{\circ} 29^{\prime} 08^{\prime \prime} \mathrm{E}, 1100 \mathrm{~m}\right)$, NWAFU. Paratype: 1 , same data as for the holotype, NWAFU.

Bittacus longilobus Zhang, Du \& Hua, 2020

Zhang et al. 2020. Zootaxa, 4718 (3): 381-390

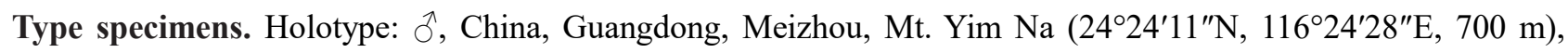
NWAFU. Paratypes: $5 \overbrace{}^{\lambda} 5$ 우의, same data as for the holotype, NWAFU.

Bittacus shaoguanensis Zhang, Du \& Hua, 2020

Zhang et al. 2020. Zootaxa, 4718 (3): 381-390

Type specimens. Holotype: ${ }^{\lambda}$, China, Guangdong, Shaoguan, Tsao Kok Wan $\left(24^{\circ} 43^{\prime} 35^{\prime \prime} \mathrm{N}, 113^{\circ} 53^{\prime} 06^{\prime \prime} \mathrm{E}, 300 \mathrm{~m}\right)$, NWAFU. Paratypes: $14 \delta^{\lambda} 8$ 우, same data as for the holotype, NWAFU.

\section{Panorpidae}

Dicerapanorpa bifurcata $\mathrm{Hu} \& \mathrm{Hua}, 2020$

Hu \& Hua. 2020. European Journal of Taxonomy, 711: 1-13

Type specimens. Holotype: $\partial^{\curvearrowright}$, China, Sichuan, Pingwu County, Wanglang Nature Reserve $\left(32.92^{\circ} \mathrm{N}, 104.16^{\circ} \mathrm{E}\right.$, 
刘童神, 陈静, 姜立云, 乔格侠 (2021) 中国半翅目等29目昆虫2020年新分类单元. 生物多样性, 29, 1050-1057. http://www.biodiversity-science.net/CN/10.17520/biods.2021200.

$2500 \mathrm{~m})$, NWAU. Paratypes: $2 \widehat{\delta} 14+$ 우, China, Sichuan, same data as for the holotype, NWAU; $1 \hat{\jmath}$, Maoxian County, Tudiling $\left(31.71^{\circ} \mathrm{N}, 103.92^{\circ} \mathrm{E}, 2500 \mathrm{~m}\right)$, NWAU.

Dicerapanorpa zhengkuni Hu \& Hua, 2020

Hu \& Hua. 2020. European Journal of Taxonomy, 711: 1-13

Type specimens. Holotype: $\partial^{\lambda}$, China, Guizhou, Jiangkou County, Fanjinshan Nature Reserve $\left(27.91^{\circ} \mathrm{N}, 108.65^{\circ}\right.$

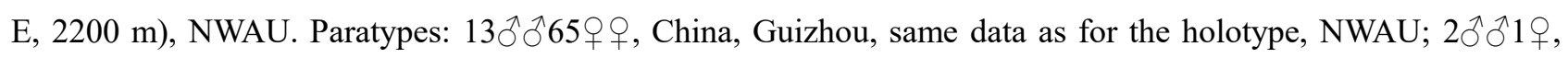
Leishan County, Leigongshan Nature Reserve (26.37 N, $\left.108.17^{\circ} \mathrm{E}, 1600 \mathrm{~m}\right)$, NWAU.

Cerapanorpa minshana Gao, Li \& Hua, 2020

Gao et al. 2020. ZooKeys, 971: 17-30

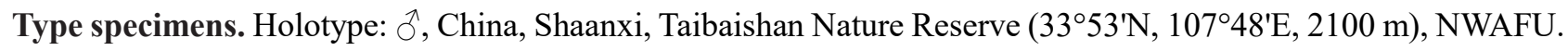

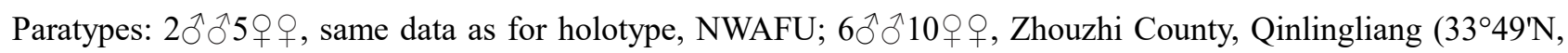

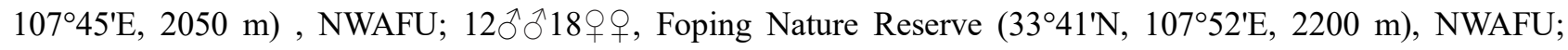

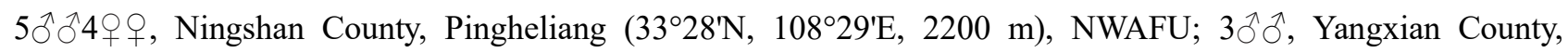
Changqing Nature Reserve ( $\left.33^{\circ} 42^{\prime} \mathrm{N}, 107^{\circ} 32^{\prime} \mathrm{E}, 2400 \mathrm{~m}\right)$, NWAFU.

Cerapanorpa qinlingensis Gao, Li \& Hua, 2020

Gao et al. 2020. ZooKeys, 971: 17-30

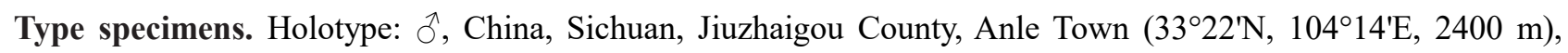

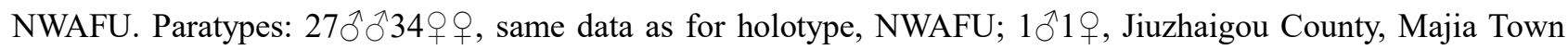

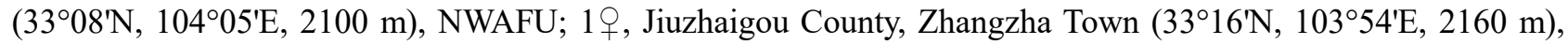
NWAFU; $18 \overbrace{}^{\top} 22$ 우, Gansu, Wenxian County, Gaoloushan $\left(33^{\circ} 04^{\prime} \mathrm{N}, 104^{\circ} 42^{\prime} \mathrm{E}, 2200 \mathrm{~m}\right)$, NWAFU.

Panorpa diqingensis Li \& Hua, 2020

Li \& Hua. 2020. Journal of Asia-Pacific Entomology, 23: 138-145

Type specimens. Holotype: $\widehat{\partial}$, China, Yunnan, Diqing, Shangri-La (2600-2700 m). Paratypes: $33 \AA \widehat{\partial} 25 q \propto$, same data as for the holotype, NWAFU.

Panorpa dispergens Li \& Hua, 2020

Li \& Hua. 2020. Journal of Asia-Pacific Entomology, 23: 138-145

Type specimens. Holotype: $\delta^{\lambda}$, China, Yunnan, Diqing, Shangri-La $\left(27.37^{\circ} \mathrm{N}, 100.13^{\circ} \mathrm{E}, 2700 \mathrm{~m}\right)$, NWAFU. Paratypes: $1 \delta^{\lambda} 3$ 우, same data as holotype, NWAFU; $13 \delta^{\Uparrow} 12$ 웅, Diqing, ShangriLa (2600-2700 m), NWAFU.

\section{脉翅目 Neuroptera}

Berothidae

Berotha incurvata Li, H. Aspock, U. Aspock \& Liu, 2020

Li et al. 2020. Zootaxa, 4890 (4): 509-520

Type specimens. Holotype: $\mathcal{\partial}$, China, Yunnan, Puer City, Simao District, Dachun River [大椿河] (1750 m), CAU. Paratypes: $10^{\lambda} 1{ }^{\lambda}$, China, Yunnan, Yuxi City, Yuanjiang County, Mt. Jiangdongmianshan [元江江东面山] (900 m), CAU. 
刘童神, 陈静, 姜立云, 乔格侠 (2021) 中国半翅目等29目昆虫2020年新分类单元. 生物多样性, 29, 1050-1057. http://www.biodiversity-science.net/CN/10.17520/biods.2021200.

Ankylopteryx yangi Ma, Yang \& Liu, 2020

Ma et al. 2020. ZooKeys, 906: 41-71

Type specimens. Holotype: ${ }^{\lambda}$, China, Guizhou, Libo, Maolan, Limingguan, CAU. Paratypes: 1 , China, Guizhou, Libo, Maolan Limingguan, CAU; 1, China, Taiwan, Pingtung, Lilungshan, CAU.

Austrochrysa angusta Ma, Yang \& Liu, 2020

Ma et al. 2020. Zootaxa, 4822 (1): 101-112

Type specimens. Holotype: $\widehat{\partial}$, China, Yunnan, Xishuangbanna (西双版纳), Menglun (预仑), No.55 Experimental site (780 m), CAU. Paratypes: $20 \hat{\jmath} \widehat{\jmath} 17$ 우, China, Yunnan, Xishuangbanna (西双版纳), Menglun (预仑), No.55 Experimental site (780 m), CAU; $3 \hat{\jmath} \hat{\jmath} 1$ 운, China, Yunnan, Xishuangbanna (西双版纳), Menglun (预仑), No.55 Experimental site (780 m), CAU.

Austrochrysa lihongyui Ma, Yang \& Liu, 2020

Ma et al. 2020. Zootaxa, 4822 (1): 101-112

Type specimens. Holotype: $\widehat{\partial}$, China, Tibet, Mêdog (墨脱), Miri vill. (810 m), CAU. Paratype: $1 \widehat{\jmath}$, China, Xizang, Mêdog (墨脱), Miri vill. (810 m), CAU.

\section{Dilaridae}

Dilar muliensis Li \& Liu, 2020

Li \& Liu. 2020. Journal of Asia-Pacific Entomology, 23: 915-922

Type specimens. Holotype: ${ }^{\top}$, China, Sichuan, Liangshan State, Muli County, Boke Village (N28 $06^{\prime} 11^{\prime \prime}$

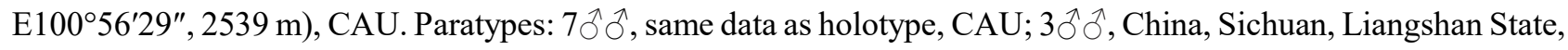
Muli County, Keer Village, Songjiagou (N2803'43" E10101'48", 2539 m), CAU.

Dilar punctulosus Li \& Liu, 2020

Li \& Liu. 2020. Journal of Asia-Pacific Entomology, 23: 915-922

Type specimen. Holotype: $\widehat{\partial}$, China, Sichuan, Liangshan State, Muli County, Shawan Town, Nawa Village, near Wujia River (N28 $15^{\prime} 24^{\prime \prime}$ E100 $54^{\prime} 12^{\prime \prime}, 2619$ m), CAU.

Rapisma weixiense Liu, 2020

Ithonidae

Liu. 2020. Zootaxa, 4763 (3): 439-443

Type specimen. Holotype: đ̊, China, Yunnan, Weixi, Samage (27³2’37.40”N 99²1’15.74’'E, 2182 m), CAU.

\section{Mantispidae}

Allomantispa coniprocessa $\mathrm{Li}, \mathrm{Wu}, \mathrm{Ohl} \& \mathrm{Liu}, 2020$

Li \& Liu. 2020. Journal of Asia-Pacific Entomology, 988-1002

Type specimens. Holotype: $\widehat{\partial}$, China, Yunnan, Xishuangbanna, Mengla County (1100 m), CAU. Paratypes: 1 , China, Yunnan, Xishuangbanna, Mengla County (1100 m), IZCAS; 1ðð, China, Yunnan, Honghe, Lvchun County, Huanglianshan National Nature Reserve (1450 m), CAU; 1ठ, China, Yunnan, Honghe, Lvchun County, Huanglianshan National Nature Reserve (1450 m), IZCAS; 1 , China, Yunnan, Xishuangbanna, Mengla County (1100 m), CAU; 1q, China, Yunnan, Honghe, Lvchun County, Huanglianshan National Nature Reserve (1450 m), PCW. 
刘童神, 陈静, 姜立云, 乔格侠 (2021) 中国半翅目等29目昆虫2020年新分类单元. 生物多样性, 29, 1050-1057. http://www.biodiversity-science.net/CN/10.17520/biods.2021200.

\section{Calopterygidae}

Caliphaea hermannkunzi Zhang \& Hämäläinen, 2020

Zhang \& Hämäläinen. 2020. Zootaxa, 4895 (1): 103-110

Type specimens. Holotype: $\partial^{\wedge}$, China, Yunnan, Lufeng County, Chuxiong City, Gaofeng village, $\left(25^{\circ} 18^{\prime} 43^{\prime \prime} \mathrm{N}\right.$,

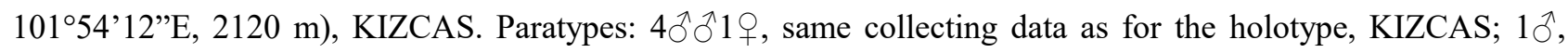
RMNH, KIZCAS.

\section{Platycnemididae}

Coeliccia tongbiguan Dow \& Zhang, 2020

Dow \& Zhang. 2020. Zootaxa, 4767 (3): 492-494

Type specimens. Holotype: $\widehat{\jmath}$, China, Yunnan, Yingjiang County, Dehong Dai \& Jingpo Autonomous Prefecture,

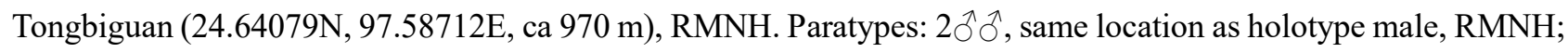
$2 \hat{\delta} \hat{\partial}$, same location as holotype male, RMNH.

Coeliccia yunnanensis Dow \& Zhang, 2020

Dow \& Zhang. 2020. Zootaxa, 4767 (3): 492-494

Type specimens. Holotype: $\hat{\sigma}$, China, Yunnan, Yingjiang County, Dehong Dai \& Jingpo Autonomous Prefecture,Tongbiguan (24.61522N, 97.58558E, ca 1245 m), RMNH. Paratypes: $1{ }^{\Uparrow} 1$ \% , data as holotype, RMNH;

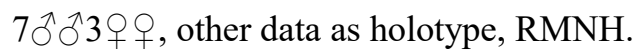

\section{直翅目 Orthoptera}

Acrididae

Euthystiroides heilongjiangensis Li, Wen \& Zhi, 2020

Li et al. 2020. Zootaxa, 4767 (3): 492-494

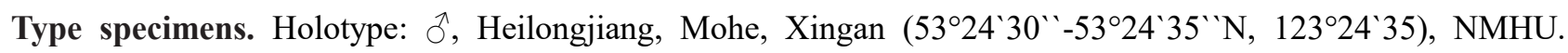

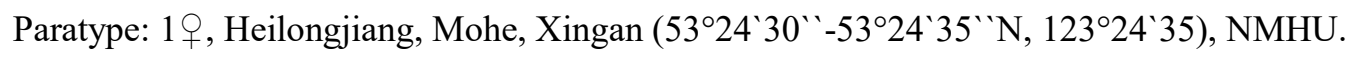

Mongolotettix moheensis Zhang, Lv \& Yin, 2020

Zhang et al. 2020. Zootaxa, 4759 (1): 147-150

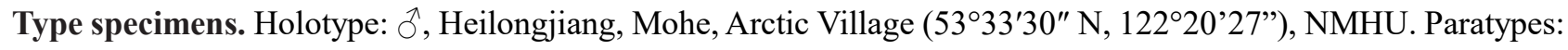

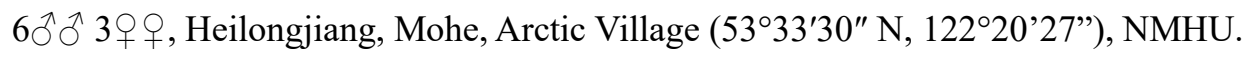

\section{Melanabropsis Wang \& Liu, 2020 乌黯虫属}

Melanabropsis tianmuica Wang \& Liu, 2020 天目乌黯蚉

Wang \& Liu. 2020. Zootaxa, 4809 (3): 509-520

Type specimens. Holotype: $\widehat{\partial}$, Zhejiang, west Tianmu Mountain, Lin'an City (1100m), SEMCAS. Paratypes: $1 \overbrace{}^{\lambda}$, Zhejiang, west Tianmu Mountain, Lin'an City (1132m), SEMCAS; $1 ð$, Zhejiang, Tianmu Mountain N. R., Lin'an City (1100 m), SEMCAS; $1 \hat{\jmath}$, Zhejiang, west Tianmu Mountain, (1100 m), SEMCAS; $1 \hat{\jmath}$, China, Guangxi, Guangfuding, Huaping N. R., Lin'gui County (1780 m), SEMCAS; 1ð̂, Guangxi, Mao'er Mountain (1600 m), SEMCAS; $2+q$, Zhejiang, west Tianmu Mountain (1100m), SEMCAS; $2+q$, Zhejiang, Laodian, west Tianmu Mountain (1100m), SEMCAS; 1 \%, west Tianmu Mountain, Zhejiang (1100m), SEMCAS; 1ㅇ, Zhejiang, west Tianmu Mountain (1200 m), SEMCAS; 1q, Zhejiang, west Tianmu Mountain, Lin'an City (987 1132 m), SEMCAS; 1, Zhejiang, Tianmu Mountain N. R., (1402 1500m), SEMCAS. 
刘童神, 陈静, 姜立云, 乔格侠 (2021) 中国半翅目等29目昆虫2020年新分类单元. 生物多样性, 29, 1050-1057. http://www.biodiversity-science.net/CN/10.17520/biods.2021200.

\section{Batrachididae}

Saussurella yunnanensis Mao, Han \& Li, 2020

Han et al. 2020. Entomotaxonomia, 42(1): 6-11

Type specimens. Holotype: $\stackrel{+}{+}$, Yunnan, Yingjiang $\left(24^{\circ} 40^{\prime} \mathrm{N}, 97^{\circ} 35^{\prime} \mathrm{E}, 674 \mathrm{~m}\right)$, BMDU. Paratype: $1 \partial^{\prime}$, data same as holotype, BMDU.

\section{Catantopidae}

Lemba wushanensis Yin, Shen \& Yin, 2020

Yin et al. 2020. Zootaxa, 4894 (2): 287-291

Type specimens. Holotype: $\widehat{\jmath}$, Chongqing, Wushan, NPIBCAS. Paratypes: $1 \stackrel{\uparrow}{ }$, Chongqing, Wushan, NPIBCAS.

$$
\text { Gryllacrididae }
$$

Eremus lincangensis Yang, Jing \& Bian, 2020

Yang et al. 2020. Zootaxa, 4895 (1): 146-150

Type specimens. Holotype: $\hat{\jmath}$, China, Yunnan, Nangunhe National Nature Reserve, Wengding Village Mengjiao Town, Cangyuan Country, Lincang Prefecture $\left(23^{\circ} 16^{\prime} 39.1^{\prime \prime} \mathrm{N}, 99^{\circ} 11^{\prime} 21.4^{\prime \prime} \mathrm{N}, 1790 \mathrm{~m}\right)$, BMDU. Paratype: $1^{\jmath} \mathrm{O}^{\prime}$ China, Yunnan, Mohan, Mengla, BMDU.

Loxoblemmus rectilineus Ma \& Qiao, 2020

Gryllidae

Qiao et al. 2020. Zootaxa, 4732 (3): 481-487

Type specimens. Holotype: $\hat{\jmath}$, China, Yunnan, Xishuangbanna, botanical garden, SNNU. Paratypes: $\hat{\jmath}$, China, Yunnan, Mengla District, Wangtianshu, SNNU.

Mirigryllus He, 2020

Mirigryllu nigrus He, 2020

He et al. 2020. Zootaxa, 4869 (1): 112-120

Type specimens. Holotype: ふ̋, China, Zhejiang, Lin’an, Qingliangfeng Mt. (N 3006'39" E 11854'01", 920m),

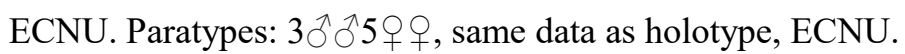

Tarbinskiellus sororius Wang, Zhang \& Liu, 2020

Wang et al. 2020. Zootaxa, 4729 (4): 575-581

Type specimens. Holotype: $\widehat{\jmath}$, China, Yunnan, Lvchun, Kucong, Luoshi, NMHU. Paratypes: $2 \hat{\jmath} 2 \propto \uparrow$, same data as the holotype, NMHU; $2 \hat{\jmath}$, China, Yunnan, Lvchun, Yakou, NMHU.

Mogoplistidae

Ornebius yunnanensis Wang, Zhang \& Liu, 2020

Wang et al. 2020. Zootaxa, 4729 (4): 575-581

Type specimen. Holotype: $\lesssim$, China, Yunnan, Malipo, Mt. Laojun, NMHU.

Haplotropis heiheensis Zhang, Li \& Yin, 2020

Pamphagidae

Zhang et al. 2020. Zootaxa, 4742 (2): 392-394 
刘童神, 陈静, 姜立云, 乔格侠 (2021) 中国半翅目等29目昆虫2020年新分类单元. 生物多样性, 29, 1050-1057. http://www.biodiversity-science.net/CN/10.17520/biods.2021200.

Type specimens. Holotype: $\partial^{\lambda}$, China, Heilongjiang, Heihe City $\left(50^{\circ} 19^{\prime} \mathrm{N}, 127^{\circ} 17^{\prime} \mathrm{E}, 266 \mathrm{~m}\right)$, SAU. Paratypes: $1 \delta^{\top} 6$ 우우, China, $\left(50^{\circ} 19^{\prime} \mathrm{N}, 127^{\circ} 17^{\prime} \mathrm{E}, 266 \mathrm{~m}\right)$, SAU.

Sulcohumpacris wuanensis Li, Zhi \& Yin, 2020

Li et al. 2020. Zootaxa, 4869 (1): 146-148

Type specimens. Holotype: ${ }^{\top}$, Paratype: 1 울, China, Hebei, Wuan, $36^{\circ} 44^{\prime} \mathrm{N}, 113^{\circ} 59^{\prime} \mathrm{E}$, NMHU.

Sulcotropis xiaowutaiensis Li, Zhang \& Yin, 2020

Li et al. 2020. Zootaxa, 4802 (3): 541-555

Type specimens. Holotype: $\widehat{\partial}$, Paratypes: $1{ }^{\Uparrow} 2$ 우, China, Hebei, Xiaowutai mountain, NMHU.

Rhaphidophoridae

Gymnaetoides polaoensis Feng, Huang \& Luo, 2020

Feng et al. 2020. Zootaxa, 4885 (3): 447-450

Type specimens. Holotype: $\widehat{\delta}$, China, Guizhou, Polao Cave, Zhenfeng County, Southwest Guizhou Autonomous Prefecture. Paratype: $1{ }^{\lambda}$, same data as the holotype. GUGC.

Microtachycines trispinosus Qin \& Li, 2020

Qin et al. 2020. Zootaxa, 4801 (3): 570-576

Type specimens. Holotype: $\widehat{\partial}$, China, Jiangxi, Sanqingshan, Tiyunling-Xianrenzhilu (1240 1400m). Paratype: $1{ }^{\lambda}$, China, Jiangxi, Sanqingshan, Xihai'an-Sanqingfudi-Yangguanghai'an (1500m), SEMCAS.

Tachycines shuangcha Feng, Huang \& Luo, 2020

Feng et al. 2020. Zootaxa, 4820 (3): 563-571

Type specimens. Holotype: ${ }^{\lambda}$, China, Guizhou, Shuangcha Cave, Mayanghe National Nature Reserve, Yanhe Tujia Autonomous County, Tongren City, GUGC. Paratypes: $7 \hat{\jmath}$ $50+$, same data as the holotype, GUGC.

Tachycines tongrenus Feng, Huang \& Luo, 2020

Feng et al. 2020. Zootaxa, 4820 (3): 563-571

Type specimens. Holotype: ${ }^{\lambda}$, China, Guizhou, Shuangcha Cave, Mayanghe National Nature Reserve, Yanhe Tujia Autonomous County, Tongren City, Guizhou, GUGC. Paratypes: $12 \overbrace{}^{\lambda} 39+9$, same data as the holotype, GUGC.

Tachycines zaoshu Feng, Huang \& Luo, 2020

Feng et al. 2020. Zootaxa, 4820 (3): 563-571

Type specimens. Holotype: $\widehat{\delta}$, China, Guizhou, Zaoshu Cave, Mayanghe National Nature Reserve, Yanhe Tujia

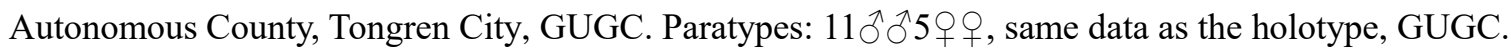

Tachycines trapezialis Zhou \& Yang, 2020

Zhou \& Yang. 2020. ZooKeys, 937: 21-29

Type specimens. Holotype: ${ }^{\top}$, China, Guizhou, Diaosiyan Cave, Ziyun County, $\left(25^{\circ} 35.06^{\prime}\right.$ N, $106^{\circ} 12.32^{\prime}$ E, 1110 1120 m), GZNU. Paratypes: 1\%, same data as holotype, GZNU.

Tachycines bifolius, Zhu, Chen \& Shi, 2020

Zhu et al. 2020. Zootaxa, 4809 (1): 071-094 
刘童神, 陈静, 姜立云, 乔格侠 (2021) 中国半翅目等29目昆虫2020年新分类单元. 生物多样性, 29, 1050-1057. http://www.biodiversity-science.net/CN/10.17520/biods.2021200.

Type specimens. Holotype: $\curvearrowright$ China, Guizhou, Shahe Cave, Yunihe, Panzhou City. Paratypes: $1 \hat{\jmath} 20+q$, same collection data as for holotype, NMHU, GZIB.

Tachycines latiliconcavus Zhu, Chen \& Shi, 2020

Zhu et al. 2020. Zootaxa, 4809 (1): 071-094

Type specimens. Holotype: $\widehat{\jmath}$, China, Sichuan, Longmen Cave, Longmen Village, Lushan County. Paratypes: $3 \widehat{\jmath} 1$, , same collection data as for holotype, NMHU, GZIB.

Tachycines liboensis Zhu, Chen \& Shi, 2020

Zhu et al. 2020. Zootaxa, 4809 (1): 071-094

Type specimen. Holotype: đ̊, China, Guizhou, Maan Cave, Banzhai, Libo County, NMHU, GZIB.

Tachycines nulliscleritus Zhu, Chen \& Shi, 2020

Zhu et al. 2020. Zootaxa, 4809 (1): 071-094

Type specimens. Holotype: $\widehat{\jmath}$, China, Hunan, Jinji Cave, Nanjiaping, Huangyan Village. Paratype: 19 , same collection data as for holotype. NMHU, GZIB.

Tachycines paradoxus Zhu, Chen \& Shi, 2020

Zhu et al. 2020. Zootaxa, 4809 (1): 071-094

Type specimens. Holotype: $\widehat{\jmath}$, China, Guizhou, Cave, Pogang Village, Zhengtun Town, Xingyi City. Paratypes: $3 \widehat{\jmath} 1$, , same collection data as for holotype, NMHU, GZIB.

Tachycines taenus Zhu, Chen \& Shi, 2020

Zhu et al. 2020. Zootaxa, 4809 (1): 071-094

Type specimens. Holotype: $\hat{\sigma}$, China, Guangxi, Gan Cave, Quishang Tun, Xinyan Village, Donglan County. Paratype: 1 , same collection data as for holotype, NMHU, GZIB.

Tachycines tuberus Zhu, Chen \& Shi, 2020

Zhu et al. 2020. Zootaxa, 4809 (1): 071-094

Type specimens. Holotype: $\widehat{\jmath}$, China, Guizhou, Zhuanwan Cave, Sanglang Town, Wangmo County. Paratypes: $1 \precsim 2$ 우, same collection data as for holotype, NMHU, GZIB.

Tachycines umbellus Zhu, Chen \& Shi, 2020

Zhu et al. 2020. Zootaxa, 4809 (1): 071-094

Type specimens. Holotype: $\curvearrowright$, China, Hunan, Xianhua Cave, Suoxiyu, Zhangjiajie City. Paratypes: $1 \delta 2 q q$, same collection data as for holotype, NMHU, GZIB.

Paradiestrammena trigona Zhu, Lu \& Shi, 2020

Zhu et al. 2020. Journal of Natural History, 54:19-20

Type specimens. Holotype: $\hat{\jmath}$, China, Yunnan, Benlonggong, Yingjiang, NMHU. Paratypes: $1 \hat{\jmath}$, China, Yunnan, Songke, Yingjiang; $2 \uparrow q$, China, Yunnan, Tongbiguan, Yingjiang; $2 \lesssim \nwarrow 1 q$, China, Yunnan, Tongbiguan, Yingjiang, Yunnan, NMHU. 
刘童神, 陈静, 姜立云, 乔格侠 (2021) 中国半翅目等29目昆虫2020年新分类单元. 生物多样性, 29, 1050-1057. http://www.biodiversity-science.net/CN/10.17520/biods.2021200.

Zhu et al. 2020. Journal of Natural History, 54:19-20

Type specimen. Holotype: $\widehat{\jmath}$, China, Yunnan, Xima, Yingiiang, NMHU.

Tetrigidae 蚱科

Formosatettix leigongshanensis Zha \& Ding, 2020

Zha et al. 2020. ZooKeys, 936: 61-76

Type specimens. Holotype: + , China, Guizhou, Leishan County, Leigong-shan Mt., (26 $22^{\prime} 45.6^{\prime \prime}$ N, 108 11'42.83" E, 1460 m), HNU. Paratypes: $15 \overbrace{}^{\lambda}{ }^{\wedge} 6$ 우 , China, Guizhou, Leigong-shan Mt. (1400-1600 m), HNU.

Formosatettix wulongensis Zha \& Ding, 2020

Zha et al. 2020. ZooKeys, 936: 61-76

Type specimens. Holotype: + , China, Chongqing Autonomous Region, Wulong County, $\left(29^{\circ} 20^{\prime} 32.27^{\prime \prime} \mathrm{N}\right.$, $107^{\circ} 45^{\prime} 23.35^{\prime \prime}$ E, $\left.470 \mathrm{~m}\right)$, HNU. Paratypes: $1 \delta^{\wedge} 2$ 우, same data as holotype, HNU.

Hainantettix Deng, 2020

Hainantettix strictivertex Deng, 2020

Zhang et al. 2020. Zootaxa, 4809 (3): 547-559

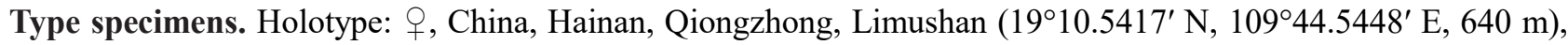
EMHU. Paratypes: China, Hainan: $1 \jmath^{\Uparrow} 1$, same data, type locality, EMHU; $1 \diamond^{\Uparrow} 1$, , China, Hainan, Lingshui,

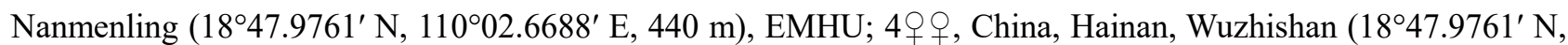
$110^{\circ} 02.6688^{\prime}$ E, 440 m), EMHU; 1ㅇ, China, Hainan, diaoluoshan, Nanmenling $\left(18^{\circ} 7.5598^{\prime} \mathrm{N}, 109^{\circ} 8.5162^{\prime} \mathrm{E}, 500\right.$

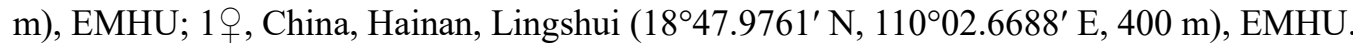

Lamellitettigodes diversifemoris $\mathrm{Lu} \&$ Zha, 2020

Liu \& Zha. 2020. Zootaxa, 4851 (2): 338-348

Type specimens. Holotype: + , China, Hainan, Ledong County, Jianfengling National Forest Park $\left(18^{\circ} 44^{\prime} 10.07^{\prime \prime} \mathrm{N}\right.$, $108^{\circ} 52^{\prime} 6.86^{\prime \prime}$ E, $\left.830 \mathrm{~m}\right)$, HNU. Paratypes: $2{ }^{\wedge}{ }^{\top} 2$ 2우 , (750-850 m), other data same as holotype, HNU.

Macromotettixoides daiyunshanensis Deng, 2020

Wei. 2020. Zootaxa, 4852 (1): 041-060

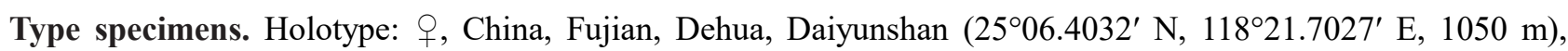
EMHU. Paratypes: $4 \hat{\jmath} 6$ 우우, same data, EMHU.

Macromotettixoides curvicarina Deng, 2020

Wei. 2020. Zootaxa, 4852 (1): 041-060

Type specimens. Holotype: + , China, Zhejiang, Longquan, Fengyangshan $\quad\left(27^{\circ} 52.5596^{\prime} \mathrm{N}, 119^{\circ} 10.8584^{\prime} \mathrm{E}\right.$,

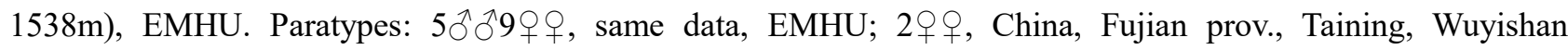
$\left(27^{\circ} 00.6084^{\prime} \mathrm{N}, 117^{\circ} 07.1163^{\prime} \mathrm{E}, 1513 \mathrm{~m}\right)$, EMHU.

Macromotettixoides convexa Deng, 2020

Wei. 2020. Zootaxa, 4852 (1): 041-060

Type specimens. Holotype: 웅, China, Fujian, Shanghang, Meihuashan $\left(25.342921^{\circ} \mathrm{N}, 116.838556^{\circ} \mathrm{E}, 1348 \mathrm{~m}\right)$, EMHU. Paratypes: 3 q 9 , same data, EMHU. 
刘童神, 陈静, 姜立云, 乔格侠 (2021) 中国半翅目等29目昆虫2020年新分类单元. 生物多样性, 29, 1050-1057. http://www.biodiversity-science.net/CN/10.17520/biods.2021200.

Macromotettixoides shengtangshanensis Deng, 2020

Wei. 2020. Zootaxa, 4852 (1): 041-060

Type specimens. Holotype: + , China, Guangxi, Jinxiu, shengtangshan $\left(23^{\circ} 58.5999^{\prime} \mathrm{N}, 110^{\circ} 04.4041^{\prime} \mathrm{E}, 1200 \mathrm{~m}\right)$, EMHU. Paratypes: $2 \hat{\jmath} 8$ 우, same data, EMHU.

Macromotettixoides tuberculata Mao, Li \& Han, 2020

Mao et al. 2020. Zootaxa, 4718 (4): 562-572

Type specimens. Holotype: ㅇ, China, Yunnan, Ruili $\left(24^{\circ} 04.55^{\prime}\right.$ N, $97^{\circ} 48.16^{\prime}$ E, 1417 m), BMDU. Paratype: 1 , same data as holotype, BMDU.

Macromotettixoides truncata Mao, Li \& Han, 2020

Mao et al. 2020. Zootaxa, 4718 (4): 562-572

Type specimens. Holotype: , , China, Yunnan, Yingjiang ( $24^{\circ} 36.84^{\prime}$ N, 97³9.39’ E, 1336 m), BMDU. Paratypes: 14 우우 $10 \hat{\jmath} \widehat{\partial}$, same data as holotype, BMDU.

Macromotettixoides rugodorsalis Li \& Mao, 2020 皱背拟大磨蚱

$\mathrm{Li}$ et al. 2020. Entomotaxonomia, 42(2): 110-115

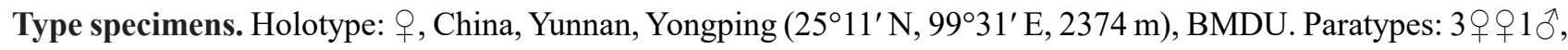
same locality as holotype, BMDU.

Pseudepitettix hainanensis Deng, 2020

Deng. 2020. Zootaxa, 4789 (2): 403-440

Type specimens. Holotype: $q$, China, Hainan, Baisha, Yinggeling $\left(19^{\circ} 02.3866^{\prime} \mathrm{N}, 109^{\circ} 34.1783^{\prime} \mathrm{E}, 350 \mathrm{~m}\right)$, EMHU.

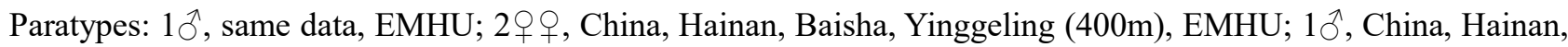
Baisha, Yinggeling (400m), EMHU.

Pseudepitettix strictivertex Deng, 2020

Deng. 2020. Zootaxa, 4789 (2): 403-440

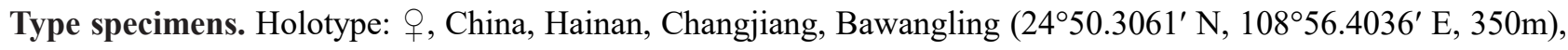
EMHU. Paratypes. $1 \overbrace{}^{\lambda}$, same data, type locality, EMHU; 2 우, China, Hainan, Baisha, Yinggeling (400m), EMHU; 10, China, Hainan, Baisha (Yinggeling), 400m, EMHU, NMHU.

Tuberfemurus convexa Deng, 2020

Deng. 2020. Zootaxa, 4789 (2): 403-440

Type specimens. Holotype: $q$, China, Zhejiang, Linan, Tianmushan, EMHU. Paratypes: $5 q q$, same data, EMHU. Tettigoniidae

Acosmetura longielata Wang \& Shi, 2020

Wang \& Shi. 2020. Journal of Orthoptera Research, 29(2): 115-120

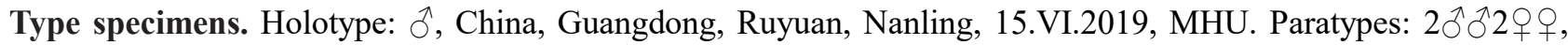
China, Guangdong, Ruyuan, Nanling, NMHU. 
刘童神, 陈静, 姜立云, 乔格侠 (2021) 中国半翅目等29目昆虫2020年新分类单元. 生物多样性, 29, 1050-1057. http://www.biodiversity-science.net/CN/10.17520/biods.2021200.

Wang et al. 2020. Zootaxa, 4802 (1): 182-188

Type specimens. Holotype: $\widehat{\jmath}$, China, Guizhou, Fangxiang, Leishan, NMHU. Paratypes: $2 \delta \hat{\jmath} 1 \uparrow$, China, Guizhou, Fangxiang, Leishan, NMHU; 1 , China, Guizhou, Fangxiang, Leishan.

Eoxizicus bifurcatus Liu, 2020

Jin et al. 2020. Zootaxa, 4772 (1): 001-053

Type specimen. Holotype: $\hat{\partial}$, China, Taiwan, Tsaoshan (200-300 m), BMH.

Pseudocosmetura yaoluopingensis Wang \& Shi, 2020

Wang \& Shi. 2020. Journal of Orthoptera Research, 29(2): 115-120

Type specimens. Holotype: $\widehat{\partial}$, China, Anhui, Yuexi, Yaoluoping, MHU. Paratypes: $1 \hat{\jmath}$, China, Anhui, Yuexi, Yaoluoping, NMHU; 1ㅇ, Anhui, Yuexi, Yaoluoping, NMHU.

Macroteratura inospina Chen, Cui \& Chang, 2020

Chen et al. 2020. Zootaxa, 4857 (1): 095-104

Type specimens. Holotype: $\stackrel{\lambda}{ }$, China, Yunnan, Tongbiguan, Yingjiang. Paratype: 19 , China, Yunnan, Tongbiguan, Yingjiang, NMHU.

Mecopoda crescendo Liu, 2020

Liu et al. 2020. Zootaxa, 4758 (2): 296-310

Type specimens. Holotype: đ̃, China, Yunnan, Mengla County, Wangtianshu Scenic Spot, (21.5998 N, 101.5869 E), IZCAS. Paratypes: $14 \hat{\delta} 1$, same data as in holotype, IZCAS; $6 \widehat{\delta} 2+q+$, China, Yunnan, Mengla County, Menglun Botanic Garden (21.9237 N, 101.2711), IZCAS; 5우, China, Yunnan, Menglun (795 m), IZCAS; 1 , China, Yunnan, Menglun, IZCAS.

Mecopoda himalaya Liu, 2020

Liu et al. 2020. Zootaxa, 4758 (2): 296-310

Type specimens. Holotype: $\widehat{O}$, China, Yunnan, Honghe City, Jinping County, Mengqiao Village, Mengping (474 m), IZCAS. Paratypes. 1 $\delta^{\lambda}$, China, Yunnan, Honghe City, Jinping County, Surburb of Jinping County (500 m), IZCAS.

Mecopoda tibetensis Liu, 2020

Liu et al. 2020. Zootaxa, 4758 (2): 296-310

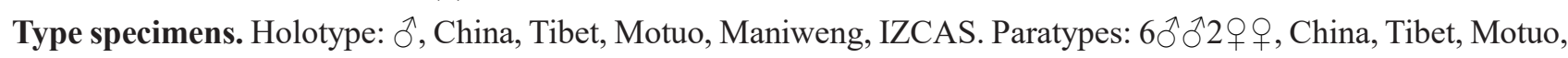
Urban District (1084 m), IZCAS; $10 \hat{\jmath}$; $1 \hat{\jmath}$, China, Tibet, Beibeng Village, IZCAS; $2 \hat{\jmath}$, China, Tibet, at light, 108K near Zamo Road, Qiaotou Hotel, IZCAS; $1 \hat{\jmath}$, China, Tibet, Maniweng, $2 \hat{\jmath}$, China, Tibet, Didong, IZCAS.

Mecopoda confracta Liu, 2020

Liu et al. 2020. Zootaxa, 4758 (2): 296-310

Type specimens. Holotype: đ̊ , China, Yunnan, Mengla County, Wangtianshu Scenic Spot, (21.5998 N 101.5869 E), IZCAS. Paratypes: $10 \hat{\jmath} \widehat{\jmath}$, same data as in holotype, IZCAS; , $6 \hat{\jmath} 1 \hat{1}$, China, Guangxi, Nanning City, Damingshan Scenic Spot, (23.3844 N 108.5507 E), IZCAS; $2 \hat{\jmath} \widehat{\partial}$, Longzhou City, Nonggang Nature Reserve, IZCAS; $1 \hat{\jmath}$, China Yunnan, Mengla County, Menglun Botanic Garden (21.9237 N 101.2711 E), IZCAS; $3 \hat{\jmath} \widehat{\partial}$, China, Yunnan, Wenshan County, Maguan Village, IZCAS; $1 \hat{\jmath}$, China, Yunnan, Jin Ping, IZCAS; $2 \hat{\jmath} \widehat{\partial} 2 q+q$, China, Yunnan, Ruili 
刘童神, 陈静, 姜立云, 乔格侠 (2021) 中国半翅目等29目昆虫2020年新分类单元. 生物多样性, 29, 1050-1057. http://www.biodiversity-science.net/CN/10.17520/biods.2021200.

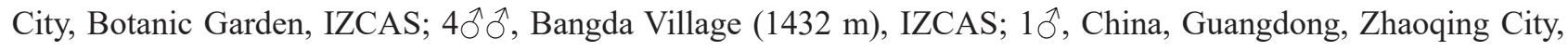

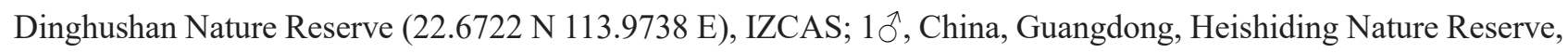
IZCAS.

Mecopoda synconfracta Liu, 2020

Liu et al. 2020. Zootaxa, 4758 (2): 296-310

Type specimens. Holotype: ${ }^{\lambda}$, China, Guangxi, Baise City, Tiandong City, Suburban District (23.6311 N, 107.1258

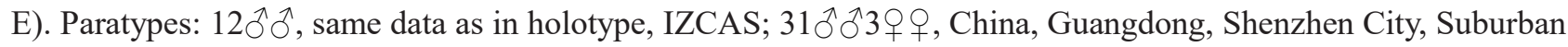

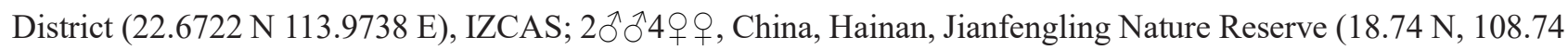
E), IZCAS; $25 \widehat{\jmath} 15$ 우, China, Hainan, Bawangling Nature Reserve, IZCAS; $2 \widehat{\jmath}$, China, Guangxi, Shaoping, IZCAS; 1 , China, Yunnan, Hekou City, Nanxi, IZCAS.

Mecopoda minor Liu, 2020

Liu et al. 2020. Zootaxa, 4758 (2): 296-310

Type specimens. Holotype: $q$ Korea, NHML. Paratypes: $1 \hat{\jmath}$, China, Hongkong; China, Anhui; $1 \hat{\jmath}$, China, Anhui,

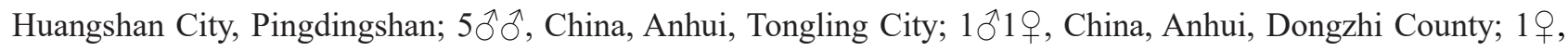
China, Fujian, Nanping City, Shangyang; 1 , China, Fujian, Shaowu County, Chengguan; $3 \hat{\jmath} \jmath 2 q q$, China, Fujian,

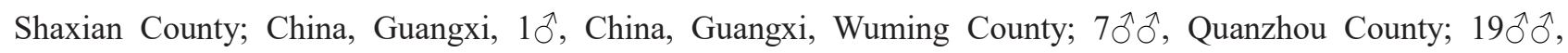

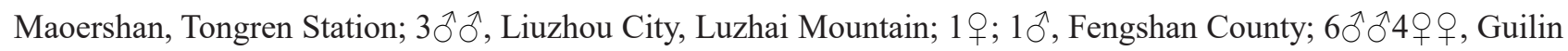
City; $3 \hat{\jmath} \widehat{\partial}$, Nanning City, Daming Mountain; China: Zhejiang: $10 \widehat{\jmath} 5$ 우, Jinhua City, Pujiang County; 1 , Zhoushan City; $1 \hat{\partial}$, Hangzhou City, Gushan. China, Hubei : $4 \hat{\partial} \widehat{\partial}$, Jingmen City, Queji Village; $6 \hat{\jmath}$, Huanggang City, Qichun County; China: Guizhou: 1 우 Meitan County; China, Hunan: $3 ㅇ+11 \hat{\jmath}$, Huarong County; $1 \hat{\delta}$, Cili

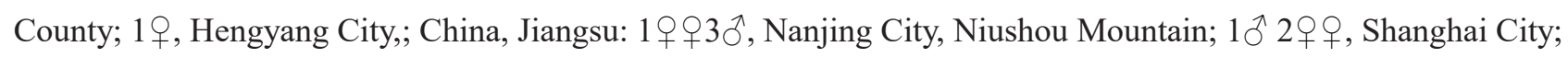
China, Jiangxi: $1 \hat{\jmath}$ Jiujiang County; $19+7 \hat{\jmath} \hat{\jmath}$, Ji'an City, Qingyuan Mountain; $1 \hat{\jmath}$, Lushan City, Luling Town,;

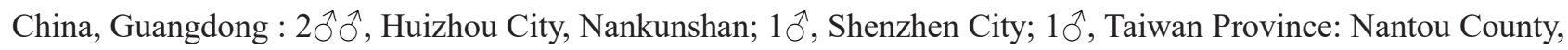
Huisun Forest Farm; China, Henan: $1 \overbrace{}^{\Uparrow}$, Xinyang City, Liankang Mountain; 1ㅇ, Sichuan ; $1{ }^{\Uparrow} 1$, , Chongqing City, IZCAS.

Mecopoda minor yunnana Liu, 2020

Liu et al. 2020. Zootaxa, 4758 (2): 296-310

Type specimens. Holotype: ${ }^{\top}$, China, Yunnan, Yuxi City, Cengjiang, Jiucheng Village (24.6668 N, 102.9463 E),

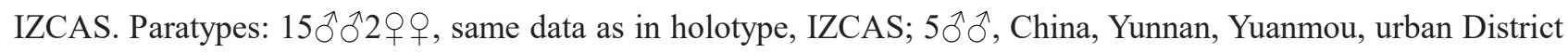
(25.7081 N 101.8732 E), IZCAS.

Nahlaksia hainanensis He \& Wang, 2020

He et al. 2020. Zootaxa, 4877 (1): 195-200

Type specimens. Holotype: $\delta^{\lambda}$, China, Hainan, Ledong, Jianfengling, ECNU. Paratypes: $1 \delta^{\Uparrow}, 1$ nymph, China, Hainan, Sanya, Baolong, ECNU; 3 đnymphs 4 q nymphs, China, Hainan, Changjiang, Bawangling, ECNU; 1 $1 \widehat{\jmath}$ nymph, China, Hainan, Wuzhishan, $400 \mathrm{~m} ; 1$ 웅, China, Hainan, Changjiang, Bawangling (460 1500 m), SEMCAS.

Neocosmetura Xin, Wang \& Shi, 2020 (新饰尾蚉属)

Neocosmetura torcha Xin, Wang \& Shi, 2020 (炬形新饰尾蚉)

Xin et al. 2020. Zootaxa, 4819 (2): 385-392 
刘童神, 陈静, 姜立云, 乔格侠 (2021) 中国半翅目等29目昆虫2020年新分类单元. 生物多样性, 29, 1050-1057. http://www.biodiversity-science.net/CN/10.17520/biods.2021200.

Type specimens. Holotype: $\hat{\alpha}$, China, Hunan, Jiuyishan, Ningyuan, NMHU. Paratypes: $3 \hat{\jmath} \widehat{\delta} 4 q \propto+$, China, Hunan, Jiuyishan, Ningyuan, NMHU.

Sichuana Shen \& Yin, 2020

Sichuana cryptospina Shen \& Yin, 2020

Yin et al. 2020. Zootaxa, 4786 (3): 369-380

Type specimens. Holotype: $\widehat{\partial}$ (nymph), China, Sichuan, Ngawa Tibetan and Qiang Autonomous Prefecture,

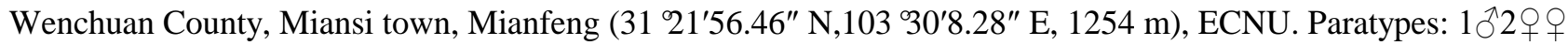
(nymphs), same data as in holotype; $1 \sigma^{\widehat{\lambda}}$ (nymphs), China, Sichuan, Ngawa Tibetan and Qiang Autonomous

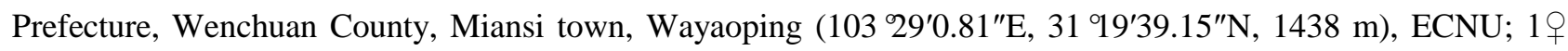
(nymphs), China, Sichuan, Ngawa Tibetan and Qiang Autonomous Prefecture, Wenchuan County, Weizhou town,

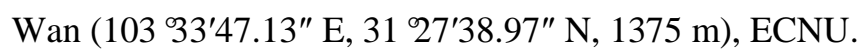

Sichuana feicui He, 2020

He et al. 2020. Zootaxa, 4877 (1): 195-200

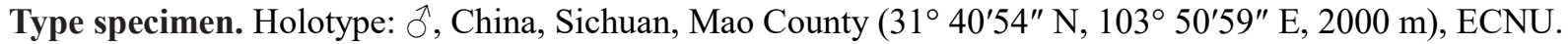

Sinocyrtaspis megalobia Wang, Xin \& Shi, 2020

Wang et al. 2020. Zootaxa, 4718 (4): 584-590

Type specimens. Holotype: $\widehat{\partial}$, China, Hunan, Yangmingshan, Shuangpai, NMHU. Paratypes: $1{ }^{\jmath} 1 \propto$, China, Hunan, Yangmingshan, Shuangpai, NMHU.

Paradoxitettigia Wang, Huang \& Shi, 2020

Paradoxitettigia longicaudica Wang, Huang \& Shi, 2020

Wang et al. 2020. Zootaxa, 4750 (1): 138-142

Type specimens. Holotype: $\hat{\alpha}$, China, Hunan, Hengshan, Hengyang, NMHU. Paratypes: $3 \hat{\delta} \hat{\jmath} 4 \hat{+} q$, same data as holotype, NMHU.

Pseudophlugiolopsis Wang, Zhou \& Chang, 2020

Pseudophlugiolopsis bitubera Wang, Zhou \& Chang, 2020

Wang et al. 2020. Zootaxa, 4802 (1): 182-188

Type specimens. Holotype: $\widehat{\jmath}$, China, Yunnan, Xinjie, Yuanyang, NMHU. Paratypes: $2 q \circ$, China, Yunnan, Xinjie, Yuanyang, NMHU.

Xiangothaumaspis Xin, Wang \& Shi, 2020 (湘杉蚉属)

Xiangothaumaspis falcatus Xin, Wang \& Shi, 2020 (镰尾湘杉蚉)

Xin et al. 2020. Zootaxa, 4819 (2): 385-392

Type specimens. Holotype: $\delta$, China, Hunan, Mangshan, Yizhang, NMHU. Paratypes: 1q, China, Hunan, Mangshan, Yizhang, NMHU.

Xiphidiopsis bifurcatis Cui, Liu \& Chang, 2020

Cui et al. Zootaxa, 4845 (1): 127-132

Type specimens. Holotype: $\hat{\sigma}$, China, Yunnan, Nabang, Yingjiang, NMHU. Paratypes: $1 \hat{0}$, China, Yunnan, Banhong, Cangyuan; 10ิ, China, Yunnan, Mengsong, Menghai, NMHU. 
刘童神, 陈静, 姜立云, 乔格侠 (2021) 中国半翅目等29目昆虫2020年新分类单元. 生物多样性, 29, 1050-1057. http://www.biodiversity-science.net/CN/10.17520/biods.2021200.

Xiphidiopsis spoona Cui, Liu \& Chang, 2020

Cui et al. Zootaxa, 4845 (1): 127-132

Type specimens. Holotype: $\hat{\delta}$, China, Yunnan, Daweishan, Pingbian, NMHU. Paratype: 1 , , other data same as holotype, NMHU.

Xizicus furcus Cui, Liu \& Shi, 2020

Cui et al. Zootaxa, 4845 (1): 127-132

Type specimens. Holotype: $\widehat{\partial}$, China, Yunnan, Gaoligongshan, Baoshan $\left(25.3023^{\circ} \mathrm{N}, 98.7952^{\circ} \mathrm{E}, 1800 \mathrm{~m}\right)$. Paratype: 1 , other data same to the holotype, NMHU.

Xizicus gaoligongshanensis Cui, Liu \& Shi, 2020

Cui et al. Zootaxa, 4845 (1): 127-132

Type specimens. Holotype: ${ }^{\lambda}$, China, Yunnan, Gaoligongshan, Baoshan $\left(25.2995^{\circ}\right.$ N, $98.7836^{\circ}$ E, 2060 m), NMHU. Paratype: 1 , other data same to the holotype, NMHU.

Zulpha ruohua Wu, Zhang \& Liu, 2020

Wu et al. 2020. Zootaxa, 4894 (1): 111-122

Type specimens. Holotype: $\widehat{\partial}$, Vietnam: Thanh Hóa (250-300m), IZCAS. Paratypes: 1 9 , China: Guangxi, GuilinMao'er Shan Mt. (500 m), IZCAS.

Zulpha fenghuang Wu \& Liu, 2020

Wu et al. 2020. Zootaxa, 4894 (1): 111-122

Type specimens. Holotype: $\partial^{\lambda}$, China, Yunnan, Dehong Dai-Jingpo Autonomous Prefecture, Ruili $\left(24.0708^{\circ} \mathrm{N}\right.$,

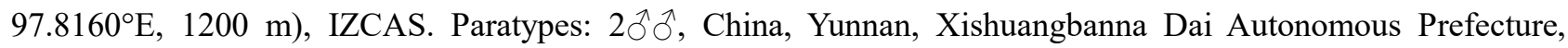
Mengla-Mohan $\left(21.2573^{\circ} \mathrm{N}, 101.7330^{\circ} \mathrm{E}, 980 \mathrm{~m}\right)$, IZCAS; $2 \hat{0}^{\hat{\alpha}}$, China, Yunnan, Xishuangbanna Dai Autonomous Prefecture, Jinghong-Jinuo (850 m); 10, PWC; 1ㅇ, China, Yunnan, Xishuangbanna Dai Autonomous Prefecture, Jinghong-Menglun $\left(21.9752^{\circ} \mathrm{N}, 101.1816^{\circ} \mathrm{E}, 910 \mathrm{~m}\right)$, IZCAS.

Tridactylidae

Xya xishangbanna Cao, Rong \& Naveed, 2020

Cao et al. 2020. ZooKeys, 947: 103-112

Type specimens. Holotype: $\widehat{\partial}$, China Yunnan, Xishuangbanna, Mengla County, Wuxiangguangchang (21.92 N, 101.11 E), LNU. Paratype: 1 q China, same data as holotype, LNU.

Xya yunnanensis Cao, Rong \& Naveed, 2020

Cao et al. 2020. ZooKeys, 947: 103-112

Type specimen. Holotype: $\widehat{\jmath}$, China, Yunnan, Xishuangbanna, Mengla County (22.05 N, 100.89 E), LNU.

Xya fujianensis Cao, Chen \& Yin, 2020

Cao et al. 2020. ZooKeys, 947: 103-112

Type specimens. Holotype: $\overbrace{}^{\lambda}$, China, Fujian, Sanming $\left(26^{\circ} 13^{\prime}\right.$ N, $117^{\circ} 36^{\prime}$ E), LNU. Paratypes: 2 $\uparrow$, China, Fujian, Sanming $\left(26^{\circ} 13^{\prime} \mathrm{N}, 117^{\circ} 36^{\prime} \mathrm{E}\right)$, LNU. 
刘童神, 陈静, 姜立云, 乔格侠 (2021) 中国半翅目等29目昆虫2020年新分类单元. 生物多样性, 29, 1050-1057. http://www.biodiversity-science.net/CN/10.17520/biods.2021200.

Trigonidiidae

Abstrigonidium He, 2020.

Emerasoma He, 2020

Emerasoma curvicerca He, 2020

He et al. 2020. Zootaxa, 4890 (1): 119-128

Type specimens. Holotype: §̊, China, Zhejiang, Lin’an, Tianmushan (30¹8'53" N, 119²7'23" E, 300 m), ECNU.

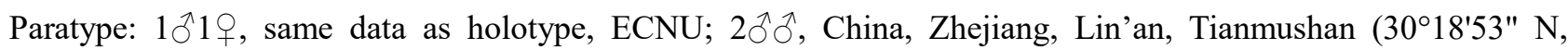

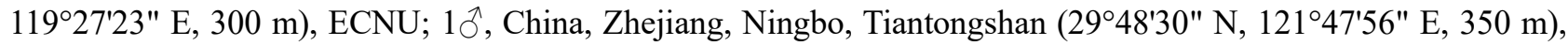
ECNU.

Giganemobius Shen \& He, 2020

Shen et al. 2020. Zootaxa, 4778 (2): 383-390

Homonemobius curifolis Zhang, Wang \& Liu, 2020

Wang et al. 2020. Zootaxa, 4729 (4): 575-581

Type specimens. Holotype: $\curvearrowright$, China, Yunnan, Lincang, Mt. wulao, ECNU. Paratype: 19 : same data the holotype, ECNU.

Pteronemobius truncates Zhang, Wang \& Liu, 2020

Wang et al. 2020. Zootaxa, 4729 (4): 575-581

Type specimens. Holotype: $\curvearrowright$, China, Yunnan, Cangyuan, Banhong, MHBU. Paratypes: $1 \overbrace{}^{\lambda} \propto q$, the data same as the holotype, MHBU; $2 \widehat{\jmath} 2$ 우, China, Yunnan, Longchuan, Long an, MHBU.

Qionemobius Shen \& He, 2020

Qionemobius hsterus Shen \& He, 2020

Shen et al. 2020. Zootaxa, 4778 (2): 383-390

Type specimens. Holotype: $\widehat{\jmath}$, China, Hainan, Ledong, Jianfengling Mt. (1843'14" N, 10852'12" E, 1380 m), ECNU. Paratypes: $2 \delta^{\top} 2 q 0$, same data as holotype, ECNU.

\section{蜼目 Phasmatodea}

Diapheromeridae

Acanthophasma brevicercum Ho, 2020

Ho. 2020. Hong Kong Entomological Bulletin, 12: 3-28

Type specimens. Holotype: $1 \lesssim$, China, Yunnan, Longchuan, Dehong (2000m), SEMCAS.

Acanthophasma dilatatum Ho, 2020

Ho. 2020. Hong Kong Entomological Bulletin, 12: 3-28

Type specimens. Holotype: $\stackrel{\jmath}{\sigma}$, China, Nanjian, Dali, Yunnan, 2000m, HKES. Paratype: 1 immature $q$, same data as holotype $\hat{\jmath}$, HKES.

\section{Cheniphasma parvidentatum Ho, 2020}

Ho. 2020. Hong Kong Entomological Bulletin, 12: 3-28

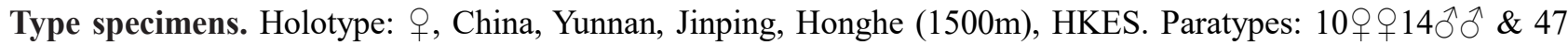
eggs (naturally laid by paratypes + ), same data as holotype ${ }_{+}$, HKES. 
刘童神, 陈静, 姜立云, 乔格侠 (2021) 中国半翅目等29目昆虫2020年新分类单元. 生物多样性, 29, 1050-1057. http://www.biodiversity-science.net/CN/10.17520/biods.2021200.

Oedohirasea Ho, 2020

Oedohirasea huanglianshanensis Ho, 2020

Ho. 2020. Hong Kong Entomological Bulletin, 12: 3-28

Type specimen. Holotype: $\widehat{\jmath}$, China, Yunnan, Huanglianshan, Luchun, Honghe, (1300-1400m), HKES.

Oxyartes densigranulatus Ho, 2020

Ho. 2020. Hong Kong Entomological Bulletin 12: 3-28

Type specimens. Holotype: $q$, China, Baoshan, Yunnan, 1500m, HKES. Paratypes: $4 \uparrow q 5 \hat{\jmath} \widehat{\delta} 19$ eggs (naturally laid by holotype $q \&$ paratypes $q$ ), same data as holotype $q$, HKES.

Oxyartes despectus yingjiangensis Ho, 2020

Ho. 2020. Hong Kong Entomological Bulletin 12: 3-28

Type specimens. Holotype: + , China, Yunnan, Yingjiang, Dehong $(1000 \mathrm{~m})$, HKES. Paratypes: $1 \uparrow 2 \hat{\jmath} \hat{\sigma}, 6$ eggs (naturally laid by holotype \& paratype + ), same data as holotype + , HKES.

Oxyartes nigrigranulatus $\mathrm{Ho}, 2020$

Ho. 2020. Hong Kong Entomological Bulletin 12: 3-28

Type specimens. Holotype: + , China, Yunnan, Xiping, Yuxi (2000 m), HKES. Paratypes: $2+q, 21$ eggs (naturally laid by paratypes $q$ ), same data as holotype $q$, HKES.

Oxyartes rubris distinctus $\mathrm{Ho}, 2020$

Ho. 2020. Hong Kong Entomological Bulletin 12: 3-28

Type specimens. Holotype: $q$, China, Jinping, Honghe, Yunnan (1500 m), HKES. Paratypes: $3+q+2 \hat{\jmath}, 27$ eggs (naturally laid by holotype \& paratypes + ), same data as holotype + , HKES.

Oxyartes sparsispinosus Ho, 2020

Ho. 2020. Hong Kong Entomological Bulletin 12: 3-28

Type specimens. Holotype: + , China, Baoshan, Yunnan, (2000 m), HKES. Paratypes: $4 \hat{\jmath} \hat{\jmath}$, same data as holotype +, HKES.

Oxyartes xishuangbannaensis Ho, 2020

Ho. 2020. Hong Kong Entomological Bulletin 12: 3-28

Type specimens. Holotype: $q$, China, Yunnan, Menglun, Xishuangbanna (500-600m), HKES. Paratypes: $2 q q$ $2 \widehat{\partial} \widehat{0}$, same data as holotype + , HKE.

Phasmatidae

Parapachymorpha apicalis George Ho, 2020

Ho. 2020. Hong Kong Entomological Bulletin, 12: 3-9

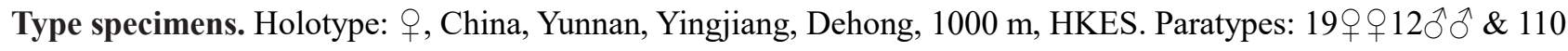
eggs (naturally laid by paratype + ), same data as holotype + , HKES.

Parapachymorpha tridentata George Ho, 2020

Ho. 2020. Hong Kong Entomological Bulletin 12: 3-9 
刘童神, 陈静, 姜立云, 乔格侠 (2021) 中国半翅目等29目昆虫2020年新分类单元. 生物多样性, 29, 1050-1057. http://www.biodiversity-science.net/CN/10.17520/biods.2021200.

Type specimens. Holotype: $q$, China, Yunnan, Bubang, Mengla, Xishuangbanna (500-600m), HKES. Paratypes: $4 ㅇ ㅜ 5 \hat{\jmath} \hat{\delta} \& 25$ eggs (naturally laid by paratype + ), same data as holotype + , HKES.

\section{凮目 Phthiraptera}

Leiothrichidae

Myrsidea attenuata Lei, Chu, Dik, Zou, Wang \& Gustafsson, 2020

Lei et al. 2020. Zootaxa, 4878 (1): 103-128

Type specimens. Holotype: $\hat{\partial}$, China, Guangdong, Nanling Reservation, Ruyang County, GIABR. Paratypes: $2 \circ$, GIABR. $3 \hat{\partial} \hat{\jmath}$, GIABR. 1 , , GIABR.

Myrsidea liopari Lei, Chu, Dik, Zou, Wang \& Gustafsson, 2020

Lei et al. 2020. Zootaxa, 4878 (1): 103-128

Type specimens. Holotype: $\delta$, China, Yunnan, Ailaoshan, Jingdong County (2420 m), GIABR. Paratypes: $2 \circ$,

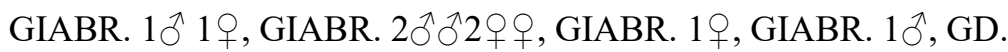

Myrsidea suthorae Lei, Chu, Dik, Zou, Wang \& Gustafsson, 2020

Lei et al. 2020. Zootaxa, 4878 (1): 103-128

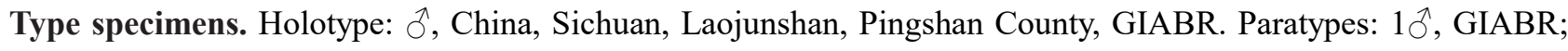
$1{ }^{\top}$, GIABR.

Myrsidea zhangae Lei, Chu, Dik, Zou, Wang \& Gustafsson, 2020

Lei et al. 2020. Zootaxa, 4878 (1): 103-128

Type specimens. Holotype: $\widehat{\jmath}$, China, Hunan, Badagongshan, Sangzhi County, GIABR. Paratypes: $8 \hat{\jmath} \widehat{\partial} 5$ 우 $ᄋ$, same data as holotype, GIABR.

Philopteridae

Guimaraesiella latitemporalis Gustafsson \& Bush, 2020

Gustafsson \& Bush. 2020. Zootaxa, 4885 (2): 151-188

Type specimens. Holotype: ${ }^{\lambda}$, Thailand, Ban Nong Wai, Na Phung, Dan Sai District, Loei, NHML. Paratypes:

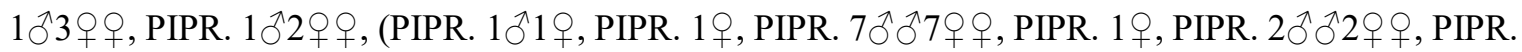

Cataphractomimus Gustafsson, Lei, Chu \& Zou, 2020

Cataphractomimus impervius Gustafsson, Lei, Chu \& Zou, 2020

Gustafsson et al. 2020. Zootaxa, 4742 (2): 201-255

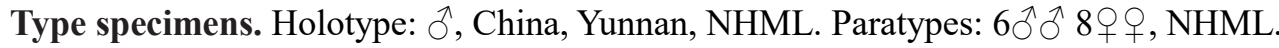

Cataphractomimus mirapelta Gustafsson, Lei, Chu \& Zou, 2020

Gustafsson et al. 2020. Zootaxa, 4742 (2): 201-255

Type specimens. Holotype: $\curvearrowright$, China, Sichuan, "Upper Minho" [= Upper Min River, China], NHML. Paratype: $1{ }^{\lambda}$, NHML.

Cataphractomimus junae Gustafsson, Lei, Chu \& Zou, 2020

Gustafsson et al. 2020. Zootaxa, 4742 (2): 201-255

Type specimens. Holotype: $\hat{\partial}$, China, Sichuan, NHML. Paratypes: $2 \hat{\jmath} \widehat{\jmath}$, NHML. 
刘童神, 陈静, 姜立云, 乔格侠 (2021) 中国半翅目等29目昆虫2020年新分类单元. 生物多样性, 29, 1050-1057. http://www.biodiversity-science.net/CN/10.17520/biods.2021200.

Megalipeurus sinensis Gustafsson, Lei, Chu \& Zou, 2020

Gustafsson et al. 2020. Zootaxa, 4742 (2): 201-255

Type specimens. Holotype: $\widehat{\partial}$, China, NHML. Paratype: $1 \delta$, NHML.

Sinolipeurus Gustafsson, Lei, Chu \& Zou, 2020

Sinolipeurus sichuanensis Gustafsson, Lei, Chu \& Zou, 2020

Gustafsson et al. 2020. Zootaxa, 4742 (2): 201-255

Type specimen. Holotype: $\widehat{\partial}$, China, Sichuan, NHML.

\section{禎翅目 Plecoptera}

Capniidae

Eucapnopsis yunnana Cao, Yang \& Li, 2020

Cao et al. 2020. Zootaxa, 4779 (1): 142-148

Type specimens. Holotype: $\partial^{\Uparrow}$, China, Yunnan, Deqing County, Baima Mountain ( $\left.98^{\circ} 57^{\prime} \mathrm{E}, 27^{\circ} 24^{\prime} \mathrm{N}, 4295 \mathrm{~m}\right)$, HIST. Paratypes: $5 \hat{\delta} 4$ 웅, same locality and data as holotype, 2 우 $ᄋ$, same locality and collector, HIST.

Sweltsa bilobata Mo, Ye, Wang \& Li, 2020

Chloroperlidae

Mo et al. 2020. Zootaxa, 4853 (2): 275-282

Type specimens. Holotype: $\widehat{\jmath}$, China, Guangxi Zhuang Autonomous Region, Guilin City, Xing'an County, Maoershan National Natural Reserve, Jiuniutang, (E 110²5'1’, N 2552’16’, 1961-2000 m), HIST. Paratypes: $1 \lesssim 1 \%$, same data and date as holotype, HIST.

Cryptoperla zhejiangensis Chen, 2020

Peltoperlidae

Chen. 2020. Zootaxa, 4810 (3): 576-588

Type specimens. Holotype: $\overbrace{}^{\AA}$, China, Zhejiang, Quzhou City, Housuntang $\left(28.81113727^{\circ} \mathrm{N}, 118.97745093^{\circ} \mathrm{E}, 640\right.$ m), ICJUST. Paratypes: $1 \delta^{\lambda}$, same locality and date as holotype; 19 , Zhejiang, Quzhou City, Mt. Yaowangshan, upstream of Housuntang (28 $\left.52^{\prime} 42.63^{\prime \prime} \mathrm{N}, 118^{\circ} 55^{\prime} 22.45^{\prime \prime} \mathrm{E}, 250 \mathrm{~m}\right)$, ICJUST.

\section{Perlidae}

Caroperla brevihamata Mo, Wang \& Li, 2020

Mo et al. 2020. Zootaxa, 4869 (3): 430-436

Type specimens. Holotype: $\widehat{O}$, China, Guangxi Zhuang Autonomous Region, Guilin City, Multinational Autonomous County of Longsheng, Huaping National Nature Reserve (253’ 37’ N, 10954’42” E, 731.6m), HIST . Paratype: 1 , same location and collectors as holotype, HIST.

Etrocorema yunnanensis Chen, 2020

Chen. 2020. Zootaxa, 4759 (3): 433-439

Type specimens. Holotype: $\sigma^{\prime}$, China, Yunnan, Mengla County, Laman Highway $\left(21^{\circ} 20^{\prime} 53.25^{\prime \prime} \mathrm{N}, 101^{\circ} 18^{\prime} 33.79^{\prime \prime} \mathrm{E}\right.$, $1400 \mathrm{~m})$, ICJUST. Paratypes: $1 \overbrace{}^{\Uparrow} 1$ 울, same data as holotype, ICJUST. 
刘童神, 陈静, 姜立云, 乔格侠 (2021) 中国半翅目等29目昆虫2020年新分类单元. 生物多样性, 29, 1050-1057. http://www.biodiversity-science.net/CN/10.17520/biods.2021200.

Mo et al. 2020. Zootaxa, 4732 (4): 565-572

Type specimens. Holotype: $\widehat{\partial}$, China, Guangxi Zhuang Autonomous Region, Guilin City, Multinational Autonomous County of Longsheng, at gate of Huaping National Nature Reserve (2537'37' N, 109 54'41' E, 734 m), HIST. Paratypes: $4 \hat{\jmath} \hat{\delta} 1$, HIST, same data and date as holotype; 2 , , HIST.

Hemacroneuria baotianmana Chen \& Hou, 2020

Chen \& Hou. 2020. Zootaxa, 4751 (1): 161-171

Type specimens. Holotype: $\hat{\sigma}$, China, Henan, Nanyang City, Neixiang County, Baotianman Scenic Area

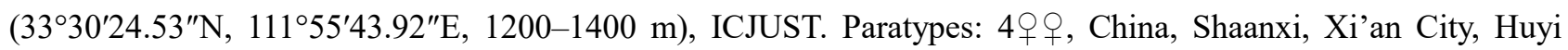

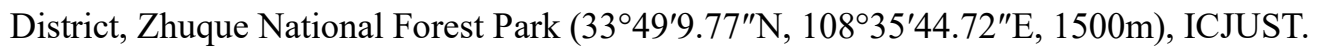

Hemacroneuria trilobata Mo, Wang \& Li, 2020

Mo et al. 2020. Zootaxa, 4780 (2): 396-400

Type specimen. Holotype: $\partial^{\lambda}$, Guangxi Zhuang Autonomous Region, Tianlin County, Cenwanglaoshan ( $24^{\circ} 29.25^{\prime}$ $\left.\mathrm{N}, 106^{\circ} 23.48^{\prime} \mathrm{E}, 1849 \mathrm{~m}\right)$, HIST.

Kamimuria shiae Li, Kong \& Liu, 2020

Li et al. 2020. Zootaxa, 4809 (2): 363-373

Type specimens. Holotype: $\widehat{\partial}$, China, Fujian, Nanping City, Wuyishan, Guadun, (N 2744'12", E 117³8'12", 1200 m), HIST. Paratype: 1 ${ }^{\lambda}$, China, Fujian, Shaowu County, Wuyishan, Nanhu Forest Farm (N 27³2’38”, E 117²9’3”, 409m), HIST.

Kamimuria tenuilobata Li, Kong \& Liu, 2020

Li et al. 2020. Zootaxa, 4809 (2): 363-373

Type specimens. Holotype: $\widehat{O}$, China, Fujian, Nanping City, Shaowu County, Wuyishan, Nanhu Forest Farm, ( N 27³2’38”, E 117²9’3”, 409m), HIST. Paratype: 10^, same locality, HIST.

Kiotina yexiaohani Su \& Chen, 2020

Su \& Chen. 2020. Zootaxa, 4786 (4): 583-591

Type specimens. Holotype: $\overbrace{}^{\top}$, China, Zhejiang, Quzhou City, Shuimenjian $\left(28.76842255^{\circ} \mathrm{N}, 118.93807864^{\circ} \mathrm{E}, 620\right.$ m), ICJUST. Paratype: 1 , same locality and date as holotype, ICJUST.

Neoperla delphina Li, Mo \& Wang, 2020

Li et al. 2020. Zootaxa, 4718 (4): 573-583

Type specimens. Holotype: $\widehat{A}$, China, Guangxi, Laibing City, Jinxiu County, Dayao Mountain, Yinshan Station.

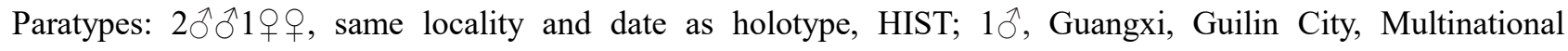

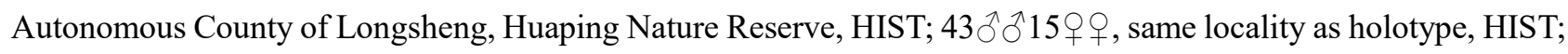
$50{ }^{\lambda} 8$ 우, Guangxi, Laibing City, Jinxiu County, Dayao Mountain, Yinshan Park, 1150m, HIST; $1{ }^{\lambda} 2+q$, Guangxi, Guilin City, Multinational Autonomous County of Longsheng, ( N 2553.6', E 110¹2.4', 360m), NMPCR;

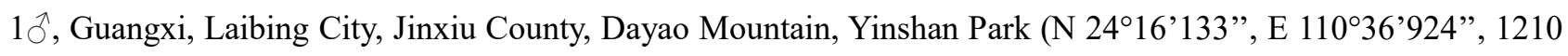
m), HIST.

Neoperla quadrispina Li, Mo \& Wang, 2020

Li et al. 2020. Zootaxa, 4718 (4): 573-583 
刘童神, 陈静, 姜立云, 乔格侠 (2021) 中国半翅目等29目昆虫2020年新分类单元. 生物多样性, 29, 1050-1057. http://www.biodiversity-science.net/CN/10.17520/biods.2021200.

Type specimens. Holotype: $\hat{\jmath}$, China, Guangxi, Guigang City, Tianping Mountain National Forest Park, 210m, HIST. Paratype: $1 \lesssim$, same locality and date as holotype, HIST.

Neoperla curvilobata Mo, Wang \& Li, 2020

Mo et al. 2020. Zootaxa, 4790 (3): 515-524

Type specimen. Holotype: $\hat{\jmath}$, China, Guangxi Zhuang Autonomous Region, Nanning City, Wuming County, Liangjiang Town, Neichao, Neichao River above Neichao Ming Hotel (2329.664'N, 108²1.622'E, 220 m), HIST.

Neoperlops triangulates Mo, Wang, Li \& Murányi, 2020

Mo et al. 2020. Zootaxa, 4763 (3): 405-418

Type specimens. Holotype: $\delta$, China, Guangxi Zhuang Autonomous Region, Shangsi County, Shiwanda Mts, Wanggui Village, Pinglong River at the village $\left(21^{\circ} 52.411^{\prime} \mathrm{N}, 107^{\circ} 49.996^{\prime} \mathrm{E}, 260 \mathrm{~m}\right)$. Paratypes: $3 \overbrace{}^{\lambda} 1{ }^{\circ}$ same data as holotype, HIST; $2 \hat{\delta} 1 q$ with its exuviae, $3 \hat{\jmath} \widehat{\sigma}$ and 3 우 exuviae HNHM; Guangxi, Shangsi County, Shiwandashan National Forest Park, Minan River (2151.869' N, 10750.712' E, 300m), HIST; 19 larva, HNHM; Guangxi, Shangsi County, Shiwandashan National Forest Park, Pearl River below tourist route bridge $\left(21^{\circ} 54.122^{\prime}\right.$ N, 10754.292' E, 315m), HNHM.

Phanoperla huanghuye Chen, 2020

Chen. 2020. Zootaxa, 4718 (2): 230-238

Type specimens. Holotype: $\widehat{\jmath}$, China, Fujian, Wuyishan City, Xingcun Town, Tongmu Village, near Mt. Wuyishan, unnamed stream $\left(27.7527\right.$ N, 117.6882 E, 800m), ICJUST. Paratypes: $4 \delta^{\lambda} 6 q q$, same data as holotype, ICJUST.

Sinacroneuria huzhengkuni Du \& Huo, 2020

Hou \& Du. 2020. Zootaxa, 4834 (2): 283-292

Type specimen. Holotype: $\hat{\sigma}$, China, Guizhou, Tongren City, Mount Fanjing (Fanjingshan), Yamugou stream $\left(108.73^{\circ} \mathrm{E}, 27.80^{\circ} \mathrm{N}\right)$, ICYZU.

Sinacroneuria lii Huo \& Du, 2020

Hou \& Du. 2020. Zootaxa, 4834 (2): 283-292

Type specimen. Holotype: $\widehat{\jmath}$, China, Guizhou, Zunyi City, Suiyang County, Kuankuoshui National Nature Reserve, beside a nameless road $\left(28^{\circ} 13.205^{\prime} \mathrm{N}, 107^{\circ} 9.95^{\prime} \mathrm{E}, 1435 \mathrm{~m}\right), \mathrm{ICYZU}$.

Sinacroneuria fujianensis Sivec \& Stark, 2020

Sivec \& Stark. 2020. Zootaxa, 4732 (4): 580-584

Type specimen. Holotype: $\widehat{\partial}$, China, Fujian, Kuatun [Wuyishan City, Wuyishan Mountain, Guadun], ZFMK.

Sinacroneuria lateralis Sivec \& Stark, 2020

Sivec \& Stark. 2020. Zootaxa, 4732 (4): 580-584

Type specimen. Holotype: $\widehat{\jmath}$, China, Fujian, Kuatun, 2300 m, ZFMK.

Tyloperla bimaculata Li \& Kong, 2020

Li \& Kong. 2020. Zootaxa, 4728 (2): 289-294

Type specimen. Holotype: $\widehat{\jmath}$, China, Guizhou, Suiyang County, Kuankuoshui National Nature Reserve $\left(107^{\circ} 19.98\right.$ E, $\left.28^{\circ} 24.03 \mathrm{~N}, 300 \mathrm{~m}\right), \mathrm{CAU}$. 
刘童神, 陈静, 姜立云, 乔格侠 (2021) 中国半翅目等29目昆虫2020年新分类单元. 生物多样性, 29, 1050-1057. http://www.biodiversity-science.net/CN/10.17520/biods.2021200.

Tyloperla bilobata Mo, Wang \& Li, 2020

Mo et al. 2020. Zootaxa, 4763 (1): 109-116

Type specimen. Holotype: $\circ$, China, Guangxi, Fangcheng, Jinhuacha Nature Reserve ( $\left.21^{\circ} 76,09^{\prime} \mathrm{N}, 108^{\circ} 43,49^{\prime} \mathrm{E}\right)$, CAU.

Perlodidae

Filchneria dongruihangi Chen, 2020

Chen. 2020. Zootaxa, 4808 (2): 350-364

Type specimens. Holotype: $\widehat{\jmath}$, China, Heilongjiang, Qiqihar City, Liuyuan Park, Nenjiang River (47.35992625 N, $123.90812588 \mathrm{E}, 145 \mathrm{~m})$, ICJUST. Paratypes: $2 \hat{\delta} \widehat{\delta} 1$ 우우, same locality and data as holotype, ICJUST.

Isoperla sejila Cao, Wang \& $\mathrm{Li}, 2020$

Cao et al. 2020. Zootaxa, 4779 (1): 142-148

Type specimens. Holotype: $\widehat{\partial}$, China, Tibet, Nyingchi City, Nyingchi County, Sejilashan (Sejila Mountains),

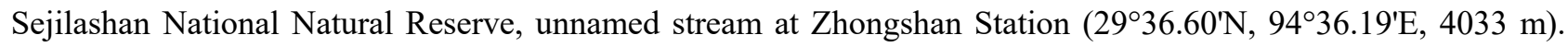

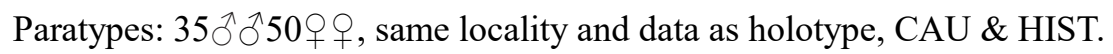

Isoperla sextuberculata Huo \& Du, 2020

Huo \& Du. 2020. Zootaxa, 4801 (3): 552-558

Type specimens. Holotype: ${ }^{\top}$, China, Hubei, Shennongiia area, Guitouwan $\left(110^{\circ} 08.872^{\prime} \mathrm{E}, 31^{\circ} 28.439^{\prime} \mathrm{N}, 2150 \mathrm{~m}\right)$, ICYZU. Paratypes: $2 q 9$, the same data and locality as the holotype, ICYZU.

Sinoperlodes Chen, 2020

Sinoperlodes zhouchangfai Chen, 2020

Chen. 2020. Zootaxa, 4779 (4): 584-594

Type specimens. Holotype: $\widehat{\partial}$, China, Zhejiang, Hangzhou City, Tianmu Mountain, Tianmu Stream (30.3604 N, 119.4434 E, 580 m), ICJUST. Paratypes: 4우, same locality and data as holotype, ICJUST.

Parisoperla Huo \& Du, 2020

Parisoperla kuankuoshuiensis Du \& Huo, 2020

Hou \& Du. 2020. Zootaxa, 4718 (4): 470-480

Type specimens. Holotype: $\widehat{\partial}$, China, Guizhou, Kuankuoshui National Nature Reserve, beside a nameless road $\left(28^{\circ} 13.205^{\prime} \mathrm{N}, 107^{\circ} 9.95^{\prime} \mathrm{E}, 1435 \mathrm{~m}\right)$, ICYZU. Paratypes: $10^{\Uparrow} 1$ \% , the same locality and data as holotype, ICYZU.

Parisoperla leigongshana Huo \& Du, 2020

Hou \& Du. 2020. Zootaxa, 4718 (4): 470-480

Type specimen. Holotype: ${ }^{\lambda}$, China, Guizhou, Leigongshan Nature Reserve, beside an unnamed road (26.3616N, 108.1650E, 1198m), ICYZU.

Amphinemura jiaoheensis Chen, 2020

Nemouridae

Chen. 2020. European Journal of Taxonomy, 651: 1-16

Type specimens. Holotype: $\delta^{\lambda}$, China, Jilin, Jiaohe City, Sidaogou Mountain, a small unnamed stream $\left(43^{\circ} 44^{\prime} 40^{\prime \prime}\right.$ 
刘童神, 陈静, 姜立云, 乔格侠 (2021) 中国半翅目等29目昆虫2020年新分类单元. 生物多样性, 29, 1050-1057. http://www.biodiversity-science.net/CN/10.17520/biods.2021200.

N, $\left.127^{\circ} 03^{\prime} 05^{\prime \prime} \mathrm{E} ; 600 \mathrm{~m}\right)$, ICJUST. Paratypes: $1{ }^{\Uparrow} 2+$ 우, same collection data as for holotype, ICJUST.

Amphinemura bifascia Mo, Wang, Yang \& Li, 2020

Mo et al. 2020. Zootaxa, 4751 (1): 131-142

Type specimens. Holotype: $\hat{\partial}$, China, Guangxi, Nanning City, Wuming County, Damingshan National Natural Reserve (N 2330', E 108²6', 1230 m), CAU. Paratypes: 1ㅇ, same locality and date as holotype, HIST; $29+$,

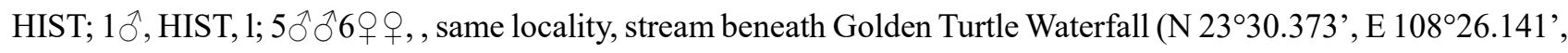

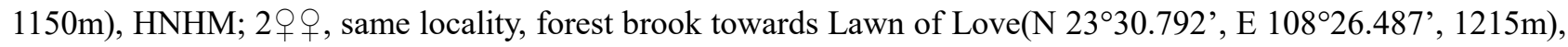

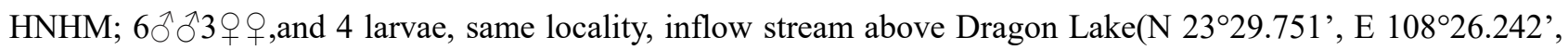
$1225 \mathrm{~m})$, HNHM.

Amphinemura bicornata Mo, Wang, Yang \& Li, 2020

Mo et al. 2020. Zootaxa, 4751 (1): 131-142

Type specimen. Holotype: $\widehat{\jmath}$, China, Guangxi, Nanning City, Wuming County, Damingshan National Natural Reserve (N 233', E 108²6’, 300 m), HIST.

Amphinemura changbaishana Li, Teslenko \& Yang, 2020

Li et al. 2020. Zootaxa, 4743 (3): 402-410

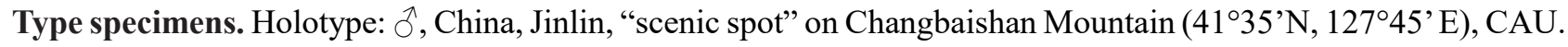
Paratype: $1 \hat{\delta}$, same locality and date as holotype, HIST.

Amphinemura qiliana Li, Teslenko \& Yang, 2020

Li et al. 2020. Zootaxa, 4743 (3): 402-410

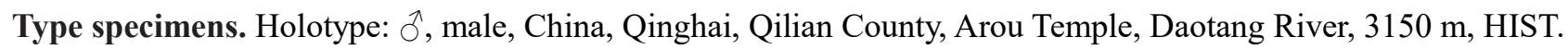
Paratype: $1{ }^{\lambda}$, same locality and date as holotype, HIST

Amphinemura retusilobata Mo, Wang, Yang, Li \& Murányi, 2020

Mo et al. 2020. Zootaxa, 4820 (2): 337-350

Type specimens. Holotype: $\widehat{\jmath}$, China, Guangdong, Maoming City, Xinyi City, Dacheng Town, Yunkaishan National Natural Reserve, the bridge next to the security office at the entrance to the reserve( $22^{\circ} 16^{\prime} 34^{\prime \prime} \mathrm{N}, 111^{\circ} 11^{\prime} 43^{\prime \prime} \mathrm{E}, 966$

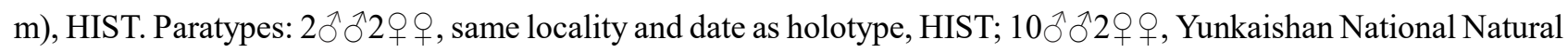

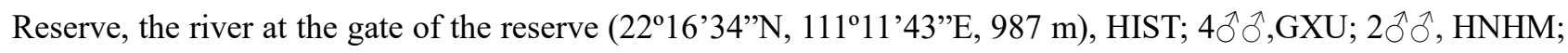

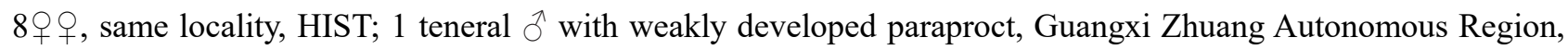

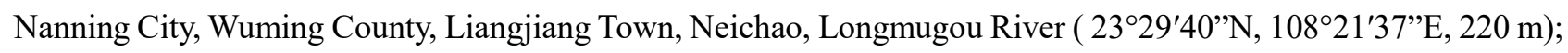

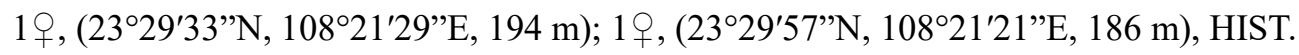

Amphinemura simifleurdelia Mo, Wang, Yang, Li \& Murányi, 2020

Mo et al. 2020. Zootaxa, 4820 (2): 337-350

Type specimens. Holotype: $\sigma^{\uparrow}$, China, Guangdong, Maoming City, Xinyi City, Dacheng Town, Yunkaishan National Natural Reserve, the bridge next to the security office at the entrance to the reserve $\left(22^{\circ} 16^{\prime} 34^{\prime \prime} \mathrm{N}, 111^{\circ} 11^{\prime} 43^{\prime \prime} \mathrm{E}\right.$,

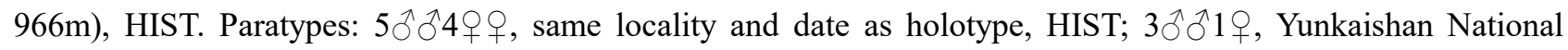
Natural Reserve, the river at the gate of the reserve $\left(22^{\circ} 16^{\prime} 34^{\prime \prime} \mathrm{N}, 111^{\circ} 11^{\prime} 43^{\prime \prime} \mathrm{E}, 987 \mathrm{~m}\right)$, HIST; $50^{\wedge} 0^{\wedge} 5$ 우, GXU;

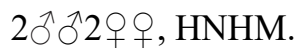


刘童神, 陈静, 姜立云, 乔格侠 (2021) 中国半翅目等29目昆虫2020年新分类单元. 生物多样性, 29, 1050-1057. http://www.biodiversity-science.net/CN/10.17520/biods.2021200.

Indonemoura maoershana Mo, Wang, Yang \& Li, 2020

Mo et al. 2020. Zootaxa, 4801 (2): 395-400

Type specimen. Holotype: đ̊, China, Guangxi Zhuang Autonomous Region, Guilin City, Xing'an County, Tongrencun, Maoershan National Natural Reserve, Jiulongtang, CAU.

Indonemoura wangae $\mathrm{Li}$, Yang \& Li, 2020

Li et al. 2020. Zootaxa, 4868 (3): 441-447

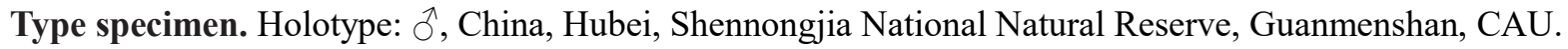

Nemoura cucurbitata Mo, Wang \& Li, 2020

Mo et al. 2020. Zootaxa, 4731 (1): 145-150

Type specimens. Holotype: $1 \widehat{\jmath}$, China, Guangxi Zhuang Autonomous Region, Guilin City, Xing'an County, Tongrencun, Maoershan National Natural Reserve $\left(25.905^{\circ} \mathrm{N}, 110.440^{\circ} \mathrm{E}, 1873.0 \mathrm{~m}\right)$, HIST.

Nemoura lixiana Chen, 2020

Chen. 2020. European Journal of Taxonomy, 651: 1-16

Type specimens. Holotype: $\widehat{\sigma}$, china, Sichuan, Lixian County, side of Xuemeng Road, a small unnamed stream $\left(31^{\circ} 33^{\prime} 56^{\prime \prime} \mathrm{N}, 103^{\circ} 17^{\prime} 32^{\prime \prime} \mathrm{E} ; 1634 \mathrm{~m}\right)$, ICJUST. Paratypes: $8 \overbrace{}^{\Uparrow} 4 ㅇ$ ${ }^{\circ}$, same collection data as for holotype, ICJUST.

Sinonemura Mo, Li \& Muranyi, 2020

Sinonemura balangshana Mo, Li \& Muranyi, 2020

Mo et al. 2020. PLoS One, 0229120

Type specimens. Holotype: $\widehat{\jmath}$, china, Sichuan, Tibetan Qiang Autonomous Prefecture of Ngawa, Wenchuan County, Balangshan(N 30.89905, E 102.91166, 3993 m), CAU. Paratypes: 2 9 , HIST; 1 , same data as holotype, HNHM.

Sphaeronemoura acutispina Mo, Wang, Yang \& Li, 2020

Mo et al. 2020. Zootaxa, 4763 (3): 447-450

Type specimen. Holotype: $\widehat{\jmath}$, China, Guangxi, Tianlin County, Cenwanglaoshan National Nature Reserve, Dalongping Protection Management Station (N 24²1', E 10615', 1300 m), CAU.

Sphaeronemoura multicurvata Li, Li \& Yang, 2020

Li et al. 2020. Zootaxa, 4722 (3): 234-240

Type specimens. Holotype: $\widehat{\jmath}$, China, Sichuan, Pingwu County, Old creek (not a stream name) (N 32³0’, E 104³8', 1400-1800m), CAU. Paratype: 1q, same data as holotype, CAU.

Sphaeronemoura tetragona Du \& Zhao, 2020

Zhao \& Du. 2020. Zootaxa, 4851 (3): 583-592

Type specimens. Holotype: $\curvearrowright$, China, Hunan, Taoyuan County, Yunwujie Nature Reserve, ICYZU. Paratypes: $1 \precsim$, the same data as holotype, ICYZU.

Protonemura longispina Zhao \& Du, 2020

Zhao \& Du. 2020. Zootaxa, 4802 (2): 250-260

Type specimens. Holotype: ${ }^{\top}$, China, Sichuan, Luding County, Yangjiageng River (N 2952.877'E $102^{\circ} 01.193^{\prime}$, 3646m), ICYZU. Paratypes: 390 , the same data as the holotype, ICYZU. 
刘童神, 陈静, 姜立云, 乔格侠 (2021) 中国半翅目等29目昆虫2020年新分类单元. 生物多样性, 29, 1050-1057. http://www.biodiversity-science.net/CN/10.17520/biods.2021200.

\section{原尾目 Protura}

Berberentulidae

Baculentulus wenyingae $\mathrm{Bu}, \mathrm{Gao} \&$ Luan, 2020

Bu et al. 2020. Entomotaxonomia, 42(3): 163-177

Type specimens. Holotype: + , china, Zhejiang, Zhoushan City, Dinghai District, Mahuangshan hill $\left(30^{\circ} 03^{\prime} \mathrm{N}\right.$, $122^{\circ} 03^{\prime} \mathrm{E}, 177 \mathrm{~m}$,), HUANG. Paratype: 1 , , same data as holotype, SNHM.

\section{Eosentomidae}

Pseudanisentomon lishuiensis $\mathrm{Bu}, \mathrm{Gao} \&$ Luan, 2020

Bu et al. 2020. Entomotaxonomia, 42(3): 163-177

Type specimens. Holotype: $\widehat{O}$, China, Zhejiang, Lishui City, Jingning County, Jiulongshan Geological Park $\left(41^{\circ} 30^{\prime} \mathrm{N}, 119^{\circ} 21^{\prime} \mathrm{E}\right.$, elev. $\left.1200 \mathrm{~m}\right)$, HUANG; Paratypes: $10^{\Uparrow}$, same data as holotype; 2 q $ᄋ$, Zhejiang, Lishui City,

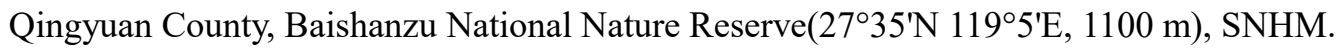

\section{蚤目 Siphonaptera}

Ceratophyllidae

Rowleyella fugongensis Gong \& Li, 2020 福贡罗氏蚤

Gong \& Li. 2020. Jishengchong Yu Yixue Kunchong Xuebao, 27 (1): 52-56

Type specimens. Holotype: $\widehat{O}^{\top}$, China, northwest Yunnan, Fugong County ( $\left.27^{\circ} 00^{\prime} \mathrm{N}, 98^{\circ} 50^{\prime} \mathrm{E}\right)$, AMMC. Paratype: 1 ,, YEDCP

\section{缨翅目 Thysanoptera}

Aeolothripidae

Streothrips jianshuiensis Liu, Mound \& Zhang, 2020

Liu et al. 2020. Zootaxa, 4819 (1): 001-005

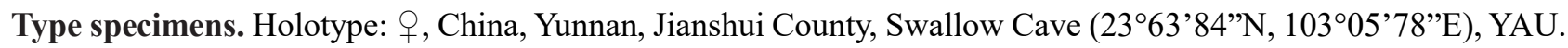
Paratypes: 1 , , same data as holotype; 1 , same locality, YAU.

\section{Phlaeothripidae}

Allothrips yunnanensis Mound, Li \& Zhang, 2020

Mound et al. 2020. Zootaxa, 4732 (4): 593-595

Type specimens. Holotype: female aptera, China, Yunnan, Kunming City, Arboretum (N 25 $08^{\prime}$ E $102^{\circ} 43^{\prime}$, 2006m), YAU. Paratypes: Paratypes, 49 female aptera, 6 female macropterae, China, Yunnan, Kunming City, Arboretum (N 25 $08^{\prime}$ E $\left.102^{\circ} 43^{\prime}, 2006 \mathrm{~m}\right)$, YAU, ANIC

Hoplothrips hongruiae Mound \& Wang, 2020

Mound \& Wang. 2020. Zootaxa, 4758 (3): 596-599

Type specimens. Holotype: macropterae female, China, Yunnan, Kunming, Western Mts, YAU. Paratypes: 8 macropterous females, 2 micropterous females, 7 macropterous males, 4 micropterous males, ANIC, JLU.

Liothrips dayulingensis Wang \& Lin, 2020

Wang \& Lin. 2020. Zootaxa, 4861 (3): 349-375

Type specimens. Holotype: + , China, Taiwan, Dayuling (Nantou), TARI. Paratypes: $1 \uparrow 1 \AA$, same data as holotype, TARI. 
刘童神, 陈静, 姜立云, 乔格侠 (2021) 中国半翅目等29目昆虫2020年新分类单元. 生物多样性, 29, 1050-1057. http://www.biodiversity-science.net/CN/10.17520/biods.2021200.

Liothrips hsuae Wang \& Lin, 2020

Wang \& Lin. 2020. Zootaxa, 4861 (3): 349-375

Type specimens. Holotype: $q$, China, Taiwan, Nanjan Mt. (Kendin), TARI. Paratype: $1+$ with same data as holotype, TARI.

Mesandrothrips acutisetis Dang, Zhao, Xie, Zhao \& Qiao, 2020

Dang et al. 2020. Zootaxa, 4816 (1): 123-128

Type specimens. Holotype: + , China, Shaanxi, Changqing National Nature Reserve, Yangjia ravine, SUT. Paratypes: $2 q q 1 \hat{\jmath}$, same data as holotype, SUT \& IZCAS. $1 q 1 \hat{\jmath}$, Sichuan, Guangyuan City, Cangwangxia Reserve, SUT.

Psephenothrips baiheensis Wang \& Lin, 2020

Wang \& Lin. 2020. Zootaxa, 4861 (3): 349-375

Type specimens. Holotype: + , China, Taiwan, Guanziling (Baihe), TARI. Paratypes: $1 \uparrow 1 \hat{\jmath}$, same data as holotype, TARI.

Psephenothrips cymbidas Wang \& Lin, 2020

Wang \& Lin. 2020. Zootaxa, 4861 (3): 349-375

Type specimens. Holotype: + , China, Taiwan, Puli (Nantou), TARI. Paratypes: $5+q 3 \hat{\jmath} \widehat{\jmath}$, same data as holotype, TARI.

\section{Thripidae}

Ctenothrips parisae Wang, Li, Tong \& Mound, 2020

Wang et al. 2020. Zootaxa, 4750 (3): 301-327

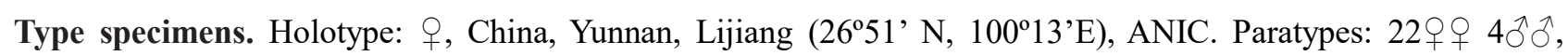
collected with holotype, YAU, ANIC.

Filicopsothrips pulcher Li, Yuan \& Zhang, 2020

Li et al. 2020. Zootaxa, 4845 (2): 297-300

Type specimens. Holotype: $q$, China, Yunnan, Maguan County, YAU. Paratypes: 19 , collected with holotype; $2 q q$, Yunnan, Xichou County, ANIC; 1 , Yunnan, Xishuangbanna Tropic Botanic Garden; ANIC.

Parabaliothrips robustus Masumoto \& Okajima, 2020

Masumoto \& Okajima. 2020. Zootaxa, 4838 (4): 566-580

Type specimens. Holotype: + , China, Taiwan, Nantou-hsien, Tsuifeng, 2300m, TUA. Paratypes: 146 $q+72 \hat{\jmath}$, same data as the holotype, TUA.

\section{毛翅目 Trichoptera}

Leptoceridae

Ceraclea (Athripsodina) aerumnula Yang \& Morse, 2020

Yang et al. 2020. Zootaxa, 4732 (1): 138-160

Type specimens. Holotype: $\stackrel{\lambda}{ }$, China, Zhejiang, City of Tai Zhou, Lin-hai County, Kuo-cang-shan [Mt.] (N28.83,

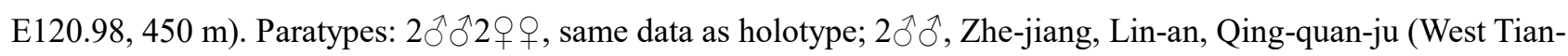


刘童神, 陈静, 姜立云, 乔格侠 (2021) 中国半翅目等29目昆虫2020年新分类单元. 生物多样性, 29, 1050-1057. http://www.biodiversity-science.net/CN/10.17520/biods.2021200.

mu Mt.), Chi-wu-li (N30.50, E119.60, 263 m); 10ภð. Zhe-jiang, An-ji, Long-wang-shan, $400 \mathrm{~m}$.

Ceraclea (Athripsodina) lamellata Yang \& Hu, 2020

Yang et al. 2020. Zootaxa, 4732 (1): 138-160

Type specimens. Holotype: ${ }^{\lambda}$, China, An-hui, Qi-men County, Lu-xi Town, Dao-hu Village (N29.52, E117.42, 200 m). Paratypes: $49 \precsim \widehat{\jmath} 255$ 우, same data as holotype.

Ceraclea (Ceraclea) megalophyllon Yang \& Morse, 2020

Yang et al. 2020. Zootaxa, 4732 (1): 138-160

Type specimens. Holotype: ${ }^{\lambda}$, China, Guang-xi, Xi-ning City, Wu-ming District, Da-Ming-shan [Mt.](N23.4964, E108.4401, $1254 \mathrm{~m})$. Paratype: 1 \% , same data as holotype.

Oecetis (Oecetis) discedens Yang \& Morse, 2020

Yang et al. 2020. Zootaxa, 4732 (1): 138-160

Type specimens. Holotype: ${ }^{\top}$, China Guang-xi: Xi-ning City, Wu-ming District, Da-Ming-shan [Mt.] (N23.496458,

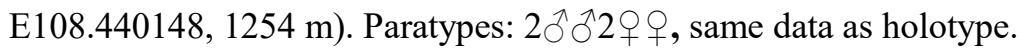

Oecetis (Pleurograpta) spinellosa Yang \& Hu, 2020

Yang et al. 2020. Zootaxa, 4732 (1): 138-160

Type specimens. Holotype: ${ }^{\curvearrowright}$, China, An-hui, Qi-men County, Li-xi Village, Qi-yang-keng(N29.52, E117.42, alt. 360 m). Paratypes: Same data as holotype, Gu-niu-jiang Nature Preserve Shuang-he-kou, Tao-yuan-li stream, 480 m, $2 \hat{\jmath} \partial^{\Uparrow} 11$ 우 $ᄋ$; Same data as holotype, except Peng-long, Xiang-dong Village, $380 \mathrm{~m}$.

Setodes charax Yang \& Morse, 2020

Yang et al. 2020. Zootaxa, 4732 (1): 138-160

Type specimens. Holotype: $\widehat{\partial}$, China, Sichuan, Chong-zhou City, Ji-guan-shan Town, An-zi River Natural Preserve Station, Shao-yao $\operatorname{creek}\left(30.8018^{\circ} \mathrm{N}, 103.2192^{\circ} \mathrm{E}\right.$ alt. $\left.1540 \mathrm{~m}\right)$. Paratypes: $1 \hat{\delta}^{\hat{y}}$, same data as holotype.

Setodes scutatus Yang \& Morse, 2020

Yang et al. 2020. Zootaxa, 4732 (1): 138-160

Type specimens. Holotype: $\widehat{\partial}$, China, Shanxi, Yi-cheng County, Xi-yan-zhen Town, Da-he Villege, Sangou-he

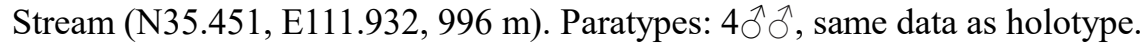

Odontoceridae

Psilotreta brevispinosa Qiu \& Yan, 2020

Qiu \& Yan. 2020. European Journal of Taxonomy, 601: 1-11

Type specimen. Holotype: ${ }^{\lambda}$, China, Henan, Xinyang County, Tian-mu-shan Nature Preserve, near the ranger's

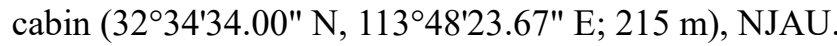

Psilotreta furcata Qiu \& Yan, 2020

Qiu \& Yan. 2020. European Journal of Taxonomy, 601: 1-11

Type specimens. Holotype: $1{ }^{\jmath}$, China, Hubei, Yingshan County, Tao-hua-chong (3059'33.31" N 11559'45.87" E; 469 m), NAU. Paratype: $1{ }^{\lambda}$, same collection data as for holotype, HUST. 
刘童神, 陈静, 姜立云, 乔格侠 (2021) 中国半翅目等29目昆虫2020年新分类单元. 生物多样性, 29, 1050-1057. http://www.biodiversity-science.net/CN/10.17520/biods.2021200.

Phraepsyche acuminata Yang \& Morse, 2020

Yang et al. 2020. Zootaxa, 4732 (1): 138-160

Type specimens. Holotype: $\hat{\jmath}$, China, Guangxi, Tian-lin County, Cen-wang-lao-shan Provincial Forest Preserve, Yang-cun He, tributary of Bu-liu He, trailhead at An-jia-ping, County Road 794 marker $43.2 \mathrm{~km}, \sim 2.5 \mathrm{~km}$ trail, 1155 m. Paratypes: China, Guang-xi: Long-lin County Jin-zhong-shan Provincial Forest Preserve Duo-gui Gou, $\sim 1.5 \mathrm{~km}$ $\mathrm{N}$ of Xi-she village (N24.58245, E104.91415, $1145 \mathrm{~m}$ ), 10. Guang-dong: Longmeng County Nan-kun Shan Provincial Nature Preserve Tian Tang Ding He (N23.64370, E113.84729, 542 m)

Psilotreta longicornis Yang \& Morse, 2020

Yang et al. 2020. Zootaxa, 4732 (1): 138-160

Type specimens. Holotype: ${ }^{\lambda}$, China, ZheJiang, City of Tai Zhou, Lin-hai County, Kuo-cang-shan [Mt.] (N28.83, E120.98, 450 m). Paratype: $1 \partial^{\lambda}$, same data as holotype.

Psychomyia complexa Li, Morse \& Peng, 2020

Psychomyiidae

Peng et al. 2020. Zootaxa, 4860 (2): 227-242

Type specimens. Holotype: $\widehat{\partial}$, China, Hubei, Ma-cheng County, Tong-jian-chong River, $27 \mathrm{~km}$ north of Macheng country $\left(31^{\circ} 23^{\prime} 43.63^{\prime \prime} \mathrm{N}, 115^{\circ} 7^{\prime} 21.77^{\prime \prime} \mathrm{E}, 150 \mathrm{~m}\right)$, NJAU. Paratypes: $1{ }^{\top}$, China, An-hui, Dong-zhi city, Feng-shui village, Qiu-pu-qian river, $11 \mathrm{~km}$ southeast of Dong-zhi city $\left(29^{\circ} 57^{\prime} 44.45^{\prime \prime} \mathrm{N}, 117^{\circ} 1^{\prime} 26.12^{\prime \prime} \mathrm{E}, 30 \mathrm{~m}\right)$, NJAU; China, Hu-bei: $14 \hat{\partial}$, same data as holotype; $6 \hat{\partial}$, Jin-shan-xian $50 \mathrm{~km} \mathrm{N.W}$. of Yincheng Trib. of Da-fu-shui, $90 \mathrm{~m}$, CUAC; China, Jiang-xi: $35 \partial^{\curvearrowright}$, $10 \mathrm{~km}$ S. of Gui-xi city, Xi-qi-he( $\left.31^{\circ} 16^{\prime} 44.40^{\prime \prime} \mathrm{N}, 113^{\circ} 12^{\prime} 12.00^{\prime \prime} \mathrm{E}, 30 \mathrm{~m}\right)$, NJAU.

Psychomyia conoidea Peng, Wang \& Sun, 2020

Peng et al. 2020. Zootaxa, 4860 (2): 227-242

Type specimens. Holotype: ${ }^{\lambda}$, China, Zhejiang, De-qing County, Mo-gan-shan $\left(30^{\circ} 35^{\prime} 15^{\prime \prime} \mathrm{N}, 119^{\circ} 50^{\prime} 2^{\prime \prime} \mathrm{E}, 134 \mathrm{~m}\right)$, NJAU. Paratypes: $4 \partial^{\widehat{\partial}}$, same data as holotype; $1 \partial^{\lambda}$, China, Zhejiang, Lin-an County, Da-shan $\left(30^{\circ} 24^{\prime} 42^{\prime \prime} \mathrm{N}\right.$, $\left.119^{\circ} 36^{\prime} 44^{\prime \prime} \mathrm{E}, 577 \mathrm{~m}\right)$, NJAU.

Psychomyia obtorta Peng, Wang \& Sun, 2020

Peng et al. 2020. Zootaxa, 4860 (2): 227-242

Type specimen. Holotype: ${ }^{\lambda}$, China, Jiang-xi, Long-nan County, Da-qiu-tian Conservation Station of Jiulian-shan National Nature Reserve $\left(24^{\circ} 21^{\prime} 00^{\prime \prime} \mathrm{N}, 114^{\circ} 16^{\prime} 12^{\prime \prime} \mathrm{E}, 377 \mathrm{~m}\right)$, NJAU.

Psychomyia tripetala Peng, Wang \& Sun, 2020

Peng et al. 2020. Zootaxa, 4860 (2): 227-242

Type specimen. Holotype: $\widehat{\partial}$, China, Jiang-xi, Long-nan City, Da-qiu-tian Conservation Station of Jiu-lianshan National Nature Reserve $\left(24^{\circ} 21^{\prime} 00^{\prime \prime} \mathrm{N}, 114^{\circ} 16^{\prime} 12^{\prime \prime} \mathrm{E}, 377 \mathrm{~m}\right)$, NJAU. 
附录 2 本研究所涉及模式标本保藏地点的中英文名称及缩写

Appendix 2 List of abbreviation, Chinese and English names of deposit sites of type specimens in this study

\begin{tabular}{|c|c|c|c|c|c|}
\hline $\begin{array}{l}\text { 发表文献中列出标本存放地名称 } \\
\text { Names of deposit sites in primary literatures }\end{array}$ & $\begin{array}{l}\text { 当前存放单位英文名称 } \\
\text { English name of current deposit sites }\end{array}$ & $\begin{array}{l}\text { 发表文献中列出标本存放地中文名称 } \\
\text { Chinese name of current deposit sites }\end{array}$ & $\begin{array}{l}\text { 名称缩写 } \\
\text { Abbreviation }\end{array}$ & $\begin{array}{l}\text { 存放物种数量 } \\
\text { Number }\end{array}$ & $\begin{array}{l}\text { 属性 } \\
\text { Property }\end{array}$ \\
\hline $\begin{array}{l}\text { College of Plant Protection, Southwest University } \\
\text { Institute of Entomology, Southwest University }\end{array}$ & Southwest University & 西南大学 & SWU & 12 & 高校 \\
\hline Zhongshan Customs Technology Center & Zhongshan Customs Technology Center & 中山海关技术中心 & ZCTC & 2 & 其他 \\
\hline Nantong University & Nantong University & 南通大学 & NU & 1 & 高校 \\
\hline $\begin{array}{l}\text { Department of Entomology, College of Plant Protection, Nanjing } \\
\text { Agricultural University }\end{array}$ & Nanjing Agricultural University & 南京农业大学 & NJAU & 8 & 高校 \\
\hline Shanghai Entomological Museum & $\begin{array}{l}\text { Shanghai Entomological Museum, Chinese Academy of } \\
\text { Sciences }\end{array}$ & 中国科学院上海昆虫博物馆 & SEMCAS & 12 & $\begin{array}{l}\text { 科研院 } \\
\text { 所 }\end{array}$ \\
\hline Shanghai Entomological Museum, Chinese Academy of Sciences & & & & & \\
\hline Senckenberg Museum of Natural History & $\begin{array}{l}\text { Senckenberg Museum of Natural History, Görlitz, } \\
\text { Germany }\end{array}$ & 森根堡自然博物馆 & SMNG & 1 & 博物馆 \\
\hline Northeast Institute of Geography and Agroecology, Chinese & Northeast Institute of Geography and Agroecology, & 中国科学院东北地理与农业生态研究 & IGACAS & 3 & 科研院 \\
\hline Academy of Sciences & Chinese Academy of Sciences & 所 & & & 所 \\
\hline Shanghai Natural History Museum & Shanghai Natural History Museum & 上海自然博物馆 & SNHM & 5 & 博物馆 \\
\hline Universidad Nacional Autónoma de México & $\begin{array}{l}\text { Universidad Nacional Autónoma de México, Mexico } \\
\text { City, Mexico }\end{array}$ & 墨西哥国立自治大学 & UNAM & 1 & 高校 \\
\hline Insect Collection of Jiangsu University of Science and Technology & $\begin{array}{l}\text { Jiangsu University of Science and Technology, Jiangsu, } \\
\text { China }\end{array}$ & 江苏科技大学 & ICJUST & 10 & 高校 \\
\hline Museum of Zoology, Lausanne, Switzerland & Museum of Zoology, Lausanne, Switzerland & 洛桑动物博物馆 & MZL & 1 & 博物馆 \\
\hline Hungarian Natural History Museum, Budapest, Hungary & $\begin{array}{l}\text { Hungarian Natural History Museum, Budapest, } \\
\text { Hungary }\end{array}$ & 匈牙利自然历史博物馆 & HNHM & 6 & 博物馆 \\
\hline
\end{tabular}


刘童神, 陈静, 姜立云, 乔格侠 (2021) 中国半翅目等29目昆虫2020年新分类单元. 生物多样性, 29, 1050-1057. http://www.biodiversity-science.net/CN/10.17520/biods.2021200.

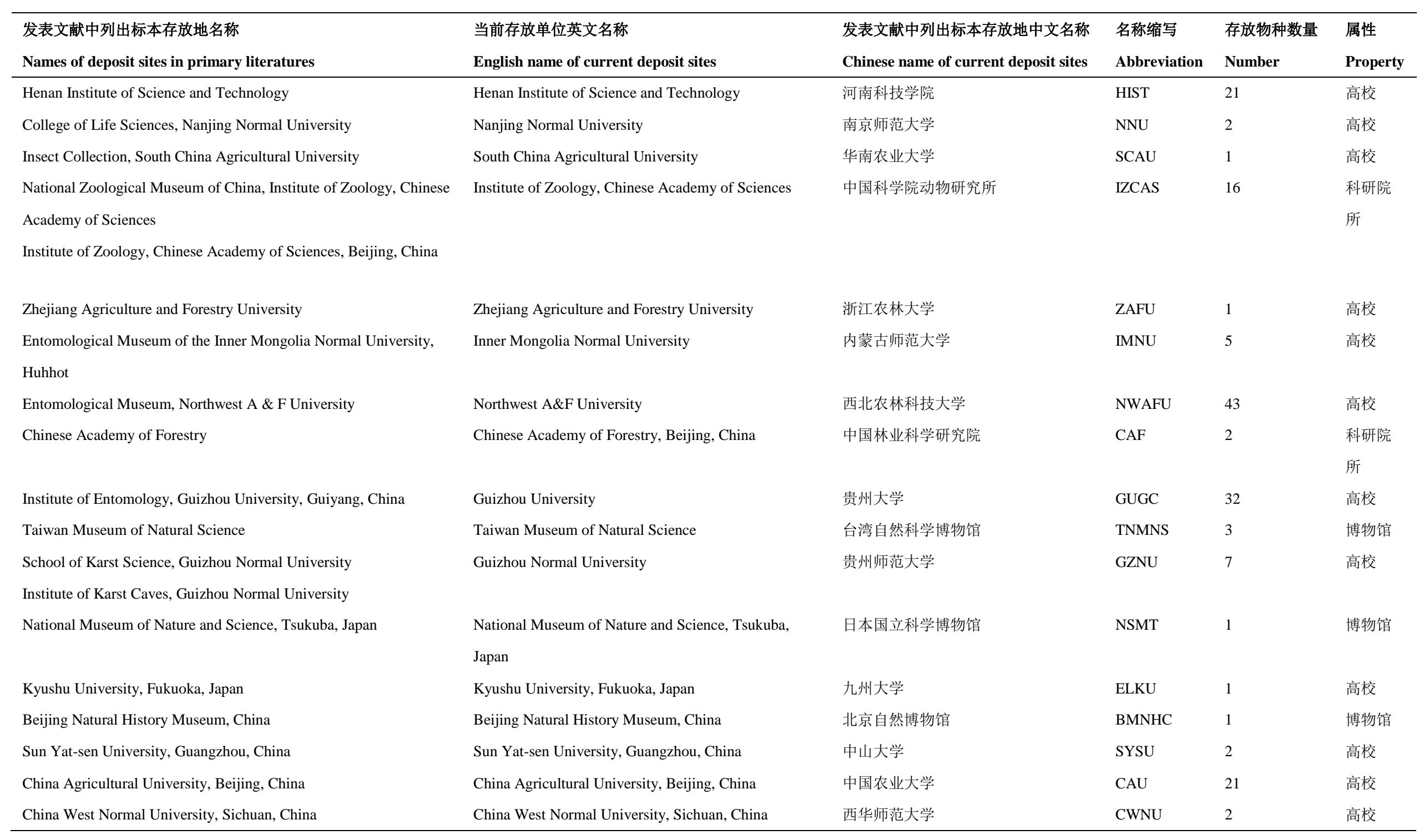


刘童神, 陈静, 姜立云, 乔格侠 (2021) 中国半翅目等29目昆虫2020年新分类单元. 生物多样性, 29, 1050-1057. http://www.biodiversity-science.net/CN/10.17520/biods.2021200.

\begin{tabular}{|c|c|c|c|c|c|}
\hline $\begin{array}{l}\text { 发表文献中列出标本存放地名称 } \\
\text { Names of deposit sites in primary literatures }\end{array}$ & $\begin{array}{l}\text { 当前存放单位英文名称 } \\
\text { English name of current deposit sites }\end{array}$ & $\begin{array}{l}\text { 发表文献中列出标本存放地中文名称 } \\
\text { Chinese name of current deposit sites }\end{array}$ & $\begin{array}{l}\text { 名称缩写 } \\
\text { Abbreviation }\end{array}$ & $\begin{array}{l}\text { 存放物种数量 } \\
\text { Number }\end{array}$ & $\begin{array}{l}\text { 属性 } \\
\text { Property }\end{array}$ \\
\hline Institute of Entomology, College of Life Sciences, Nankai & Nankai University, Tianjin, China & 南开大学 & $\mathrm{NKU}$ & 6 & 高校 \\
\hline \multicolumn{6}{|l|}{ University, Tianjin, China } \\
\hline $\begin{array}{l}\text { Department of Entomology, the National Museum, Prague, Czech } \\
\text { Republic }\end{array}$ & National Museum, Prague, Czech Republic & 布拉格国家博物馆 & NMPCR & 2 & 博物馆 \\
\hline College of Forestry, Jiangxi Agricultural University, Jiangxi, China & Jiangxi Agricultural University & 江西农业大学 & JAU & 1 & 高校 \\
\hline $\begin{array}{l}\text { Department of Forestry Protection, Beijing Forestry University, } \\
\text { Beijing, China }\end{array}$ & Beijing Forestry University, Beijing, China & 北京林业大学 & BFUC & 1 & 高校 \\
\hline $\begin{array}{l}\text { Odonata Collection of the Kunming Institute of Zoology, Chinese } \\
\text { Academy of Sciences, Kunming, Yunnan Province, China }\end{array}$ & $\begin{array}{l}\text { Kunming Institute of Zoology, Chinese Academy of } \\
\text { Sciences, Yunnan, China }\end{array}$ & 中国科学院昆明动物研究所 & KIZCAS & 1 & $\begin{array}{l}\text { 科研院 } \\
\text { 所 }\end{array}$ \\
\hline Naturalis Biodiversity Center, Leiden, Netherlands & Naturalis Biodiversity Center, Leiden, Netherlands & 荷兰自然生物多样性中心 & RMNH & 3 & 其他 \\
\hline \multicolumn{5}{|l|}{ College of Life Sciences, Hebei University, Baoding, China } & 高校 \\
\hline $\begin{array}{l}\text { Northwest Plateau Institute of Biology, Chinese Academy of } \\
\text { Sciences, Xining, China }\end{array}$ & $\begin{array}{l}\text { Northwest Plateau Institute of Biology, Chinese } \\
\text { Academy of Sciences, Xining, China }\end{array}$ & 中国科学院西北高原生物研究所 & NPIBCAS & 1 & $\begin{array}{l}\text { 科研院 } \\
\text { 所 }\end{array}$ \\
\hline $\begin{array}{l}\text { Institute of Entomoceutics Research, Dali University } \\
\text { Biological Science Museum, Dali University }\end{array}$ & \multicolumn{4}{|c|}{ Biological Science Museum, Dali University } & 高校 \\
\hline \multicolumn{5}{|l|}{ China } & 高校 \\
\hline Museum of Biology, East China Normal University & East China Normal University, Shanghai, China & 华东师范大学 & ECNU & 7 & 高校 \\
\hline College of Plant Protection, Shandong Agricultural University & Shandong Agricultural University, Shandong, China & 山东农业大学 & SAU & 1 & 高校 \\
\hline \multicolumn{6}{|l|}{ University } \\
\hline Entomological Museum of Hechi University, Yizhou, China & Hechi University, Yizhou, China & 河池学院 & EMHU & 8 & 高校 \\
\hline Natural History Museum, London, United Kingdom & Natural History Museum, London, United Kingdom & 伦敦自然历史博物馆 & NHML & 8 & 博物馆 \\
\hline
\end{tabular}


刘童神, 陈静, 姜立云, 乔格侠 (2021) 中国半翅目等29目昆虫2020年新分类单元. 生物多样性, 29, 1050-1057. http://www.biodiversity-science.net/CN/10.17520/biods.2021200.

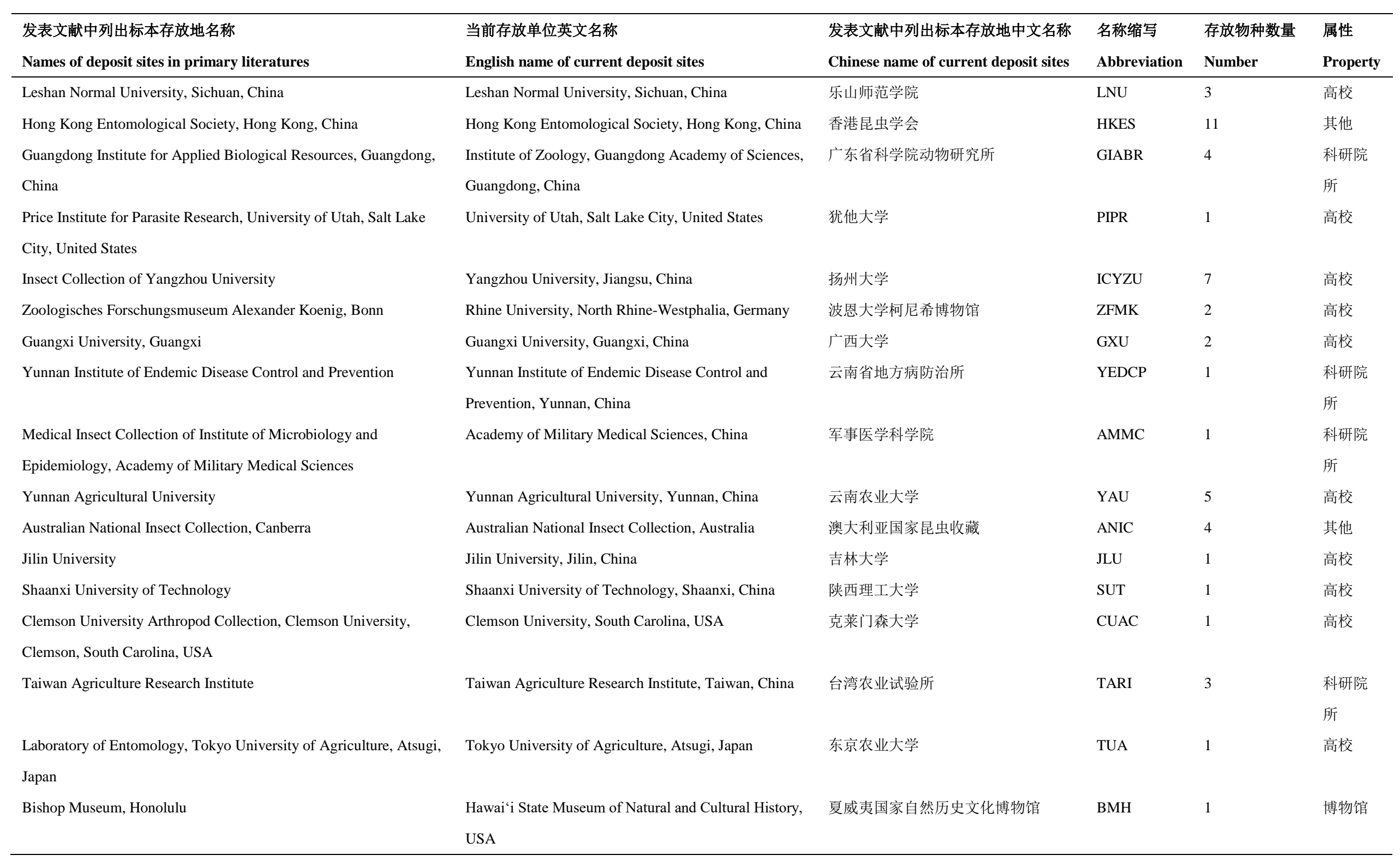


刘童祎, 陈静, 姜立云, 乔格侠 (2021) 中国半翅目等29目昆虫2020年新分类单元. 生物多样性, 29, 1050-1057. http://www.biodiversity-science.net/CN/10.17520/biods.2021200.

\begin{tabular}{|c|c|c|c|c|c|}
\hline 发表文献中列出标本存放地名称 & 当前存放单位英文名称 & 发表文献中列出标本存放地中文名称 & 名称缩写 & 存放物种数量 & 属性 \\
\hline Names of deposit sites in primary literatures & English name of current deposit sites & Chinese name of current deposit sites & Abbreviation & Number & Property \\
\hline National Chung Hsing University, Taiwan, China & National Chung Hsing University, Taiwan, China & 中兴大学 & $\mathrm{NCHU}$ & 2 & 高校 \\
\hline Naturhistorisches Museum, Basel, Switzerland & Naturhistorisches Museum, Basel, Switzerland & 巴塞尔自然史博物馆 & NHMB & 2 & 博物馆 \\
\hline Moscow State Pedagogical University & Moscow State Pedagogical University & 俄罗斯莫斯科国立师范大学 & MSPU & 1 & 高校 \\
\hline \multirow[t]{2}{*}{ Guizhou Institute of Biology } & Guizhou Institute of Biology, Guiyang, China & 贵州科学院生物研究所 & GZIB & 8 & 科研院 \\
\hline & & & & & 所 \\
\hline Collection Ernst Heiss at the Tiroler Landesmuseum Innsbruck & & Ernst Heiss 个人收藏 & CEHI & 2 & 个人 \\
\hline Personal collection of Chao Wu (PCW) & & Chao Wu 个人收藏 & PCW & 2 & 个人 \\
\hline Personal collection of Jiazhi Zhang, Shanghai, China & & Jiazhi Zhang 个人收藏 & $\mathrm{CJZ}$ & 1 & 个人 \\
\hline Personal collection of Hao-miao Zhang & & Hao-miao Zhang 个人收藏 & PHMZ & 1 & 个人 \\
\hline
\end{tabular}

